\title{
Subsequential scaling limits for Liouville graph distance
}

\author{
Jian Ding* \\ University of Pennsylvania \\ Alexander Dunlap ${ }^{\dagger}$ \\ Stanford University
}

October 4, 2019

\begin{abstract}
For $0<\gamma<2$ and $\delta>0$, we consider the Liouville graph distance, which is the minimal number of Euclidean balls of $\gamma$-Liouville quantum gravity measure at most $\delta$ whose union contains a continuous path between two endpoints. In this paper, we show that the renormalized distance is tight and thus has subsequential scaling limits at $\delta \rightarrow 0$. In particular, we show that for all $\delta>0$ the diameter with respect to the Liouville graph distance has the same order as the typical distance between two endpoints.
\end{abstract}

\section{Introduction}

Let $\mathbb{R}$ be a rectangular subset of $\mathbf{R}^{2}$ (the Euclidean plane), and let $\mathbb{R}^{*}$ be a box centered around $\mathbb{R}$ whose sides are separated from those of $\mathbb{R}$ by twice the Euclidean diameter of $\mathbb{R}$. Let $h_{\mathbb{R}^{*}}$ be a Gaussian free field with Dirichlet boundary conditions on $\mathbb{R}^{*}$ and let $\mu_{\mathbb{R}}$ be the Liouville quantum gravity (LQG) measure on $\mathbb{R}$, at inverse temperature $\gamma \in(0,2)$, induced by $h_{\mathbb{R}^{*}}$. (We precisely define these objects in Section 2; see also the surveys $[40,5,34]$.) In this paper, we consider the Liouville graph distance with parameter $\delta$ on $\mathbb{R}$, which for any two points $x, y \in \mathbb{R}$ is given by $\underline{d}_{\mathbb{R}} \delta(x, y)$, the minimal number of Euclidean balls of LQG measure at most $\delta$ that it takes to cover a path between $x$ and $y$. (Again, see Section 2 for a precise definition.) Let $Q_{\delta}$ be the median Liouville graph distance from the left to the right side of $\mathbb{R}$. Our goal in this paper is to prove the following:

Theorem 1.1. For any sequence $\delta_{n} \downarrow 0$, there is a subsequence $\left(\delta_{n_{k}}\right)$ and a limiting metric $\underline{d}_{\mathbb{R}}$ so that

$$
Q_{\delta_{n_{k}}}^{-1} \underline{d}_{\mathbb{R} \delta_{n_{k}}} \rightarrow \underline{d}_{\mathbb{R}}
$$

in distribution with respect to the uniform topology on functions $\mathbb{R} \times \mathbb{R} \rightarrow \mathbf{R}$. Moreover, $\underline{d}_{\mathbb{R}}$ is Höldercontinuous with respect to the Euclidean metric on $\mathbb{R}$, and the Euclidean metric on $\mathbb{R}$ is Hölder-continuous with respect to $\underline{d}_{\mathbb{R}}$.

A consequence of Theorem 1.1, but also a key step in its proof (see Proposition 6.4 below), is that for any $\delta$, the LQG diameter of a box is comparable to its left-right crossing distance:

Theorem 1.2. For any $\varepsilon>0$, we have a $C=C(\varepsilon)$ so that, for all $\delta>0$,

$$
\mathbf{P}\left(\max _{x, y \in \mathbb{R}} \underline{d}_{\mathbb{R}, \delta}(x, y) \geq C(\varepsilon) Q_{\delta}\right) \leq \varepsilon .
$$

*Partially supported by National Science Foundation (NSF) grant DMS-1757479 and an Alfred Sloan fellowship.

${ }^{\dagger}$ Partially supported by a National Science Foundation Graduate Research Fellowship under grant DGE-1147470. 
The normalizing constant $Q_{\delta}$ is poorly-understood, and the arguments in this paper do not rely on precise estimates of its value. However, we will show the following in the course of the proof of Theorem 1.1:

Theorem 1.3. We have a constant $0<C<\infty$ so that for all $\delta$ we have

$$
C^{-1} \delta^{-C^{-1}} \leq Q_{\delta} \leq C \delta^{-C}
$$

\subsection{Background and motivation}

In the seminal paper [21], the Gaussian multiplicative chaos was defined and constructed as a random measure obtained by exponentiating log-correlated Gaussian fields. The last decade has seen extensive study of Gaussian multiplicative chaos as well as Liouville quantum gravity, ${ }^{1}$ which is an important special case of Gaussian multiplicative chaos where the underlying log-correlated field is a two-dimensional Gaussian free field. See for example [21, 17, 33, 34, 6, 35, 4].

Recently, a huge amount of effort has been devoted to understanding the random metric associated with LQG. Building on [30, 16], in [31, 28, 29] the authors constructed in the continuum a random metric which is presumably the scaling limit of the LQG distance for the specific choice $\gamma=\sqrt{8 / 3}$, and proved deep connections with the Brownian map[22, 23, 27]. Note that in these works there is no mention of a discrete approximation of the metric. In [14, 9, 20, 19, 10], various bounds on the distance and properties of the geodesics were obtained. In $[13,11]$, some non-universality aspects (when considering underlying logcorrelated fields other than GFF) for LQG distances were demonstrated. In [26, 12], a type of equivalence between the Liouville graph distance and the heat kernel for Liouville Brownian motion was proved. In [10], it was shown that there is a single number which determines the distance exponents for a few reasonable choices of distances associated with LQG, as well as the distance exponents for random planar maps.

However, despite much dedicated effort, the two most outstanding problems related to LQG distances remain open: (1) to compute the exact distance exponents for LQG distances (although this is now known for $\gamma=\sqrt{8 / 3}$ by $[3,10])$, and (2) to derive a scaling limit of natural discrete approximations of LQG distances. The present paper makes some progress towards understanding the scaling limits of LQG distances.

\subsection{Two closely-related works}

The present article is closely related to [8, 15]. In [8] it was shown that discrete Liouville first-passage percolation (shortest-path metric where the vertices are weighted by the exponential of the discrete GFF) has subsequential scaling limits for sufficiently small $\gamma>0$. On the other hand, in [15], the authors considered the case when the underlying field is a type of log-correlated Gaussian field (in the continuum) with short-range correlations (a so-called $\star$-scale invariant field) and showed that there exists a parameter $\gamma^{*}>0$ such that the corresponding Liouville first-passage percolation has a subsequential scaling limit for all $\gamma<\min \left(\gamma^{*}, 0.4\right)$. The main contribution of the present article is that the result for Liouville graph distance is valid throughout the subcritical regime; i.e. $\gamma \in(0,2)$. A few further remarks are in order:

- In both [8] and [15], the authors worked with Liouville first-passage percolation, while in the present article we work with Liouville graph distance. They are both natural approximations of LQG distances and at the moment we are equally satisfied with proving results for either of these choices (or any other reasonable choices). As noted earlier, in [10], universality of the dimension exponents was proved for all reasonable choices of metric we know when we stick to the GFF as the underlying field (but we should also note that the dimension exponent is not expected to be universal between GFF and $\star$-scale invariant field; see [14]. Here we choose to work with Liouville graph distance because it resembles

\footnotetext{
${ }^{1}$ Our terminology for Liouville quantum gravity follows that in [17]. This is somewhat different from that adopted in Liouville field theory, and one should be cautious about the underlying mathematical meaning of LQG in Liouville field theory.
} 


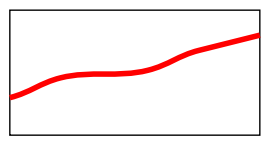

(a) Easy crossing.

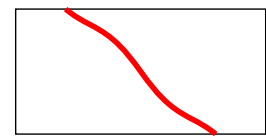

(b) Hard crossing.

Figure 1.1: Easy and hard crossings.

random planar maps in a more obvious fashion, and because it is (slightly) more directly connected to the heat kernel for Liouville Brownian motion [12].

- In both $[8,15]$, in the regime where subsequential scaling limits were proved, a crucial step of the proofs was that the box diameter has the same order as the typical distance between two points. We also note that in [10], it was shown for all $\gamma \in(0,2)$ that the exponents for the diameter and the typical distance are the same. The present article similarly shows that in our setting, the diameter of a box has the same order as the typical distance between two points.

- In [15], results regarding the Weyl scaling were also obtained. Weyl scaling is considered neither in [8] nor in the present article.

- The $\star$-scale invariant fields form an important class of log-correlated Gaussian fields, and they have been studied previously in $[2,25]$ for instance. We note that the Gaussian free field is not a $\star$-scale invariant field.

- At the moment, we are equally satisfied with proving results when the underlying fields are discrete or continuous. But the continuous case appears to be simpler thanks to conformal invariance-based arguments introduced in [15].

We now discuss similarities and differences in proof methods. In [8], we presented a framework combining multiscale analysis and Efron-Stein-type arguments with Russo-Seymour-Welsh (RSW)-type estimates (originally introduced in $[36,38,37]$ ), which relate distances between boundaries of rectangles in easy and hard directions. (Here and throughout the paper, the "easy" direction across a rectangle refers to crossings between the two longer sides, while the "hard" direction refers to crossings between the two shorter sides; see Figure 1.1.) This is more or less the framework used both in [15] and in the present article.

The key difference between [8] and [15] lies in the implementation of the RSW estimate. In [8] the RSW estimate was inspired by [41], while in [15] it was proved using approximate conformal invariance. The proof in [8] draws a natural connection between random distances and percolation theory. It also presents a more widely-applicable framework — based on the method of [41] - for proving RSW estimates, as it does not rely on conformal invariance (and thus works for instance in the lattice case, or potentially for more general fields). However, while the proof in [41] (for Voronoi percolation) is simply beautiful, much of the beauty was obscured when it was implemented in [8], due to the severe complications involved in considering lengths of paths rather than simply connectivity. By contrast, the proof method of [15], taking advantage of conformal invariance, is insightful and beautiful. While the method of [15] does suffer the drawback of relying on conformal invariance and thus may not be as widely applicable as the method in [8], this drawback is irrelevant here since at the moment we are satisfied with the case of a conformallyinvariant field. For the present article, while it seems likely that an RSW proof following [8] is possible (although it would require improving the analysis in [8] in a number of places), we switch to a conformal invariance-based proof as in [15] for the sake of simplicity.

In [8], since $\gamma$ is very small, in the multiscale analysis the influence from the coarse field (which one can roughly understand as circle averages with respect to macroscopic circles) is negligible, which simplifies 
the analysis in a number of ways. (The situation is in some sense similar in [15] as there it is only shown that $\gamma^{*}>0$.) Thus, the main new technical challenge in the present article is controlling the influence from coarse field for $0<\gamma<2$. (Naturally, it is most difficult when $\gamma$ is close to 2.) One manifestation of this challenge is the fact that LQG measure only has a finite $p$ th moment for $p$ approaching 1 as $\gamma$ approaches 2 . While this creates challenges in the multiscale analysis, from a conceptual point of view our proof crucially relies on the fact that the LQG measures for all Euclidean balls with radius at most $\varepsilon$ uniformly converge to 0 for $\gamma \in(0,2)$ - this fact is essential crucial for the diameter to have the same order as the typical distance.

\subsection{Ingredients of the proof}

Here we describe the essential structure of our proof.

By a chaining argument similar to the ones used in [8, 15], we can bound the diameter of a box by a sum of hard crossing distances of dyadic subboxes at successively smaller scales. (See Proposition 6.4.) Controlling the fluctuation of box diameters uniformly in the scale is essentially enough to uniformly control the fluctuations of a Hölder norm of the metric with respect to the Euclidean metric, which yields tightness in the uniform norm. Thus, our essential goal is to bound the fluctuations of hard crossing distances, again uniformly in the scale. The form of our bound on these fluctuations will be a variance bound on the logarithm of these fluctuations (Theorem 5.1), which allows us to relate different quantiles of the scale as described in Lemma A.1.

Our bound on the variance of the logarithm of the hard crossing distance takes place in the framework of the Efron-Stein inequality. We write the underlying Gaussian free field as a function of the space-time white noise, partition space-time into chunks, and then express the variance of the logarithm of the Liouville graph distance as the sum of the expected squared multiplicative (since we are considering the logarithm) changes in the distance when each box is resampled. The white noise decomposition is by now a widely-used way to decompose the GFF into a "fine field," with no regularity but only short-range correlations, and a "coarse field," which is smooth but has long-range correlations. See for example [34,39] for general descriptions of the white noise decomposition and $[9,12]$ for previous uses of this decomposition in the setting of the LQG metric.

We control the fluctuations due to the coarse field using a standard Gaussian concentration argument in Subsection 5.3.1; this is sufficient because we choose the decomposition so that the coarse field is sufficiently smooth. The more serious task is to control the fluctuations due to the fine field. Of course, the most pronounced effect of the fine field in a certain box is on the part of the geodesic close to the same box. Controlling the fluctuations on this part of the geodesic (done in Subsection 5.3.3) essentially has two key components:

- First, we observe that since the coarse field is smooth, and thus can be treated as a constant on the small scale box, it suffices to control the change in the distance for the LQG metric in boxes at a smaller scale. This smaller scale, though, is not the size of the smaller boxes. From the perspective of the Liouville graph distance, when the coarse field is positive, subboxes look larger than they are, while when the coarse field is negative, subboxes look smaller than they are. This is to say that the small boxes have an "effective size" depending on the value of the coarse field. (This is quantified by the scaling covariance of the Liouville quantum gravity; see Proposition 2.13 and Proposition 2.38.) The requirement that $\gamma<2$ is precisely what is needed to ensure that, even considering the maximum effect of the coarse field, the effective size of the small boxes is strictly smaller than that of the overall large box with high probability.

- Second, the fact that the subboxes have a smaller effective size with high probability means that we can apply an inductive procedure. We assume that we have already proved our variance bound at smaller scales, and thus can use this variance bound to bound quantiles of crossing distances in our 
small boxes. This works because the variance bound, together with the RSW result (Theorem 3.4) and percolation-type arguments (given in Section 4), allows us to show that crossing distances are concentrated, and that "easy" and "hard" crossing distances are multiplicatively comparable. This means that, using the inductive hypothesis, we can replace the part of the geodesic close to the resampled box with a geodesic around a surrounding annulus while only increasing the distance by, typically, a constant factor. Moreover, since again the effective size of the smaller boxes is smaller than the size of the large box, the increase in the distance incurred in this way is also small compared to the total crossing distance of the large box. (Here, we need to know that typical distances increase at least polynomially in the scale, which is established in Proposition 4.4.) This is what is needed to bound the variance of the logarithm.

As we have noted, the improvement of this paper compared to [8, 15] is that we are able to obtain the tightness result for all $\gamma \in(0,2)$. The main ingredient for this, not used in [8, 15], is the scaling covariance of the Liouville quantum gravity described above. This allows us to perform a more precise multiscale analysis than is done in either of these preceding works. In particular, this works because we are able to show that precisely when $\gamma<2$, subboxes of a larger box have a a strictly smaller "effective size" than the larger box with high probability.

\subsection{Organization of the paper}

In Section 2 we introduce our cast of characters, collecting a number of (semi-)standard facts about Gaussian free fields, Liouville quantum gravity, and Liouville graph distance. In Section 3 we prove a conformal covariance-based RSW estimate for Liouville quantum gravity, in the spirit of [15]. In Section 4 we prove concentration bounds on crossing distances using percolation arguments in a multi-scale analysis framework, and set up some quantities regarding the relationship between different quantiles of crossing distances, which will form the objects of our inductive procedure. (We defer the introduction of these quantities until that point because they depend on certain constants which are introduced in the lemmas of Section 3 and Section 4.) In Section 5, we carry out the Efron-Stein argument, and in Section 6 we show that the diameter is within a constant of the typical distance between two points, via a chaining argument, on our way to proving the tightness of the metrics which is the main ingredient in Theorems 1.1, 1.2, and 1.3. In Section 7, we prove that the the Euclidean metric is Hölder-continuous with respect to any limiting metric, completing the proof of Theorem 1.1. Finally, we have relegated several technical lemmas in analysis and probability, that do not relate in particular to the subjects of this paper, to an appendix (beginning on page 60).

\subsection{Acknowledgments}

We thank Ewain Gwynne, Subhajit Goswami, Nina Holden, Jason Miller, Dat Nguyen, Josh Pfeffer, and Xin Sun for interesting conversations. We especially thank Hugo Falconet for pointing out an error in an earlier version of the manuscript. We are also grateful to an anonymous referee for an extremely careful reading and many insightful comments, which greatly helped to improve the exposition.

\section{Preliminaries}

\subsection{Notation}

We denote by $\mathbf{R}$ the set of real numbers and by $\mathbf{Q}$ the set of rational numbers. If $x, y \in \mathbf{R}$, we will use the notation $x \wedge y=\min \{x, y\}, x \vee y=\max \{x, y\}, x^{+}=x \vee 0$, and $x^{-}=x \wedge 0$. If $x \in \mathbf{R}^{2}$, we will denote by $|x|$ the Euclidean norm of $x$. We denote by $B(x, r)$ the Euclidean open ball with center $x$ and radius $r$. We will often 
use blackboard-bold letters to refer to subsets of $\mathbf{R}^{2}$. If $\mathbb{X} \subset \mathbf{R}^{2}$, then let $\operatorname{diam}_{E}(\mathbb{X})$ denote the Euclidean diameter of $\mathbb{X}$, and let $\overline{\mathbb{X}}$ denote the closure of $\mathbb{X}$ in the Euclidean topology.

By a domain we mean an open connected subset of $\mathbf{R}^{2}$. By a box we will mean a closed rectangular subset of $\mathbf{R}^{2}$ whose axes are aligned with the coordinate axes. If $\mathbb{B}$ is a box, let width $(\mathbb{B})$ and height $(\mathbb{B})$ denote the width and height of $\mathbb{B}$, respectively. We define the aspect ratio of $\mathbb{B}$ as

$$
\operatorname{AR}(\mathbb{B})=\frac{\operatorname{width}(\mathbb{B})}{\operatorname{height}(\mathbb{B})}
$$

For a box $\mathbb{B}$ and a scalar $\lambda>0$, define $\lambda \mathbb{B}$ to be the box with the same center as $\mathbb{B}$ but $\lambda$ times the side length.

We will frequently use the notation

$$
\mathbb{B}\left(S_{1}, S_{2}\right)=\left[-S_{1} / 2, S_{1} / 2\right] \times\left[-S_{2} / 2, S_{2} / 2\right]
$$

and

$$
\mathbb{B}(S)=\mathbb{B}(S, 2 S) .
$$

Given a box $\mathbb{B}$, let $\mathrm{L}_{\mathbb{B}}, \mathrm{R}_{\mathbb{B}}, \mathrm{T}_{\mathbb{B}}$, and $\mathrm{B}_{\mathbb{B}}$ denote the left, right, top, and bottom sides of $\mathbb{B}$, respectively. We will often omit the subscript $\mathbb{B}$ if it is clear from context.

If $\mathbb{B} \subset \mathbf{R}^{2}$, we will use the notation

$$
\mathbb{B}^{(R)}=\left\{x \in \mathbf{R}^{2} \mid \operatorname{dist}_{\mathrm{E}}(x, \mathbb{B})<R\right\}=\bigcup_{x \in \mathbb{B}} B(x, R) .
$$

If $\mathbb{B} \subset \mathbf{R}^{2}$ is a box, define $\mathbb{B}^{\circ}$ to be the smallest box containing $\mathbb{B}^{\left(\text {diam }_{E} \mathbb{B}\right)}$, and $\mathbb{B}^{*}$ to be the smallest box containing $\mathbb{B}^{\left(2 \operatorname{diam}_{E} \mathbb{B}\right)}$.

Throughout this paper, we will often work with constants, usually denoted by $C$ or similar notation if they are to be thought of as large, or by $c$ or similar notation if they are to be thought of as small. We will always allow constants to change from line to line in a computation.

\subsection{The Gaussian free field}

If $\mathbb{A} \subset \mathbf{R}^{2}$ is a domain, we will denote by $h_{\mathbb{A}}$ a Gaussian free field with Dirichlet boundary conditions on $\mathbb{A}$. (See $[40,5]$ for more systematic introductions to the GFF.) We recall that the GFF is a distribution-valued stochastic process with covariance function

$$
G^{\mathbb{A}}(x, y)=\pi \int_{0}^{\infty} p_{t}^{\mathbb{A}}(x, y) \mathrm{d} t,
$$

where $G^{\mathbb{A}}$ is the Green's function for the Dirichlet problem on $\mathbb{A}$, and $p_{t}^{\mathbb{A}}$ is the heat kernel for a standard Brownian motion killed on $\partial \mathbb{A}$. If $\mathbb{B} \supset \mathbb{A}$, then we define $h_{\mathbb{B}: \mathbb{A}}$ to be the harmonic interpolation of $h_{\mathbb{B}}$ on $\mathbb{A}$, and simply $h_{\mathbb{B}}$ outside of $\mathbb{A}$. (It is not quite obvious that this harmonic interpolation makes sense, because $h_{\mathbb{B}}$ is a distribution and not a function. See [40] or [5] for the precise construction.) We then have the following standard property. (Again see [40,5].)

Proposition 2.1 (Gibbs-Markov property). The field

$$
h_{\mathbb{B}}=h_{\mathbb{A}}-h_{\mathbb{A}: \mathbb{B}}
$$

is a standard Gaussian free field on $\mathbb{B}$. Moreover, the fields $h_{\mathbb{B}}$ and $h_{\mathbb{A}: \mathbb{B}}$ are independent. 
This paper will rely in crucial ways on multiscale analysis, which means that we will want to consider Gaussian free fields defined on all boxes simultaneously - in other words, we want to couple the Gaussian free fields on all boxes. We can do this by considering a whole-plane GFF $h_{\mathbf{R}^{2}}$ (see [5, Section 4.1]), pinned in some arbitrary way. Then we can define $h_{\mathbb{B}}=h_{\mathbf{R}^{2}}-h_{\mathbf{R}^{2}: \mathbb{B}}$ for each domain $\mathbb{B}$. Alternatively, we can use the construction in [8, Section 2.2.1]: the coupling between GFFs on different domains is easy to define if all domains $\mathbb{B}$ are subsets of some universal box $\mathbb{A}$, because we can define $h_{\mathbb{B}}$ for all $\mathbb{B} \subset \mathbb{A}$ by (2.2). But then the universal box $\mathbb{A}$ can be taken to be arbitrarily large, and it is easy to check that the joint laws of the Gaussian free field on finitely many domains remain the same no matter how large we take $\mathbb{A}$. Thus, by Kolmogorov's extension theorem, we can take $h_{\mathbb{B}}$ to be defined simultaneously on all domains $\mathbb{B} \subset \mathbf{R}^{2}$ so that (2.2) holds for any nested pair of domains.

The following locality property of the Gaussian free field (which can actually be seen as a consequence of the Gibbs-Markov property) will also be essential.

Proposition 2.2. If $\mathbb{A}$ and $\mathbb{A}^{\prime}$ are disjoint domains, then $h_{\mathbb{A}}$ and $h_{\mathbb{A}^{\prime}}$ are independent.

A final crucial property of the Gaussian free field is its conformal invariance. (See [5, Theorem 1.19].)

Proposition 2.3. Suppose that $F: \mathbb{V} \rightarrow \mathbb{V}^{\prime}$ is a conformal map between two domains $\mathbb{V}$ and $\mathbb{V}^{\prime}$. Then $h_{\mathbb{V}^{\prime}}$ has the same law as $h_{\mathbb{V}^{\prime}} \circ F^{-1}$.

\subsection{Gaussian free field estimates}

In this section we introduce certain estimates on the Gaussian free field that will be important throughout the paper.

Lemma 2.4. There is a constant $C<\infty$ so that, if $\mathbb{B}$ is a box with width $(\mathbb{B})$, height $(\mathbb{B}) \in[1 / 3,3]$, and $x, y \in \mathbb{B}$, then

$$
\left|G^{\mathbb{B}^{*}}(x, y)+\log \right| x-y|| \leq C .
$$

Proof. This can be computed from the explicit formula for the Green's function of the Laplacian on the unit disk, using a uniform bound on the restriction of the derivative to $\mathbb{B}$ of a Riemann map from $\mathbb{B}^{*}$ to the unit disk.

Lemma 2.5. There is a constant $C<\infty$ so that the following holds. Let $\mathbb{B} \subset \mathbb{A}$ be two nested boxes such that $\operatorname{AR}(\mathbb{A}), \operatorname{AR}(\mathbb{B}) \in[1 / 3,3]$ and let $x$ be the center point of $\mathbb{B}$. Then we have

$$
\operatorname{Var}\left(h_{\mathbb{A}^{*}: \mathbb{B}^{*}}(x)\right) \leq-\log \frac{\operatorname{diam}_{\mathrm{E}}(\mathbb{B})}{\operatorname{diam}_{\mathrm{E}}(\mathbb{A})}+C .
$$

Proof. This follows simply from writing down the integral expression for the variance of $h_{\mathbb{A}^{*}: \mathbb{B}^{*}}(x)$ and applying (2.3).

Lemma 2.6. There is a constant $C<\infty$ so that if $\mathbb{B} \subset \mathbb{B}^{*} \subset \mathbb{A}$ are boxes with aspect ratios between $1 / 3$ and 3 , then for all $x, y \in \mathbb{B}^{\circ}$, we have

$$
\operatorname{Var}\left(h_{\mathbb{A}: \mathbb{B}^{*}}(x)-h_{\mathbb{A}: \mathbb{B}^{*}}(y)\right) \leq C \frac{|x-y|}{\operatorname{diam}_{\mathrm{E}}(\mathbb{B})} .
$$

The proof of Lemma 2.6 is given in the case when $\mathbb{B}$ is a ball in [9, Lemma 6.1]; the proof is essentially the same in our setting. 
Proposition 2.7. For each $Q, \iota>0$, there is a constant $C=C(Q, \iota)<\infty$ so that the following holds. Let $\mathbb{B}$ be a box so that $\mathrm{AR}(\mathbb{B}) \in[1 / 3,3]$. Let $K \in(1, \infty)$ and let $\mathbb{C}_{1}, \ldots, \mathbb{C}_{J}$, with $J \leq Q K^{2}$, be a set of subboxes of $\mathbb{B}$ such that, for each $1 \leq j \leq J$, we have $\operatorname{AR}\left(\mathbb{C}_{j}\right) \in[1 / 3,3]$ and

$$
\frac{\operatorname{diam}_{\mathrm{E}}(\mathbb{B})}{\operatorname{diam}_{\mathrm{E}}\left(\mathbb{C}_{j}\right)} \in[K / 3,3 K]
$$

Then for all $\theta>0$, for each $j \in\{1, \ldots, J\}$ we have,

$$
\mathbf{P}\left(\max _{x \in \mathbb{C}_{j}^{\circ}} h_{\mathbb{B}^{*}: \mathbb{C}_{j}^{*}}(x) \geq \theta \log K\right) \leq C K^{-\theta^{2} / 2}
$$

and moreover

$$
\mathbf{P}\left(\max _{j=1}^{J} \max _{x \in \mathbb{C}_{j}^{\circ}} h_{\mathbb{B}^{*}: \mathbb{C}_{j}^{*}}(x) \geq \theta \log K\right) \leq C K^{2-\theta^{2} / 2} .
$$

Proof. First we note that by Fernique's inequality ([18], see e.g. [1, Theorem 4.1] and [24, equation (7.4), Theorem 7.1]) and Lemma 2.6, we have a constant $C$ independent of $j$ so that

$$
\mathbf{E}\left(\max _{x \in \mathbb{C}_{j}^{\circ}} h_{\mathbb{B}^{*}: \mathbb{C}_{j}^{*}}(x)\right) \leq C .
$$

Also, by the Borell-TIS inequality (see e.g. [24, Theorem 7.1], [7, Theorem 6.1], or [1, Theorem 2.1]) and Lemma 2.5, we have constant $C$, again independent of $j$, so that

$$
\mathbf{P}\left(\left|\max _{x \in \mathbb{C}_{j}^{\circ}} h_{\mathbb{B}^{*}: \mathbb{C}_{j}^{*}}(x)-\mathbf{E} \max _{x \in \mathbb{C}_{j}^{\circ}} h_{\mathbb{B}^{*}: \mathbb{C}_{j}^{*}}(x)\right| \geq \theta \log K\right) \leq \exp \left\{-\frac{1}{2} \frac{\theta^{2}(\log K)^{2}}{\log K+C}\right\} \leq C K^{-\theta^{2} / 2},
$$

where the constant $C$ has changed from the second to the third expression. Combining (2.7) and (2.8) yields (2.5). Then taking a union bound over $j \in\{1, \ldots, J\}$ yields (2.6).

The following corollary will be used over and over again throughout the paper. It tells us that, for any $\theta_{0}>2$, if we divide a box into order $K^{2}$-many smaller boxes of $1 / K$ times the side length, the maximum "coarse field" imposed on the small boxes will be of size at most $\theta_{0} \log K$ with high probability.

Corollary 2.8. If $\theta_{0}>2$ and $Q, K>1$ are such that $J \leq Q K^{2}$, then there is a constant $C$ depending on $Q$ and $\theta_{0}$ so that the following holds. Suppose that $\mathbb{B}$ is a box so that $\mathrm{AR}(\mathbb{B}) \in[1 / 3,3]$. Let $\mathbb{C}_{1}, \ldots, \mathbb{C}_{J}$ be a set of subboxes of $\mathbb{B}$ so that $\mathrm{AR}\left(\mathbb{C}_{j}\right) \in[1 / 3,3]$ for each $1 \leq j \leq J$ and that

$$
\frac{\operatorname{diam}_{\mathrm{E}}(\mathbb{B})}{\operatorname{diam}_{\mathrm{E}}\left(\mathbb{C}_{j}\right)} \in[K / 3,3 K]
$$

Let

$$
F=\max _{j=1}^{J} \max _{x \in \mathbb{C}_{j}} h_{\mathbb{B}^{*}: \mathbb{C}_{j}^{*}}(x)
$$

Then, for all $v>1$, we have

$$
\mathbf{P}\left[\mathrm{e}^{\gamma F-\gamma \theta_{0} \log K} \geq v\right] \leq C v^{-2 / \gamma} \mathrm{e}^{-\frac{(\log v)^{2}}{2 \gamma^{2} \log K}}
$$


Proof. By (2.6) of Proposition 2.7, we have, for $\theta>0$,

$$
\mathbf{P}\left[F \geq\left(\theta_{0}+\theta\right) \log K\right] \leq C K^{2-\left(\theta_{0}+\theta\right)^{2} / 2} \leq C K^{-2 \theta-\theta^{2} / 2},
$$

with the last inequality because $\theta_{0}>2$. This is equivalent to

$$
\mathbf{P}\left[\mathrm{e}^{\gamma F-\gamma \theta_{0} \log K} \geq K^{\gamma \theta}\right] \leq C K^{-2 \theta-\theta^{2} / 2},
$$

so, putting $\theta=\frac{\log v}{\gamma \log K}$, we have

$$
\mathbf{P}\left[\mathrm{e}^{\gamma F-\gamma \theta_{0} \log K} \geq v\right] \leq C \exp \left\{-\left(2 \theta+\theta^{2} / 2\right) \log K\right\}=C \exp \left\{-\frac{2 \log v}{\gamma}-\frac{(\log v)^{2}}{2 \gamma^{2} \log K}\right\}=C v^{-2 / \gamma} \mathrm{e}^{-\frac{(\log v)^{2}}{2 \gamma^{2} \log K}},
$$

which is (2.9).

Finally, we will need a bound on the smoothness of the coarse field.

Lemma 2.9. There is a constant $C<\infty$ so that if $\mathbb{B} \subset \mathbb{B}^{*} \subset \mathbb{A}$ are boxes with aspect ratios between $1 / 3$ and 3 , then for all $\theta>0$ we have

$$
\mathbf{P}\left[\max _{x, y \in \mathbb{B}^{\circ}}\left|h_{\mathbb{A}: \mathbb{B}^{*}}(x)-h_{\mathbb{A}^{*}: \mathbb{B}^{*}}(y)\right| \geq \theta\right] \leq C \mathrm{e}^{-\theta^{2} / C} .
$$

Proof. By the Cauchy-Schwarz inequality and Lemma 2.6, we have

$$
\operatorname{Var}\left(\left(h_{\mathbb{A}: \mathbb{B}^{*}}(x)-h_{\mathbb{A}: \mathbb{B}^{*}}(y)\right)-\left(h_{\mathbb{A}: \mathbb{B}^{*}}\left(x^{\prime}\right)-h_{\mathbb{A}: \mathbb{B}^{*}}\left(y^{\prime}\right)\right)\right) \leq C \frac{|x-y|+\left|x^{\prime}-y^{\prime}\right|}{\operatorname{diam}_{\mathbb{E}} \mathbb{B}}
$$

for all $x, x^{\prime}, y, y^{\prime} \in \mathbb{B}^{\circ}$. The result follows from this, Lemma 2.6, Fernique's inequality, and the Borell-TIS inequality, similarly to the proof of Proposition 2.7.

\subsection{Liouville quantum gravity}

In this section we briefly review the properties of the Liouville quantum gravity measure. We first define the circle average process of a Gaussian free field. If $h_{\mathbb{A}}$ is a Gaussian free field on a box $\mathbb{A}$, then, for $x \in \mathbb{A}$ so that $\operatorname{dist}_{\mathrm{E}}(x, \partial \mathbb{A})>\varepsilon$, we define $h_{\mathbb{A}}^{\varepsilon}(x)$ to be the integral of $h_{\mathbb{A}}$ against the uniform measure on $\partial B(x, \varepsilon)$. The Liouville quantum gravity at inverse temperature $\gamma$ is then supposed to be the limit as $\varepsilon \downarrow 0$ of the random measure

$$
\mu_{h_{\AA}, \varepsilon}(\mathrm{d} x)=\varepsilon^{\gamma^{2} / 2} \mathrm{e}^{\gamma h_{\varepsilon}(x)} \mathrm{d} x
$$

Indeed, we have the following result of [17]:

Theorem 2.10. If $\gamma \in(0,2)$, then there is a random measure $\mu_{h_{\mathbb{A}}}$ such that, with probability 1 , we have

$$
\lim _{k \rightarrow \infty} \mu_{h_{\mathbb{A}}, 2^{-k}}=\mu_{h_{\mathbb{A}}}
$$

weakly.

The fact that the convergence in (2.11) is almost-sure will be important for us, because in our multiscale analysis we will often consider the Liouville quantum gravity on different boxes, with coupling induced by the coupling we have induced for the Gaussian free field. The almost-sure convergence means that this coupling induces a coupling on the corresponding LQG measures as well. 
Throughout the paper, we will treat $\gamma$ as a fixed constant in (0,2). To economize on indices, we will suppress $\gamma$ in the notation for LQG and subsequently defined objects. All constants throughout the paper will implicitly depend on $\gamma$. We will also fix throughout the paper a constant $\theta_{0}>2$ so that

$$
\eta:=\frac{2 \gamma \theta_{0}}{4+\gamma^{2}}<1
$$

$\eta$ will also be treated as a fixed constant throughout the paper. The reason for insisting that $\theta_{0}>2$ is to match with the condition in Corollary 2.8: we will treat $\theta_{0} \log K$ as the cutoff below which the "coarse field" at scale $K$ must be with high probability. The reason for insisting that $\eta<1$ is so that, according to Proposition 2.13 below, a sub-box of a box, once the maximum coarse field is considered, will "look like" a strictly smaller box than the larger box from the perspective of LQG measure.

We will also need the existence of positive and negative moments of the Liouville quantum gravity measure, which was proved in [21, 32]; see also [34, Theorems 2.11 and 2.12].

Proposition 2.11. There is a $v_{0}>1$ so that if $0 \leq v<v_{0}$, then for all domains $\mathbb{A} \subset \mathbf{R}^{2}$ we have

$$
\mathbf{E}\left[\mu_{h_{\mathbb{A}}}(\mathbb{A})^{v}\right]<\infty .
$$

Moreover, for any $0 \leq v<\infty$, we have for all domains $\mathbb{A}$ that

$$
\mathbf{E}\left[\mu_{h_{\mathbb{A}}}(\mathbb{A})^{-v}\right]<\infty .
$$

Proposition 2.12. Let $\mathbb{A}$ be a domain. It almost surely holds that for every $x \in \mathbb{A}$ and $r>0$ so that $B(x, r) \subset$ $\mathbb{A}$, we have $\mu_{h_{\mathbb{A}}}(B(x, r))>0$.

Proof. This follows from a simple union bound and (2.14).

If $\mu$ and $v$ are Radon measures on the same set $\mathbb{X}$, we say that $\mu \leq v$ if $v-\mu$ is also a measure (not just a signed measure). If $\mathbb{A} \subset \mathbb{B}$, then we have by (2.10) and the smoothness of $h_{\mathbb{B}: \mathbb{A}}$ that

$$
\left.\mu_{h_{\mathbb{B}}}\right|_{\mathbb{A}}=\exp \left\{\gamma h_{\mathbb{B}: \mathbb{A}}\right\} \mu_{h_{\mathbb{B}}}
$$

almost surely. (Here, $\left.\mu_{h_{\mathbb{B}}}\right|_{\mathbb{A}}$ denotes the measure $\mu_{h_{\mathbb{B}}}$ restricted to $\mathbb{A}$.) This implies that

$$
\left.\mu_{h_{\mathbb{B}}}\right|_{\mathbb{A}} \leq \exp \left\{\gamma \max _{x \in \mathbb{A}} h_{\mathbb{B}: \mathbb{A}}(x)\right\} \mu_{h_{\mathbb{B}}} .
$$

An important property of Liouville quantum gravity is the conformal covariance of the measure; see [5, Theorem 2.8]:

Proposition 2.13. Suppose that $\mathbb{V}$ and $\mathbb{V}^{\prime}$ are domains and $F: \mathbb{V} \rightarrow \mathbb{V}^{\prime}$ is a conformal homeomorphism. Then we have that

$$
\mu_{h_{\mathbb{V}}} \circ F^{-1}=\mathrm{e}^{\left(2+\gamma^{2} / 2\right) \log \left|\left(F^{-1}\right)^{\prime}\right|} \mu_{h_{\mathbb{V}} \circ F^{-1}} \stackrel{\operatorname{law}}{=} \mathrm{e}^{\left(2+\gamma^{2} / 2\right) \log \left|\left(F^{-1}\right)^{\prime}\right|} \mu_{h_{\mathbb{V}^{\prime}}} .
$$

\subsection{Metrics defined in terms of measures}

In this section we describe the process we use to construct a metric from a measure. The definitions and results in this section are purely deterministic. Of course, we plan to apply this construction to the case when the measure is a Liouville quantum gravity measure, which we will do in the next section, yielding the Liouville graph distance.

If $\mathbb{B}$ is a box, then define the space of paths $\mathcal{P}_{\mathbb{B}}$ to be the set of continuous images of $[0,1]$ in $\mathbb{B}$. If $x, y \in \mathbb{B}$, then define $\mathcal{P}_{\mathbb{B}}(x, y)=\left\{\pi \in \mathcal{P}_{\mathbb{B}} \mid x, y \in \pi\right\}$. Given a box $\mathbb{B}$ and a finite measure $\mu$ on $\mathbb{B}^{(R)}$, define

$$
\underline{\mathscr{B}}(\mu, \mathbb{B}, \delta)=\left\{\overline{B(x, r)} \mid r \in\left(0, \operatorname{diam}_{\mathrm{E}}(\mathbb{B})\right), x \in \mathbb{B}, \mu(B(x, r))<\delta\right\} .
$$

Here and throughout the paper, $B(x, r)$ denotes the open Euclidean ball with center $x$ and radius $r$. 
Definition 2.14. Let $\mathbb{B}$ be a domain, $\mu$ a measure on $\mathbb{B}, \delta>0$, and $R>0$. If $\mu$ is a measure on $\mathbb{B}^{(R)}$, then define the $\mu$-graph length of a path $\pi \in \mathcal{P}_{\mathbb{B}}$ as

$$
\underline{d}_{\mu, \mathbb{B}, \delta}(\pi)=\min \left\{n \in \mathbf{N}: \text { there are } B_{1}, \ldots, B_{n} \in \underline{\mathscr{B}}(\mu, \mathbb{B}, \delta) \text { such that } \pi \subset B_{1} \cup \cdots \cup B_{n}\right\} .
$$

We further define

$$
\underline{d}_{\mu, \mathbb{B}, \delta}(x, y)= \begin{cases}0, & x=y \\ \min _{\pi \in \mathcal{P}_{\mathbb{B}}(x, y)} \underline{d}_{\mu, \mathbb{B}, \delta}(\pi), & x \neq y .\end{cases}
$$

For the purposes of establishing measurability of the distances when the measure is random, we now show that the distance can be expressed as the minimum of countably many functions. This is a purely technical point.

Lemma 2.15. Let $\mathbb{B}$ be a domain, $\mu$ a measure on $\mathbb{B}$, and $\delta>0$. Define

$$
\stackrel{\mathscr{B}}{\mathbf{Q}}_{\mathbf{Q}}(\mu, \mathbb{B}, \delta)=\left\{\overline{B(x, r)} \mid r \in\left(0, \operatorname{diam}_{\mathrm{E}}(\mathbb{B})\right) \cap \mathbf{Q}, \mu(B(x, r))<\delta\right\} .
$$

Then we have that

$$
\underline{d}_{\mu, \mathbb{B}, \delta}(\pi)=\min \left\{n \in \mathbf{N}: \text { there are } B_{1}, \ldots, B_{n} \in \underline{\mathscr{B}}_{\mathbf{Q}}(\mu, \mathbb{B}, \delta) \text { such that } \pi \subset B_{1} \cup \cdots \cup B_{n}\right\} .
$$

Proof. This follows from the fact that if $B$ is a closed ball such that $\mu(B)<\delta$, then there is a ball $B^{\prime}$ containing $B$ so that $\mu\left(B^{\prime}\right)<\delta$ and $B^{\prime}$ has rational center and rational radius arbitrarily close to that of $B$.

It will be more convenient for us to work with a modified graph length, which has a somewhat better "continuity" property with respect to small perturbations of the field. (See equations (2.28) and (2.29) below.) We will see shortly (Proposition 2.25) that the modified graph length differs from the original one only by at most a factor of 2 . Define

$$
\mathscr{B}(\mathbb{B}, R)=\{\overline{B(x, r)} \mid r \in(0, R), x \in \mathbb{B}\} .
$$

Definition 2.16. For $\delta>0$, define

$$
\kappa_{\delta}(t)=\max \left\{1, \delta^{-1} t\right\} .
$$

If $\mathbb{B}$ is a domain and $\mu$ is a measure on $\mathbb{B}^{(R)}$, then define the $(\mu, \mathbb{B}, \delta, R)$-graph length of a path $\pi \in \mathcal{P}_{\mathbb{B}}$ as

$$
d_{\mu, \mathbb{B}, \delta, R}(\pi)=\inf \left\{\sum_{k=1}^{n} \kappa_{\delta}\left(\mu\left(B_{k}\right)\right): \text { there are } B_{1}, \ldots, B_{n} \in \mathscr{B}(\mathbb{B}, R) \text { such that } \pi \subset B_{1} \cup \cdots \cup B_{n}\right\} \text {. }
$$

We further define

$$
d_{\mu, \mathbb{B}, \delta, R}(x, y)= \begin{cases}0, & x=y \\ \min _{\pi \in \mathcal{P}_{\mathbb{B}}(x, y)} d_{\mu, \mathbb{B}, \delta, R}(\pi), & x \neq y .\end{cases}
$$

If $R>\operatorname{diam}_{\mathrm{E}}(\mathbb{B})$, then we define $d_{\mu, \mathbb{B}, \delta, R}=d_{\mu, \mathbb{B}, \delta, \operatorname{diam}_{\mathrm{E}}(\mathbb{B}) \text {, since any ball of radius greater than diam }}(\mathbb{B})$ can be replaced by one of radius less than $\operatorname{diam}_{E}(\mathbb{B})$ without changing the minimum.

Remark 2.17. The difference between the definitions of $d$ and $\underline{d}$ is that the definition of $d$ allows "too large" balls to be used, if one pays a "price." The price, however, is heuristically very steep, because one has to pay for the measure of the entire large ball, rather than just the smaller region around the path that would be required if one used smaller balls. Thus, we do not expect $d$ and $\underline{d}$ to differ by very much. The reason to use $d$ instead of $\underline{d}$ is that $d$ changes very little under small multiplicative perturbations of the measure (see (2.28) and (2.29) below), while $\underline{d}$ may change by up to a factor of 2 even if the measure is multiplied by $1+\varepsilon$ for $\varepsilon \ll 1$. 
Remark 2.18. The parameter $R$ of $d$ restricts the maximum Euclidean size of a ball that can be used to cover the path. If $\mathbb{B}$ and $R$ are of order 1 , and $\delta \ll 1$, then we would not expect $R$ to play any significant role, because balls of Euclidean radius of order 1 are extremely unlikely to have LQG mass $\delta$. However, it will often simplify our analysis to work with a metric in which we know that no balls of radius greater than $R$ are used-more importantly in order to use Proposition 2.29 below. We can then deal with the error incurred by this modification separately.

We prove a measurability lemma for $d$, analogous to Lemma 2.15 .

Lemma 2.19. Let $\mathbb{B}$ be a domain and $R>0$. Define

$$
\mathscr{B}_{\mathbf{Q}}(\mathbb{B}, R)=\left\{\overline{B(x, r)} \mid r \in(0, R) \cap \mathbf{Q}, x \in \mathbb{B} \cap \mathbf{Q}^{2}\right\} .
$$

Then we have that

$$
\underline{d}_{\mu, \mathbb{B}, \delta, R}(\pi)=\inf \left\{\sum_{k=1}^{n} \kappa_{\delta}\left(\mu\left(B_{k}\right)\right): \text { there are } B_{1}, \ldots, B_{n} \in \mathscr{B}(\mathbb{B}, R) \text { such that } \pi \subset B_{1} \cup \cdots \cup B_{n}\right\} .
$$

Proof. This follows from the fact that if $B$ is a closed ball, then for any $\varepsilon>0$ there is a ball $B^{\prime}$ containing $B$ so that $\mu\left(B^{\prime}\right)<\mu(B)+\varepsilon$ and $B^{\prime}$ has rational center and rational radius arbitrarily close to that of $B$.

\subsubsection{Notation for distances}

We will use a flexible notation for the geometrical notions of distance that we will use. For a box $\mathbb{B}$, we recall that $\mathrm{L}_{\mathbb{B}}, \mathrm{R}_{\mathbb{B}}, \mathrm{T}_{\mathbb{B}}$, and $\mathrm{B}_{\mathbb{B}}$ denote the left, right, top, and bottom edges of $\mathbb{B}$, respectively. Given a box $\mathbb{B}$, we will call the "easy direction" across $\mathbb{B}$ the direction between the longer sides of $\mathbb{B}$, and the "hard direction" across $\mathbb{B}$ the direction between the shorter sides of $\mathbb{B}$, as illustrated in Figure 1.1. Formally, let

$$
\left(\operatorname{easy}_{\mathbb{B}}, \operatorname{hard}_{\mathbb{B}}\right)= \begin{cases}\left(\left(\mathrm{L}_{\mathbb{B}}, \mathrm{R}_{\mathbb{B}}\right),\left(\mathrm{T}_{\mathbb{B}}, \mathrm{B}_{\mathbb{B}}\right)\right), & \operatorname{width}(\mathbb{B})<\operatorname{height}(\mathbb{B}) ; \\ \left(\left(\mathrm{T}_{\mathbb{B}}, \mathrm{B}_{\mathbb{B}}\right),\left(\mathrm{L}_{\mathbb{B}}, \mathrm{R}_{\mathbb{B}}\right)\right), & \operatorname{height}(\mathbb{B})<\operatorname{width}(\mathbb{B}) .\end{cases}
$$

In all of these notations, we will drop the subscript $\mathbb{B}$ when it is clear from context. We will often use this in notation like $d_{\mu, \mathbb{B}, \delta, R}(\mathrm{~L}, \mathrm{R})$, denoting the minimum $(\mu, \mathbb{B}, \delta, R)$-graph length of paths crossing from left to right in $\mathbb{B}$, or $d_{\mu, \mathbb{B}, \delta, R}$ (easy), denoting the minimum $(\mu, \mathbb{B}, \delta, \mathbb{R})$-graph length of an easy crossing of $\mathbb{B}$.

Also, define

$$
d_{\mu, \mathbb{B}, \delta, R}(\min ; a)=\min _{\substack{x, y \in \mathbb{B} \\|x-y| \geq a \operatorname{diam}_{\mathbb{B}} \mathbb{B}}} d_{\mu, \mathbb{B}, \delta, R}(x, y) .
$$

Suppose that $\mathbb{A}$ is the intersection of a rectangular annulus with a rectangle $\mathbb{R}$, both of whose side lengths are greater than the diameter of the annulus, so that either $\mathbb{A} \subset \mathbb{R}$ or $\mathbb{A} \cap \partial R$ has exactly two connected components. If $\mu$ is a measure on $\mathbb{A}^{(R)}$, define

$$
d_{\mu, \mathbb{A}, \delta, R}(\text { around })=\min _{\pi} d_{\mu, \mathbb{A}, \delta, R}(\pi),
$$

where $\pi$ ranges over all circuits around $\mathbb{A}$ if $\mathbb{A}$ is an annulus, or simply as

$$
d_{\mu, \mathbb{A}, \delta, R}(\text { around })=d_{\mu, \mathbb{A}, \delta, R}(X, Y),
$$

where $X$ and $Y$ are the two connected components of $\mathbb{A} \cap \partial \mathbb{R}$, if $\mathbb{A}$ is simply-connected. 


\subsubsection{Comparison inequalities}

The following comparison inequalities are immediate consequences of Definition 2.16:

1. If $\delta \leq \delta^{\prime}$, then

$$
d_{\mu, \mathbb{B}, \delta, R} \geq d_{\mu, \mathbb{B}, \delta^{\prime}, R}
$$

2. If $R^{\prime} \leq R$, then

$$
d_{\mu, \mathbb{B}, \delta, R} \leq d_{\mu, \mathbb{B}, \delta, R^{\prime}}
$$

3. For all $R>0$ we have

$$
d_{\mu, \mathbb{B}, \delta, R}=d_{\mu, \mathbb{B}, \delta, R \wedge \operatorname{diam}_{\mathrm{E}}(\mathbb{B})} .
$$

4. If $\left.\mu\right|_{\mathbb{B}(R)} \leq\left. v\right|_{\mathbb{B}(R)}$, then

$$
d_{\mu, \mathbb{B}, \delta, R} \leq d_{\nu, \mathbb{B}, \delta, R}
$$

5. If $\mathbb{B} \subset \mathbb{B}^{\prime}$, then

$$
d_{\mu, \mathbb{B}^{\prime}, \delta, R}(x, y) \leq d_{\mu, \mathbb{B}, \delta, R}(x, y)
$$

for all $x, y \in \mathbb{B}$.

6. For any $\alpha>0$, we have

$$
d_{\alpha \mu, \mathbb{B}, \delta, R}=d_{\mu, \mathbb{B}, \alpha^{-1} \delta, R} .
$$

If $\alpha \leq 1$, then

$$
d_{\mu, \mathbb{B}, \alpha \delta, R}(x, y) \leq \alpha^{-1} d_{\mu, \mathbb{B}, \delta, R}(x, y) .
$$

If $\alpha \geq 1$, then

$$
d_{\alpha \mu, \mathbb{B}, \delta, R} \leq \alpha d_{\mu, \mathbb{B}, \delta, R}(x, y) .
$$

7. The triangle inequality holds: if $\pi=\pi_{1} \cup \pi_{2}$, then

$$
d_{\mu, \mathbb{B}, \delta, R}(\pi) \leq d_{\mu, \mathbb{B}, \delta, R}\left(\pi_{1}\right)+d_{\mu, \mathbb{B}, \delta, R}\left(\pi_{2}\right) .
$$

Remark 2.20. All of the above properties with the exception of (2.28) and (2.29), as well as those involving $R$, also apply for $\underline{d}$. Note that $\underline{d}$ could have been defined using the $R$ parameter in the same way as $d$, and then (2.23) and (2.24) would hold for $\underline{d}$ as well, but we will not need this in the paper.

We will need a bound in the opposite direction for (2.23): a bound which controls how much the Liouville graph distance can increase when we decrease $R$, requiring the use of smaller balls. We will prove this as Lemma 2.23. This first requires the following lemma and definition.

Lemma 2.21. Suppose that $a_{1}+\cdots+a_{n} \leq b$. Then we have (recalling that $\kappa_{\delta}$ was defined in (2.18)) that

$$
\sum_{k=1}^{n} \kappa_{\delta}\left(a_{k}\right) \leq \kappa_{\delta}(b)+n .
$$

Proof. We have that $\delta^{-1} t \leq \kappa_{\delta}(t) \leq \delta^{-1} t+1$ for all $t$, so

$$
\sum_{k=1}^{n} \kappa_{\delta}\left(a_{k}\right) \leq \sum_{k=1}^{n}\left(\delta^{-1} a_{k}+1\right) \leq \delta^{-1} b+n \leq \kappa_{\delta}(b)+n .
$$


Definition 2.22. Suppose that $\mathbb{B}$ is a domain, $\mu$ is a measure on $\mathbb{B}$, and $\delta, R>0$. Let $x, y \in \mathbb{B}$ and suppose that $\pi$ is a path in $\mathbb{B}$ connecting $x$ and $y$. We will say that $\pi$ is a $(\mu, \mathbb{B}, \delta, R)$-geodesic if $\pi$ consists of a sequence of straight line segments between successive points $x, z_{1}, \ldots, z_{N}, y \in \mathbb{B}$ and there is a sequence of radii $r_{1}, \ldots, r_{N} \in(0, R)$ so that $\sum_{j=1}^{N} \mu\left(\overline{B\left(z_{i}, r_{i}\right)}\right)=d_{\mu, \mathbb{B}, \delta, R}(x, y)$ and $\pi \subset \bigcup_{j=1}^{N} \overline{B\left(z_{j}, r_{j}\right)}$. We will call $z_{1}, \ldots, z_{N}$ the corresponding geodesic points, $r_{1}, \ldots, r_{N}$ the corresponding geodesic radii, and $\overline{B\left(z_{1}, r_{1}\right)}, \ldots, \overline{B\left(z_{N}, r_{N}\right)}$ the corresponding geodesic balls.

Lemma 2.23. There is a constant $C$ so that if $B$ is a domain, $\mu$ is a measure on $\mathbb{B}, \delta>0$, and $0<R^{\prime} \leq R$, then

$$
d_{\mu, \mathbb{B}, \delta, R^{\prime}}(x, y) \leq d_{\mu, \mathbb{B}, \delta, R}(x, y)+C \frac{R \operatorname{Leb}\left(\mathbb{B}^{(R)}\right)}{\left(R^{\prime}\right)^{3}},
$$

where Leb denotes the Lebesgue measure, and also

$$
d_{\mu, \mathbb{B}, \delta, R^{\prime}}(x, y) \leq\left(1+C R / R^{\prime}\right) d_{\mu, \mathbb{B}, \delta, R}(x, y) .
$$

Proof. Let $x, y \in \mathbb{B}$. Let $B_{1}, \ldots, B_{N}$ be a set of geodesic balls for a geodesic between $x$ and $y$. We note that for any $1 \leq i<j<k \leq N$, we have that $B_{i} \cap B_{j} \cap B_{k}=\emptyset$; if not, then one of the balls could be eliminated to get a shorter distance. Therefore, if $S=\left\{i \in\{1, \ldots, N\} \mid \operatorname{diam}_{\mathrm{E}}\left(B_{i}\right) \geq R^{\prime}\right\}$, then we have that

$$
\frac{1}{2}|S|\left(R^{\prime}\right)^{2} \leq \sum_{i: \operatorname{diam}_{\mathrm{E}}\left(B_{i}\right) \geq R^{\prime}} \operatorname{Leb}\left(B_{i}\right) \leq 2 \operatorname{Leb}\left(\bigcup_{i: \operatorname{diam}_{\mathrm{E}}\left(B_{i}\right) \geq R^{\prime}} B_{i}\right) \leq 2 \operatorname{Leb}\left(\mathbb{B}^{(R)}\right),
$$

so

$$
|S| \leq 4 \pi \frac{\operatorname{Leb}\left(\mathbb{B}^{(R)}\right)}{\left(R^{\prime}\right)^{2}} .
$$

Now for each $i \in S, B_{i}$ can be replaced by a set of at most $2 \pi\left\lceil R / R^{\prime}\right\rceil$ subset balls of radius $R^{\prime}$, with centers in $\mathbb{B}$, so that a path from $x$ to $y$ in $\mathbb{B}$ is still covered, and the new balls in each older ball have total measure no larger than the measure of the original ball. This implies, by Lemma 2.21, that

$$
d_{\mu, \mathbb{B}, \delta, R^{\prime}}(x, y) \leq d_{\mu, \mathbb{B}, \delta, R}(x, y)+2 \pi\left\lceil R / R^{\prime}\right\rceil|S| \leq d_{\mu, \mathbb{B}, \delta, R}(x, y)+C \frac{R \operatorname{Leb}\left(\mathbb{B}^{(R)}\right)}{\left(R^{\prime}\right)^{3}}
$$

for some absolute constant $C$, which completes the proof of (2.31). On the other hand, (2.32) follows from (2.33) by the trivial bound $|S| \leq N$.

We will also want to relate our modified distances $d$ back to our original distances of interest $\underline{d}$. This turns out to be very simple, but we first require a definition.

Definition 2.24. By an admissible measure on a set $\mathbb{A}$ we will mean a nonatomic Radon measure $\mu$ such that for every $x \in \mathbb{A}$ and $r>0$ such that $B(x, r) \subset \mathbb{A}$, we have

$$
\mu(B(x, r))>0 .
$$

By Proposition 2.12, the Liouville quantum gravity measures are admissible almost surely. Note also that if $\mu$ is an admissible measure then $\alpha \mu$ is also an admissible measure for any $\alpha \in(0, \infty)$. Now we can state the relationship between $d$ and $\underline{d}$.

Proposition 2.25. If $\mu$ is an admissible measure on $\mathbb{B}$ and $\delta, R>0$, then

$$
d_{\mu, \mathbb{B}, \delta, R}(x, y) \leq \underline{d}_{\mu, \mathbb{B}, \delta, R}(x, y) \leq 2 d_{\mu, \mathbb{B}, \delta, R}(x, y) .
$$




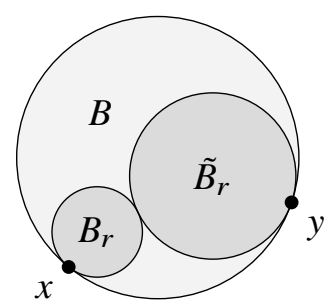

Figure 2.1: Construction in the proof of Lemma 2.26.

The first inequality is trivial by (2.18). The second follows from the following lemma.

Lemma 2.26. Suppose that $\mu$ is an admissible measure on $\mathbb{B}, \alpha \in(0,1), B$ is a closed ball so that $\mu(B)=\delta$, and $x, y \in \partial B$. Then there are closed balls $B^{\prime}, B^{\prime \prime} \subset B$ so that $\mu\left(B_{1}\right)<\alpha \delta, \mu\left(B_{2}\right)<(1-\alpha) \delta, B^{\prime} \cup B^{\prime \prime}$ is path-connected, and $x, y \in B_{1} \cup B_{2}$.

Proof. For $r \in[0,1]$, let $B_{r}$ be the closed ball of radius $r$ which is internally tangent to $B$ at $x$, so $B_{0}=\{x\}$ and $B_{1}=B$. Let $\tilde{B}_{r}$ be the closed ball which is internally tangent to $B$ at $y$ and externally tangent to $B_{r}$. (See Figure 2.1.) Let $f(r)=\mu\left(B_{r}\right)$ and let $\tilde{f}(r)=\mu\left(\tilde{B}_{r}\right)$. We note that $f$ is increasing and $\tilde{f}$ is decreasing. Since $\mu$ is nonatomic, we have that

$$
f(r)+\tilde{f}(r) \leq \mu(B)=\delta .
$$

We claim that $f$ is right-continuous. Indeed, if $r_{n} \downarrow r$, then we have

$$
\lim _{n \rightarrow \infty} f\left(r_{n}\right)=\mu\left(\bigcap_{n=1}^{\infty} B_{r_{n}}\right)=\mu\left(B_{r}\right) .
$$

Similarly, $\tilde{f}$ is left-continuous, because if $r_{n} \uparrow r$, then we have

$$
\lim _{n \rightarrow \infty} \tilde{f}\left(r_{n}\right)=\mu\left(\bigcap_{n=1}^{\infty} \tilde{B}_{r_{n}}\right)=\mu\left(\tilde{B}_{r}\right) .
$$

In particular, this implies that $f$ and $\tilde{f}$ are both upper-semicontinuous. Let

$$
r_{*}=\sup \{r \in[0,1] \mid f(r)<\alpha \delta\} .
$$

Because $f$ is upper-semicontinuous, we have that

$$
f\left(r_{*}\right) \geq \alpha \delta,
$$

so

$$
\tilde{f}\left(r_{*}\right) \leq(1-\alpha) \delta .
$$

Now if $\tilde{f}\left(r_{*}\right)=(1-\alpha) \delta$, then (2.36) implies that $f\left(r_{*}\right)=\alpha \delta$, so $\mu\left(B \backslash\left(B^{\prime} \cup B^{\prime \prime}\right)\right)=0$, contradicting the assumption that $\mu$ is admissible. Therefore, (2.37) implies that we must in fact have $\tilde{f}\left(r_{*}\right)<(1-\alpha) \delta$. By the left-continuity of $\tilde{f}$, there is an $r^{\prime}<r_{*}$ so that $\tilde{f}\left(r^{\prime}\right)<(1-\alpha) \delta$ as well, so since $r^{\prime}<r_{*}$, we must have that $f\left(r^{\prime}\right)<\alpha \delta$ by (2.35) and the fact that $f$ is increasing. Thus we can take $B^{\prime}=B_{r^{\prime}}$ and $B^{\prime \prime}=\tilde{B}_{r^{\prime}}$ to complete the proof.

Admissibility of a measure also implies that the crossing distance of a box can be made arbitrarily large by requiring the geodesic balls to have sufficiently small LQG measure. 
Lemma 2.27. If $\mu$ is an admissible measure on $\mathbb{B}$ and $R>0$, then

$$
\lim _{\delta \downarrow 0} d_{\mu, \mathbb{B}, \delta, R}(\mathrm{~L}, \mathrm{R})=\infty .
$$

Proof. For any $r>0$, there is a finite collection of balls $B_{1}, \ldots, B_{N}$ so that if $x \in \mathbb{B}$, then there is an $i \in$ $\{1, \ldots, N\}$ so that $B_{i} \subset B(x, r)$. Let $\delta_{0}=\min _{1 \leq i \leq N} \mu\left(B_{i}\right)$, which is positive by (2.34). Then if $\delta<\delta_{0}$, any ball of $\mu$-measure at most $\delta$ must have radius at most $r$, so

$$
d_{\mu, \mathbb{B}, \delta, R}(\mathrm{~L}, \mathrm{R}) \geq\left\lfloor r^{-1} \operatorname{dist}_{\mathrm{E}}\left(\mathrm{L}_{\mathbb{B}}, \mathrm{R}_{\mathbb{B}}\right)\right\rfloor,
$$

which implies the desired limit.

The last two propositions of this section will be used in lower bounds on the crossing distances. The first (Proposition 2.28) implies, informally, that if the geodesic between two points crosses a smaller box, then the two points must be at least as far apart as the crossing distance of the smaller box. The second (Proposition 2.29) extends this to the situation where a geodesic between two points crosses many smaller boxes, and says that the distance between the two points must be at least the sum of the crossing distances of the boxes. The caveat, however, is that the smaller boxes must be sufficiently separated so that a single geodesic ball cannot cover several of the smaller boxes. This is the primary importance of the parameter $R$ - it is used to prevent the use of geodesic balls with large Euclidean diameter that would cover many smaller boxes.

Proposition 2.28. Suppose that $\mathbb{C} \subset \mathbb{R}$ are boxes and $\mu$ is a measure on $\mathbb{R}^{(R)}$. Let $\pi$ be a $(\mu, \mathbb{R}, \delta, R)$-geodesic between two points in $\mathbb{R}$. Suppose that $w, z \in \mathbb{C} \cap \pi$, that $w$ appears before $z$ on the path $\pi$, and that the part of $\pi$ between $w$ and $z$ is completely contained in $\mathbb{C}$. Then

$$
d_{\mu, \mathbb{C}, \delta, R}(w, z)=d_{\mu, \mathbb{R}, \delta, R}(w, z) .
$$

Proof. By (2.26), it is sufficient to prove that

$$
d_{\mu, \mathbb{C}, \delta, R}(w, z) \leq d_{\mu, \mathbb{R}, \delta, R}(w, z) .
$$

Let $y_{1}, \ldots, y_{d}$ and $r_{1}, \ldots, r_{d}$ be the geodesic centers and radii for $\pi$, respectively. Let $y_{j}, \ldots, y_{k}$ be the minimal substring of the $y_{i}$ s so that the segment of $\pi$ between $w$ and $z$ is contained in $\overline{B\left(y_{j}, r_{j}\right)} \cup \cdots \cup \overline{B\left(y_{k}, r_{k}\right)}$. Then $d_{\mu, \mathbb{R}, \delta, R}(w, z)=\sum_{i=j}^{k} \kappa_{\delta}\left(\mu\left(\overline{B\left(y_{i}, r_{i}\right)}\right)\right)$. For each $j+1 \leq i \leq k-1$, we have $y_{i} \in \mathbb{C}$, so $\overline{B\left(y_{i}, r_{i}\right)} \in \mathscr{B}(\mathbb{C}, R)$. Let $u$ be the intersection of the segment between $y_{j}$ and $y_{j+1}$ with $\partial \mathbb{C}$. We can create a closed ball $B$ around $u$ that is contained in $\overline{B\left(y_{j}, r_{j}\right)}$ and internally tangent to $\overline{B\left(y_{j}, r_{j}\right)}$ at every element of $\pi \cap \mathbb{C} \cap \partial B\left(y_{j}, r_{j}\right)$ (which is a singleton unless $\left.y_{j} \in \partial \mathbb{C}\right)$. Then, we have $B \in \mathscr{B}(\mathbb{C}, R), \mu(B) \leq \mu\left(\overline{B\left(y_{j}, r_{j}\right)}\right)$, and $\pi \cap \overline{B\left(y_{j}, r_{j}\right)} \cap \mathbb{C} \subset B$. We can perform a similar construction around $u^{\prime}$, the intersection of the segment between $y_{k-1}$ and $y_{k}$ with $\partial \mathbb{C}$, to get a closed ball $B^{\prime} \in \mathscr{B}(\mathbb{C}, R)$ so that $\pi \cap \overline{B\left(y_{k}, r_{K}\right)} \cap \mathbb{C} \subset B^{\prime}$. (See Figure 2.2.) This completes the proof, since a path from $u$ to $u^{\prime}$ is contained in $B \cup \overline{B\left(y_{j+1}, r_{j+1}\right)} \cup \cdots \cup \overline{B\left(y_{k-1}, r_{k-1}\right)} \cup B^{\prime}$. (If $j=k$, then $B^{\prime}$ is not necessary and the construction still works.)

Proposition 2.29. Suppose that $\mathbb{C}_{1}, \ldots, \mathbb{C}_{J} \subset \mathbb{R}$ are boxes,

$$
R<\min _{1 \leq i, j \leq J} \operatorname{dist}_{\mathrm{E}}\left(\mathbb{C}_{i}, \mathbb{C}_{j}\right)
$$

and $\mu$ is a measure on $\mathbb{R}^{(R)}$. Let $x, y \in \mathbb{R}$ and let $\pi$ be $a(\mu, \mathbb{R}, \delta, R)$-geodesic between $x$ and $y$. Suppose that, for each $1 \leq i \leq j$, we have $w_{i}, z_{i} \in \mathbb{C}_{i} \cap \pi$, with $w_{i}$ appearing before $z_{i}$ on the path $\pi$, and the part of $\pi$ between $w_{i}$ and $z_{j}$ being completely contained in $\mathbb{C}_{i}$. Then

$$
d_{\mu, \mathbb{R}, \delta, R}(x, y) \geq \sum_{i=1}^{J} d_{\mu, \mathbb{R}, \delta, R}\left(w_{i}, z_{i}\right) .
$$




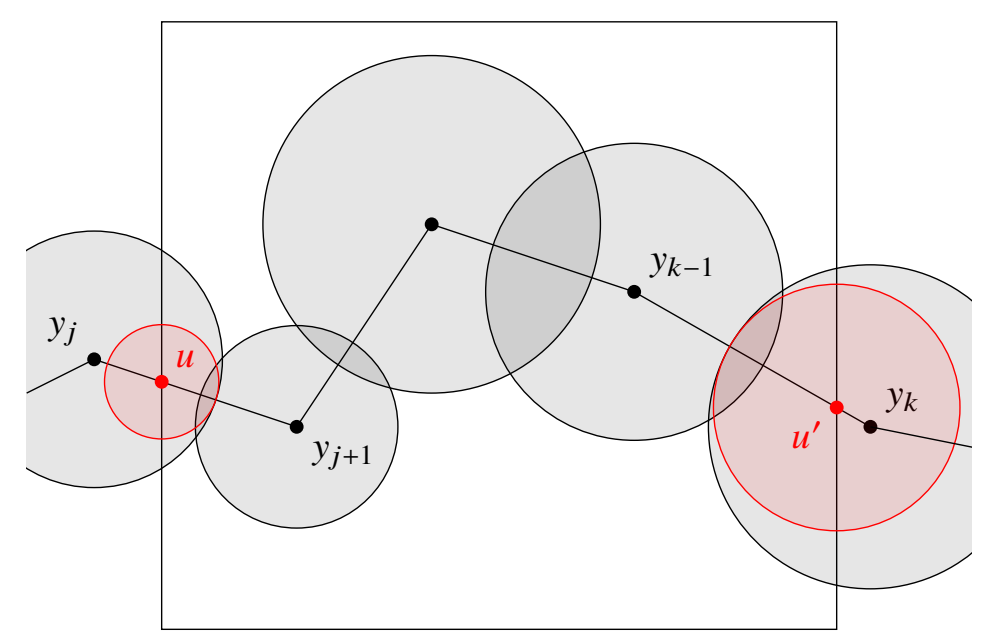

Figure 2.2: Construction in the proof of Proposition 2.28.

Proof. This follows from the proof of Proposition 2.28 when we note that the condition (2.39) implies that a single ball cannot intersect two distinct $\mathbb{C}_{i} \mathrm{~S}$.

\subsection{Liouville graph distance}

We now discuss the graph metric with respect to the Liouville quantum gravity measure: the Liouville graph distance.

Definition 2.30. If $\mathbb{A} \subset \mathbb{B}$ are boxes and $\delta>0, \gamma \in(0,2)$, and $R \leq \operatorname{dist}_{\mathrm{E}}(\mathbb{A}, \partial \mathbb{B})$, then we use the notation

$$
d_{\mathbb{A}, \mathbb{B}, \delta, R}=d_{\mu_{h, \gamma}, \mathbb{B}, \delta, R}
$$

To avoid a proliferation of subscripts, we will often abbreviate the notation for distances. If $\mathbb{A}$ is unspecified, we will mean that $\mathbb{A}=\mathbb{B}^{*}$. If $R$ is unspecified then we will mean that $R=\operatorname{diam}_{\mathbb{E}}(\mathbb{B}$ ) (which is a natural choice in light of (2.24)), and if in addition to $R$, the parameter $\delta$ is also unspecified, then we will mean that $\delta=1$. Thus we have

$$
\begin{aligned}
d_{\mathbb{A}, \mathbb{B}, \delta} & =d_{\mathbb{A}, \mathbb{B}, \delta, \operatorname{diam}_{\mathrm{E}}(\mathbb{B})}, \\
d_{\mathbb{B}, \delta} & =d_{\mathbb{B}}, \mathbb{B}, \delta, \operatorname{diam}_{\mathbb{E}}(\mathbb{B}), \\
d_{\mathbb{A}, \mathbb{B}} & =d_{\mathbb{A}, \mathbb{B}, 1, \operatorname{diam}_{\mathbb{E}}(\mathbb{B})}, \\
d_{\mathbb{B}} & =d_{\mathbb{B}}, \mathbb{B}, 1, \operatorname{diam}_{\mathbb{E}}(\mathbb{B}) .
\end{aligned}
$$

(Of course syntactically the second and third lines are ambiguous, but it will always be clear, from the font if nothing else, which variables represent boxes and which represent numbers, so there is no risk of confusion.)

Definition 2.31. We define $\Theta_{\mathbb{A}, \mathbb{B}, \delta, R}^{\mathrm{LR}}(p), \Theta_{\mathbb{A}, \mathbb{B}, \delta, R}^{\text {easy }}(p)$, and $\Theta_{\mathbb{A}, \mathbb{B}, \delta, R}^{\text {hard }}(p)$ to be the $p$ th quantiles of the random variables $d_{\mathbb{A}, \mathbb{B}, \delta, R}(\mathrm{~L}, \mathrm{R}), d_{\mathbb{A}, \mathbb{B}, \delta, R}\left(\right.$ easy), and $d_{\mathbb{A}, \mathbb{B}, \delta, R}($ hard$)$, respectively. We will abbreviate these notations in the same way as the $d$ notations.

We now establish several properties of the Liouville graph distance.

Proposition 2.32. If $\mathbb{B}^{(R)} \subset \mathbb{A}$ and $\delta, R>0$, then the processes

$$
\left\{\underline{d}_{\mathbb{A}, \mathbb{B}, \delta, R}(x, y)\right\}_{x, y \in \mathbb{B}}
$$


and

$$
\left\{d_{\mathbb{A}, \mathbb{B}, \delta, R}(x, y)\right\}_{x, y \in \mathbb{B}}
$$

are measurable with respect to the field $h_{\mathbb{A}}$.

Proof. This follows from Lemma 2.15 and Lemma 2.19, since both distances can be written as a minimum of countably many functions.

Proposition 2.33. If $\mathbb{A}_{1} \cap \mathbb{A}_{2}=\emptyset, R_{1}, R_{2}>0, \mathbb{B}_{i}^{\left(R_{i}\right)} \subset \mathbb{A}_{i}$, and $\delta_{i}>0$ for $i=1,2$, then $d_{\mathbb{A}_{1}, \mathbb{B}_{1}, \delta_{1}, R_{1}}$ and $d_{\mathbb{A}_{2}, \mathbb{B}_{2}, \delta_{2}, R_{2}}$ are independent.

Proof. This follows from the independence of the Gaussian free field on disjoint boxes (Proposition 2.2).

\subsubsection{Covariance properties of the Liouville graph distance}

The conformal covariance of the LQG measure heuristically implies conformal covariance of the Liouville graph distance. However, because the definition of Liouville graph distance specifies Euclidean balls, which are not in general taken by conformal maps to Euclidean balls, this is not exactly true. The next two propositions observe that the Liouville graph distance is covariant under Euclidean similarity transformations, which do of course takes Euclidean balls to Euclidean balls. The RSW result presented in Section 3 will rely on an approximate more general conformal covariance of the Liouville graph distance, which will be proved in that section.

Our first result is that the Liouville graph distance is invariant under Euclidean isometries.

Proposition 2.34. If $\mathbb{B} \subset \mathbb{A}, \delta, R>0$, and $f$ is a translation or rotation map, then

$$
\left\{d_{\mathbb{A}, \mathbb{B}, \delta, R}(x, y)\right\}_{x, y \in \mathbb{B}} \stackrel{\text { law }}{=}\left\{d_{f(\mathbb{A}), f(\mathbb{B}), \delta, R}(f(x), f(y))\right\}_{x, y \in \mathbb{B}} .
$$

Proof. This follows from the conformal covariance (Proposition 2.13) and the fact that translations and rotations take Euclidean balls to Euclidean balls.

Now we show how the Liouville graph distance transforms under scalings.

Proposition 2.35. If $\mathbb{B} \subset \mathbb{A}$ and $\delta, R>0$, then for any $\alpha>0$, we have

$$
\left\{d_{\alpha \mathbb{A}, \alpha \mathbb{B}, \alpha^{\gamma^{2} / 2+2} \delta, \alpha R}(\alpha x, \alpha y)\right\}_{x, y \in \mathbb{B}} \stackrel{\text { law }}{=}\left\{d_{\mathbb{A}, \mathbb{B}, \delta, R}(x, y)\right\}_{x, y \in \mathbb{B}} .
$$

Proof. Let $F: \mathbb{A} \rightarrow \alpha \mathbb{A}$ be given by scaling by $\alpha$. Then $F$ is a conformal map, so we have that

$$
\mu_{\alpha \mathbb{A}} \stackrel{\text { law }}{=} \alpha^{2+\gamma^{2} / 2}\left(\mu_{\mathbb{A}} \circ F^{-1}\right)
$$

by the conformal covariance (Proposition 2.13). Then we have, recalling (2.17), that

$$
\begin{aligned}
\mathscr{B}(\alpha \mathbb{B}, \alpha R) & =\{\overline{B(x, r)} \mid r \in(0, \alpha R), x \in \alpha \mathbb{B}\}=\left\{\alpha \overline{B\left(\alpha^{-1} x, \alpha^{-1} r\right)} \mid r \in(0, \alpha R), x \in \alpha \mathbb{B}\right\} \\
& =\{\alpha \overline{B(x, r)} \mid r \in(0, R), x \in \mathbb{B}\}=\alpha \mathscr{B}(\mathbb{B}, R),
\end{aligned}
$$

and that

$$
\left\{\kappa_{\alpha^{2+\gamma^{2} / 2} \delta}(\mu(\alpha B))\right\}_{B \in \mathscr{B}(\mathbb{B}, R)}=\left\{\kappa_{\delta}\left(\alpha^{-2-\gamma^{2} / 2} \mu(\alpha B)\right)\right\}_{B \in \mathscr{B}(\mathbb{B}, R)} \stackrel{\text { law }}{=}\left\{\kappa_{\delta}(\mu(B))\right\}_{B \in \mathscr{B}(\mathbb{B}, R)},
$$

which means that

$$
\left\{d_{\alpha \mathbb{A}, \alpha \mathbb{B}, \alpha^{\gamma^{2} / 2+2} \delta, \alpha R}(\alpha x, \alpha y)\right\}_{x, y \in \mathbb{B}} \stackrel{\text { law }}{=}\left\{d_{\mathbb{A}, \mathbb{B}, \delta, R}(x, y)\right\}_{x, y \in \mathbb{B}} .
$$


We note that a simple consequence of Proposition 2.35 and (2.22) is the following monotonicity of the quantiles:

Proposition 2.36. If $\alpha \geq 1$, then for all boxes $\mathbb{B} \subset \mathbb{A}$, all $p \in(0,1)$, and all $R>0$ we have

$$
\Theta_{\alpha \mathbb{A}, \alpha \mathbb{B}, 1, \alpha R}^{\mathrm{LR}}(p)=\Theta_{\mathbb{A}, \mathbb{B}, \alpha^{-\gamma^{2} / 2-2}, R}^{\mathrm{LR}}(p) \geq \Theta_{\mathbb{A}, \mathbb{B}, R}^{\mathrm{LR}}(p) .
$$

Lemma 2.37. For any $\mathbb{B} \subset \mathbb{A}$, any $p \in(0,1)$, and any $R>0$, we have

$$
\lim _{\delta \rightarrow 0} \Theta_{\mathbb{A}, \mathbb{B}, \delta, R}^{\mathrm{LR}}(p)=\infty .
$$

Proof. This follows from Lemma 2.27 and the fact that the LQG measure is admissible almost surely.

We also record a simple but useful consequence of the general facts established in Subsection 2.5.

Proposition 2.38. Suppose that $\mathbb{B} \subset \mathbb{B}^{\circ} \subset \mathbb{A} \subset \mathbb{A}^{\prime}$. We have

$$
d_{\mathbb{A}, \mathbb{B}, \delta^{\prime}, R} \leq d_{\mathbb{A}^{\prime}, \mathbb{B}, \delta, R} \leq d_{\mathbb{A}, \mathbb{B}, \delta^{\prime \prime}, R},
$$

where

$$
\begin{aligned}
\delta^{\prime} & =\exp \left\{-\gamma \min _{z \in \mathbb{B}^{\circ}} h_{\mathbb{A}^{\prime}: \mathbb{A}}(z)\right\} \delta, \\
\delta^{\prime \prime} & =\exp \left\{-\gamma \max _{z \in \mathbb{B}^{\circ}} h_{\mathbb{A}^{\prime}: \mathbb{A}}(z)\right\} \delta .
\end{aligned}
$$

Moreover, we have

$$
d_{\mathbb{A}^{\prime}, \mathbb{B}, \delta, R} \leq \exp \left\{\gamma\left(\max _{z \in \mathbb{B}^{\circ}} h_{\mathbb{A}^{\prime}: \mathbb{A}}(z)\right)^{+}\right\} d_{\mathbb{A}, \mathbb{B}, \delta, R}
$$

Proof. The inequalities in (2.41) follow immediately from (2.16) (relating the measures by multiplicative factors), (2.25) (going from a relationship between the measures to a relationship between the distances) and (2.27) (relating a constant factor in the measure to a constant factor in $\delta$ ). Then (2.42) is implied by (2.28).

Remark 2.39. We have two ways of understanding the effect on the distance when the measure is multiplied by a constant ("coarse field"): equality (2.27) (generally applied through (2.41)) and inequality (2.28) (often applied through (2.42)). As pointed out in Remark 2.17, the estimate (2.28) (and thus also the estimate (2.42)) is poor unless $\alpha$ is very close to 1 . When we perform estimates that are saturated as $\gamma \uparrow 2$, we thus always need to use (2.27) rather than (2.28). On the other hand, the change in $\delta$ in (2.27) is less suitable if we want to compare distances for the same path with respect to a slightly changed measure-in such cases, (2.28) will play a crucial role.

\section{The RSW theory}

In this section we give the proof of an RSW result, relating easy crossings to hard crossings in our setting. This was previously done in the high-temperature regime by the present authors in [8], using a framework used to prove an RSW result for Voronoi percolation in [41]. In the present paper, we follow the much simpler RSW proof method which was developed in [15] for first-passage percolation on fields with an approximate conformal invariance property. In this section, we adapt that proof to our setting.

The proof of the RSW result comes essentially in two steps. The first shows that the Liouville graph distance has an approximate conformal invariance property. This is the main difference between the proof 
we give here and that of [15]. In [15], the authors showed that the field on which they consider first-passage percolation changes only by a limited amount when it is compared to an appropriately coupled field on another domain, pulled back by a conformal isomorphism. In our setting, our underlying field (the GFF) is exactly conformally invariant. However, our definition of graph distance is based on Euclidean balls, which are not preserved by conformal transformations. Therefore, to show that the Liouville graph distance is approximately conformally invariant, we need to show that the Euclidean balls, when deformed by the conformal maps that we consider, can be replaced by only a constant number of Euclidean balls which lie inside the deformed balls.

The second step of the argument is then very similar to that of [15]. We consider a tall-and-skinny and a short-and-fat rectangle, and find a conformal isomorphism between them using the Riemann mapping theorem. (Actually, we need to use ellipses so that conformal maps have better smoothness properties, but these can be related back to rectangles by a simple estimate.) If we could map the vertical sides of one rectangle to the vertical sides of the other, then it would be clear how to map a left-right crossing of one rectangle to a left-right crossing of another. This is of course impossible, but we can solve the problem by finding some small segment of the right-hand side of the long rectangle which has a geodesic coming to it with positive probability, and can be mapped inside the right-hand side of the short rectangle. We explain the details in Subsection 3.2.

\subsection{Approximate conformal invariance}

The key ingredient of approximate conformal invariance in our setting is the following.

Proposition 3.1. Suppose that $\mathbb{U}$ and $\mathbb{V}$ are bounded domains (open subsets of $\mathbf{R}^{2}$ ) so that $\overline{\mathbb{U}} \subset \mathbb{V}$. Suppose further that $\mathbb{V}^{\prime}$ is another domain and $F: \mathbb{U} \rightarrow \mathbb{V}^{\prime}$ is a conformal homeomorphism. Finally, suppose that $R, R^{\prime}>0$. Then there is an $N=N\left(\mathbb{U}, \mathbb{V}, \mathbb{V}^{\prime}, F, R, R^{\prime}\right) \in \mathbf{N}$, such that for any closed ball $B \subset \mathbb{U}$ of radius at most $R$, any two points $y_{1}, y_{2} \in \partial F(B)$, and either connected component $X$ of $\partial F(B) \backslash\left\{y_{1}, y_{2}\right\}$, we have a sequence of closed balls $B_{1}, \ldots, B_{N}$ so that $B_{1} \cup \cdots \cup B_{N}$ is connected, $y_{1}, y_{2} \in B_{1} \cup \cdots \cup B_{N}, B_{1} \cup \cdots \cup B_{N} \subset F(B)$, and

$$
\sup _{x \in B_{1} \cup \cdots \cup B_{N}} \operatorname{dist}_{\mathrm{E}}(x, X) \leq R^{\prime}
$$

Moreover, we can take

$$
N\left(\mathbb{U}, \mathbb{V}, \mathbb{V}^{\prime}, F, R, R^{\prime}\right)=N\left(\alpha \mathbb{U}, \alpha \mathbb{V}, \mathbb{V}^{\prime}, x \mapsto F(x / \alpha), \alpha R, R^{\prime}\right)=N\left(\mathbb{U}, \mathbb{V}, \alpha \mathbb{V}^{\prime}, x \mapsto \alpha F(x), R, \alpha R^{\prime}\right)
$$

for any $\alpha>0$.

The statement of Proposition 3.1 has an intuitive interpretation. It says that a given conformal homeomorphism $F$, on a compact set inside of its domain, cannot disturb the geometry of a ball $B$ so violently that an unbounded number of balls inside of $F(B)$ are required to connect two points on the boundary of $F(B)$. This is essentially true because the derivatives of the conformal homeomorphism and its inverse must be bounded on a compact set in the interior of the domain. Quantifying this intuition, however, requires a bit of work. We first prove the following lemma.

Lemma 3.2. Suppose that $\xi:[-1,1] \rightarrow \mathbf{R}^{2}$ is a smooth curve and $x \in \mathbf{R}^{2}$ is such that $x-\xi(0)$ is perpendicular to $\xi^{\prime}(0)$. If

$$
|x-\xi(0)|<\frac{\left|\xi^{\prime}(0)\right|^{2}}{2\left\|\xi^{\prime \prime}\right\|_{\infty}},
$$

then

$$
|x-\xi(s)| \geq|x-\xi(0)|
$$


for all s satisfying

$$
|s| \leq \frac{\left|\xi^{\prime}(0)\right|}{2\left\|\xi^{\prime \prime}\right\|_{\infty}} .
$$

Proof. Put $v=\left|\xi^{\prime}(0)\right|$ and $d=|x-\xi(0)|$. We may change coordinates to assume that $\xi(0)=0, \xi^{\prime}(0)=(v, 0)$, and $x=(0, d)$. Then we have that

$$
\begin{aligned}
|x-\xi(s)|^{2} & =\xi_{1}(s)^{2}+\left(d-\xi_{2}(s)\right)^{2}=\left(v s+\int_{0}^{s}(s-r) \xi_{1}^{\prime \prime}(r) \mathrm{d} r\right)^{2}+\left(d-\int_{0}^{s}(s-r) \xi_{2}^{\prime \prime}(r) \mathrm{d} r\right)^{2} \\
& \geq d^{2}+v^{2} s^{2}+2 \int_{0}^{s}(s-r)\left[v s \xi_{1}^{\prime \prime}(r)-d \xi_{2}^{\prime \prime}(r)\right] \mathrm{d} r \geq d^{2}+s^{2}\left(v^{2}-(v s+d) \sup _{|r| \leq s}\left|\xi^{\prime \prime}(r)\right|\right) .
\end{aligned}
$$

We note that

$$
(v s+d) \sup _{|r| \leq s}\left|\xi^{\prime \prime}(r)\right| \leq(v s+d)\left\|\xi^{\prime \prime}\right\|_{\infty}<v^{2},
$$

where the last inequality is by (3.3) and (3.4), so in fact we have $|x-\xi(s)|^{2}>|x-\xi(0)|^{2}$, as claimed.

Now we can prove Proposition 3.1.

Proof of Proposition 3.1. Let $x$ and $r$ be the center and radius of $B$, respectively, so $B=\overline{B(x, r)}$. Parameterize $\partial B$ by

$$
\xi(t)=x+r(\cos t, \sin t) .
$$

Then $F \circ \xi$ is a parameterization of $\partial F(B)$. We have, using the fact that $F$ is conformal, that

$$
\left|(F \circ \xi)^{\prime}(t)\right|=\left|F^{\prime}(\xi(t)) \cdot \xi^{\prime}(t)\right| \in\left[R\left\|\left(F^{\prime}\right)^{-1}\right\|_{L^{\infty}(\mathbb{U})}^{-1}, r\left\|F^{\prime}\right\|_{L^{\infty}(\mathbb{U})}\right]
$$

and

$$
\begin{aligned}
\left|(F \circ \xi)^{\prime \prime}(t)\right|=\left.\left|F^{\prime \prime}(\xi(t)) \cdot\right| \xi^{\prime}(t)\right|^{2}+F^{\prime}(\xi(t)) \cdot \xi^{\prime \prime}(t) \mid & \leq r^{2}\left\|F^{\prime \prime}\right\|_{L^{\infty}(\mathbb{U})}+r\left\|F^{\prime}\right\|_{L^{\infty}(\mathbb{U})} \\
& \leq r R\left\|F^{\prime \prime}\right\|_{L^{\infty}(\mathbb{U})}+r\left\|F^{\prime}\right\|_{L^{\infty}(\mathbb{U})} .
\end{aligned}
$$

Let

$$
t_{0}=\frac{\left\|\left(F^{\prime}\right)^{-1}\right\|_{L^{\infty}(\mathbb{U})}^{-1}}{R\left\|F^{\prime \prime}\right\|_{L^{\infty}(\mathbb{U})}+\left\|F^{\prime}\right\|_{L^{\infty}(\mathbb{U})}}, \quad \quad \ell_{0}=\frac{r\left\|\left(F^{\prime}\right)^{-1}\right\|_{L^{\infty}(\mathbb{U})}^{-2}}{R\left\|F^{\prime \prime}\right\|_{L^{\infty}(\mathbb{U})}+\left\|F^{\prime}\right\|_{L^{\infty}(\mathbb{U})}} .
$$

Define, for $a>0$ to be fixed later,

$$
\omega_{a}(t)=F(\xi(t))+a \frac{\mathcal{R}(F \circ \xi)^{\prime}(t)}{r\left\|F^{\prime}\right\|_{L^{\infty}(\mathbb{U})}},
$$

where $\mathcal{R}$ denotes counterclockwise rotation by $\pi / 2$. Let

$$
b(t)=\left|\omega_{a}(t)-F(\xi(t))\right|=\left|a \frac{\mathcal{R}(F \circ \xi)^{\prime}(t)}{r\left\|F^{\prime}\right\|_{L^{\infty}(\mathbb{U})}}\right|=a \frac{\left|(F \circ \xi)^{\prime}(t)\right|}{r\left\|F^{\prime}\right\|_{L^{\infty}(\mathbb{U})}} .
$$

Fix $t \in[0,2 \pi]$. By (3.5), we have that

$$
b(t) \leq a .
$$

Thus, by Lemma 3.2 (applied with $x=\omega_{a}(t), \xi(s)=F(\xi(s-t))$ ), (3.5), and (3.6), if $a \leq \ell_{0}$ and $s \in\left[t-t_{0}, t+t_{0}\right]$ then

$$
\left|F(\xi(s))-\omega_{a}(t)\right| \geq b(t)
$$


Moreover, we know that if $x \in \partial B \backslash \xi\left(\left[t-t_{0}, t+t_{0}\right]\right)$, then

$$
|F(x)-F(\xi(t))| \geq \operatorname{cr}\left\|\left(F^{\prime}\right)^{-1}\right\|_{L^{\infty}(\mathbb{U})}^{-1} t_{0}
$$

for some absolute constant $c$. Let $a=r a_{0}$, where

$$
a_{0}=\min \left\{r^{-1} \ell_{0}, \frac{1}{2} c\left\|\left(F^{\prime}\right)^{-1}\right\|_{L^{\infty}(\mathbb{U})}^{-1} t_{0}, \frac{R^{\prime}}{2 r}\right\} .
$$

Then we have, for $s \notin\left[t-t_{0}, t+t_{0}\right]+2 \pi \mathbf{Z}$,

$$
\left|F(\xi(s))-\omega_{a}(t)\right| \geq|F(\xi(s))-F(\xi(t))|-\left|F(\xi(t))-\omega_{a}(t)\right| \geq c r\left\|\left(F^{\prime}\right)^{-1}\right\|_{L^{\infty}(\mathbb{U})}^{-1} t_{0}-a \geq a \geq b(t),
$$

where the first inequality is the triangle inequality, the second is by (3.11) and (3.9), the third is by (3.12), and the fourth is by (3.9). Together, (3.10) and (3.13) mean that (3.10) hold for all $s, t$. This implies that

$$
\overline{B\left(\omega_{a}(s), b(s)\right)} \subseteq \overline{F(B)}
$$

for all $s$. Note that $a_{0}$ only depends on $\mathbb{U}, \mathbb{V}, F, R, R^{\prime}$ and not on $B$, and moreover is invariant under the scalings indicated in (3.2).

We can also apply the triangle inequality to (3.7) to obtain, for all $s, s^{\prime}$,

$$
\begin{aligned}
\left|\omega_{a}(s)-\omega_{a}\left(s^{\prime}\right)\right| & \leq\left|F(\xi(s))-F\left(\xi\left(s^{\prime}\right)\right)\right|+\frac{a}{r\left\|F^{\prime}\right\|_{L^{\infty}(\mathbb{U})}}\left|(F \circ \xi)^{\prime}(s)-(F \circ \xi)^{\prime}\left(s^{\prime}\right)\right| \\
& \leq r\left\|F^{\prime}\right\|_{L^{\infty}(\mathbb{U})}\left|s-s^{\prime}\right|+\frac{a_{0}}{\left\|F^{\prime}\right\|_{L^{\infty}(\mathbb{U})}}\left(r R\left\|F^{\prime \prime}\right\|_{L^{\infty}(\mathbb{U})}+r\left\|F^{\prime}\right\|_{L^{\infty}(\mathbb{U})}\right)\left|s-s^{\prime}\right| \\
& =r\left(\left\|F^{\prime}\right\|_{L^{\infty}(\mathbb{U})}+a_{0}\left(R\left\|F^{\prime \prime}\right\|_{L^{\infty}(\mathbb{U})}\left\|F^{\prime}\right\|_{L^{\infty}(\mathbb{U})}^{-1}+1\right)\right)\left|s-s^{\prime}\right|,
\end{aligned}
$$

where in the second inequality we used (3.5) and (3.6) . On the other hand, we can compute from (3.8) and (3.5) that

$$
b(t)=a \frac{\left|(F \circ \xi)^{\prime}(t)\right|}{r\left\|F^{\prime}\right\|_{L^{\infty}(\mathbb{U})}} \geq \frac{a_{0} r}{\left\|\left(F^{\prime}\right)^{-1}\right\|_{L^{\infty}(\mathbb{U})}\left\|F^{\prime}\right\|_{L^{\infty}(\mathbb{U})}} .
$$

Therefore, if we define

$$
\iota=\frac{2 a_{0}\left(\left\|\left(F^{\prime}\right)^{-1}\right\|_{L^{\infty}(\mathbb{U})}\left\|F^{\prime}\right\|_{L^{\infty}(\mathbb{U})}\right)^{-1}}{\left\|F^{\prime}\right\|_{L^{\infty}(\mathbb{U})}+a_{0}\left(R\left\|F^{\prime \prime}\right\|_{L^{\infty}(\mathbb{U})}\left\|F^{\prime}\right\|_{L^{\infty}(\mathbb{U})}^{-1}+1\right)},
$$

then whenever $\left|s-s^{\prime}\right| \leq \iota$, we have

$$
\left|\omega_{a}(s)-\omega_{a}\left(s^{\prime}\right)\right| \leq b(s)+b\left(s^{\prime}\right)
$$

by (3.15) and (3.16). In particular, this means that

$$
\overline{B\left(\omega_{a}(s), b(s)\right)} \cap \overline{B\left(\omega_{a}\left(s^{\prime}\right), b\left(s^{\prime}\right)\right)} \neq \emptyset .
$$

Note that, like $a_{0}$, the constant $\iota$ depends only on $\mathbb{U}, \mathbb{V}, F, R, R^{\prime}$ and not on $B$, and is invariant under the scalings indicated in (3.2). Now if $s_{0}, s_{1} \in \mathbf{R}$ with $s_{1} \in\left(s_{0}, s_{0}+2 \pi\right)$, then we can choose a sequence of points

$$
s_{0}=t_{0}<t_{1}<t_{2}<\cdots<t_{N}=s_{1}
$$

so that $t_{i}-t_{i-1}<\iota$ for each $i$, and $N \leq 2 \pi / \iota$, which is invariant under the scalings indicated in (3.2) because $\iota$ is. Then we claim that

$$
\left\{\overline{B\left(\omega_{a}\left(t_{i}\right), b\left(t_{i}\right)\right)}: i \in\{0, \ldots, N\}\right\}
$$

is a set of balls as claimed in the lemma if $s_{1}$ and $s_{2}$ are chosen so that $\left\{y_{1}, y_{2}\right\}=\left\{\xi\left(s_{1}\right), \xi\left(s_{2}\right)\right\}$ and $X=$ $\xi\left(\left(s_{1}, s_{2}\right)\right)$. (See Figure 3.1.) The fact that the balls all lie inside $\overline{F(B)}$ is $(3.14)$, the fact that their union is connected is (3.17), and the fact that they have radius at most $R^{\prime}$, as well as (3.1), comes from (3.8) and (3.12). 


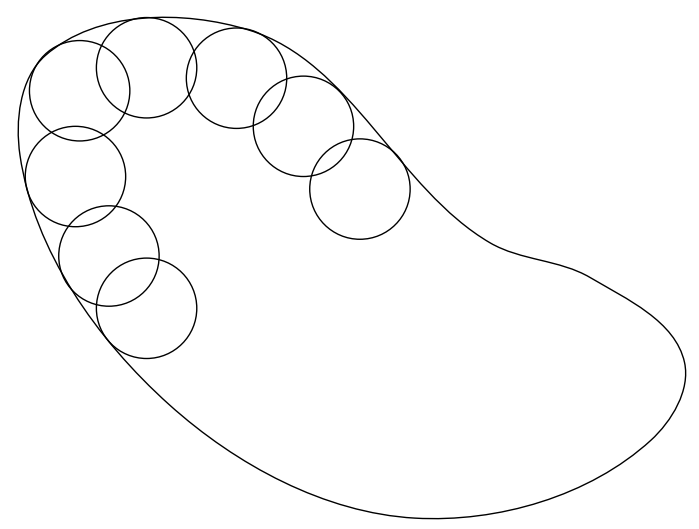

Figure 3.1: Connecting two points on the boundary of a deformed ball $F(B)$ with smaller Euclidean balls along the boundary. The analysis in the proof of Proposition 3.1 essentially amounts to ensuring that each smaller ball has sufficiently small radius of curvature to "locally" fit inside of $F(B)$, and sufficiently small diameter to "globally" fit inside of $F(B)$.

Corollary 3.3. Suppose that $\mathbb{U}$ and $\mathbb{V}$ are bounded open subsets of $\mathbf{R}^{2}$ so that $\overline{\mathbb{U}} \subset \mathbb{V}$. Suppose further that $\mathbb{V}^{\prime}$ is another domain and $F: \mathbb{V} \rightarrow \mathbb{V}^{\prime}$ is a conformal homeomorphism. Define

$$
\begin{aligned}
R & \in\left(0, \frac{1}{4} \operatorname{dist}_{\mathrm{E}}(\mathbb{U}, \partial \mathbb{V})\right), \\
R^{\prime} & \in\left(0, \frac{1}{4} \operatorname{dist}_{\mathrm{E}}\left(F(\mathbb{U}), \partial \mathbb{V}^{\prime}\right)\right)
\end{aligned}
$$

Suppose further that $\partial B(x, r) \cap \mathbb{U}$ is connected for every $x \in \mathbf{R}^{2}, r \in(0, R)$. Then, for all $x, y \in \mathbb{U}$, we have

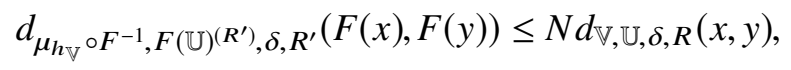

where

$$
N=N\left(\mathbb{U}, \mathbb{V}, \mathbb{V}^{\prime}, F, R, R^{\prime}\right)
$$

is as in the statement of Proposition 3.1.

Proof. Let $d=d_{\mathbb{V}, \mathbb{U}, \delta, R}(x, y)$. There is a path $\pi \in \mathcal{P}_{\mathbb{U}}(x, y)$ and a sequence of closed balls $B_{1}, \ldots, B_{n} \subset \mathbb{U}^{(2 R)}$ so that

$$
d=\sum_{j=1}^{n} \kappa_{\delta}\left(\mu_{h_{\mathbb{V}}}\left(B_{i}\right)\right)
$$

and $\pi \subset B_{1} \cup \cdots \cup B_{d}$. Now by Proposition 3.1, for each $1 \leq j \leq d$ there is a sequence of closed balls $B_{j ; 1}, \ldots, B_{j ; N} \subseteq F\left(B_{j}\right)$, where $N$ depends only on $\mathbb{U}, \mathbb{V}, \mathbb{V}^{\prime}, F, R, R^{\prime}$, so that if $P_{j}=B_{j ; 1} \cup \cdots \cup B_{j ; N}$ and $P=P_{1} \cup \cdots \cup P_{d}$, then $P$ is connected and $F(x), F(y) \in P$. Moreover, $B_{j ; 1}, \ldots, B_{j ; N}$ can be chosen to lie in $F(\mathbb{U})^{\left(R^{\prime}\right)}$ by (3.1) and the assumption that $\partial B_{j} \cap \mathbb{U}$ is connected. In particular, we have that

$$
\mu_{h_{\mathbb{V}}}\left(F^{-1}\left(B_{j ; i}\right)\right) \leq \mu_{h_{\mathbb{V}}}\left(B_{j}\right) \leq \delta .
$$

Therefore, by Lemma 2.21, we have that

$$
d_{\mu_{h_{\mathbb{V}}} \circ F^{-1}, F(\mathbb{U})^{\left(R^{\prime}\right), \delta, R}}(F(x), F(y)) \leq N d_{\mathbb{V}, \mathbb{U}, \delta, R}(x, y),
$$

as claimed. 


\subsection{The RSW result}

Now we can prove our RSW result. In this section, in order to access the conformal mappings that we will need to make the argument work, it will be more convenient to work with ellipses rather than rectangles. Define $\mathbb{E}\left(S_{1}, S_{2}\right)$ to be the filled, closed ellipse centered at 0 with horizontal axis of length $S_{1}$ and vertical axis of length $S_{2}$; that is,

$$
\mathbb{E}\left(S_{1}, S_{2}\right)=\left\{\left(x_{1}, x_{2}\right) \in \mathbf{R}^{2}:\left(2 x_{1} / S_{1}\right)^{2}+\left(2 x_{2} / S_{2}\right)^{2} \leq 1\right\} .
$$

Theorem 3.4. Let $\mathbb{R}_{1}=\mathbb{B}(1,2)$ and $\mathbb{R}_{2}=\mathbb{B}(2,1)$. There is a constant $c>0$ so that, for any $w>0$, we have

$$
\mathbf{P}\left(d_{\mathbb{R}_{2}, \delta}(\mathrm{L}, \mathrm{R}) \leq w\right) \geq c \mathbf{P}\left(d_{\mathbb{R}_{1}, \delta}(\mathrm{L}, \mathrm{R}) \leq c w\right) .
$$

Thus we have a constant $C_{\mathrm{RSW}}<\infty$ so that for any $p \in\left(0, C_{\mathrm{RSW}}\right)$,

$$
\Theta_{\mathbb{R}_{1}, \delta, R}^{\text {hard }}(p) \leq C_{\mathrm{RSW}} \Theta_{\mathbb{R}_{1}, \delta, R}^{\text {easy }}\left(C_{\mathrm{RSW}} p\right)
$$

Proof. Define

$$
q_{0}=\mathbf{P}\left[d_{\mathbb{R}_{1}, \delta}(\mathrm{L}, \mathrm{R}) \leq w\right] .
$$

Let $\mathbb{E}_{1}=\mathbb{E}(1,3)$ and $\mathbb{E}_{2}=\mathbb{E}(3,3 / 4)$. Let $X$ and $Y$ be the left and right, respectively, connected components of $\partial \mathbb{E}_{1} \cap \mathbb{R}_{1}$, and let $x_{0}$ and $x_{1}$ be the bottom and top, respectively, endpoints of $X$. By the Riemann mapping theorem and Schwarz reflection, for any $y \in Y$ there are open sets $\mathbb{F}_{1}, \mathbb{F}_{2}(y)$ and a conformal homeomorphism $F_{y}: \mathbb{F}_{1} \rightarrow \mathbb{F}_{2}(y)$ such that the following properties hold:

1. We have $\mathbb{E}_{1} \subset \mathbb{F}_{1} \subset \mathbb{R}_{1}^{*}, \mathbb{E}_{2} \subset \mathbb{E}_{2}(y) \subset \mathbb{R}_{2}^{*}$, and $F_{y}\left(\mathbb{E}_{1}\right)=\mathbb{E}_{2}$.

2. $F_{y}\left(x_{0}\right)$ and $F_{y}\left(x_{1}\right)$ are the upper- and lower-left, respectively, points of $\partial \mathbb{E}_{2} \cap \partial \mathbb{R}_{2}$.

3. $F_{y}(y)$ is the lower-right point of $\partial \mathbb{E}_{2} \cap \partial \mathbb{R}_{2}$.

4. We have that

$$
Q:=\sup _{y \in Y} \max \left\{\left\|F_{y}^{\prime}\right\|_{L^{\infty}\left(\mathbb{E}_{1}\right)},\left\|\left(F_{y}^{\prime}\right)^{-1}\right\|_{L^{\infty}\left(\mathbb{E}_{2}\right)}\right\}<\infty .
$$

(Here, $\left(F_{y}^{\prime}\right)^{-1}(x)=\left(F_{y}^{\prime}(x)\right)^{-1}$.)

This construction was used in the proof of [15, Theorem 3.1]. We note that condition 2 implies that $F_{y}(X)$ is the left connected component of $\overline{\mathbb{E}_{2} \backslash \mathbb{R}_{2}}$. See Figure 3.2 for a partial illustration of this setup.

Let $R_{1}=\frac{1}{4} \operatorname{dist}\left(\mathbb{E}_{1}, \partial \mathbb{F}_{1}\right)$. Now, on the event $E=\left\{\max _{z \in \mathbb{E}_{1}^{\left(R_{1}\right)}} h_{\mathbb{R}_{1}^{*}: \mathbb{F}_{1}}(z) \leq 0\right\}$ we have that

$$
d_{\mathbb{F}_{1}, \mathbb{E}_{1}, \delta, R_{1}}(X, Y) \leq d_{\mathbb{R}_{1}, \delta, R_{1}}(X, Y) \leq C d_{\mathbb{R}_{1}, \delta}(X, Y),
$$

where the second inequality is by (2.32). (Here we have folded the geometrical factor in (2.32) into the constant $C$.) Now we note that $d_{\mathbb{F}_{1}, \mathbb{E}_{1}, \delta, R_{1}}(X, Y)$ and $E$ are independent. Let $q_{*}=\mathbf{P}[E]$. We note that $q_{*}$ is strictly positive because $\mathbb{E}_{1}^{\left(R_{1}\right)}$ is separated from $\partial \mathbb{F}_{1}$ by a positive Euclidean distance and thus $\left.h_{\mathbb{R}_{1}^{*}: \mathbb{F}_{1}}\right|_{\mathbb{E}_{1}^{\left(R_{1}\right)}}$ is a uniformly smooth centered Gaussian process on a compact domain-thus there is a positive probability that it is nonpositive on $\mathbb{E}_{1}^{\left(R_{1}\right)}$. Then we have that

$$
\mathbf{P}\left(d_{\mathbb{F}_{1}, \mathbb{E}_{1}, \delta, R_{1}}(X, Y) \leq C w\right) \geq q_{*} q_{0} .
$$




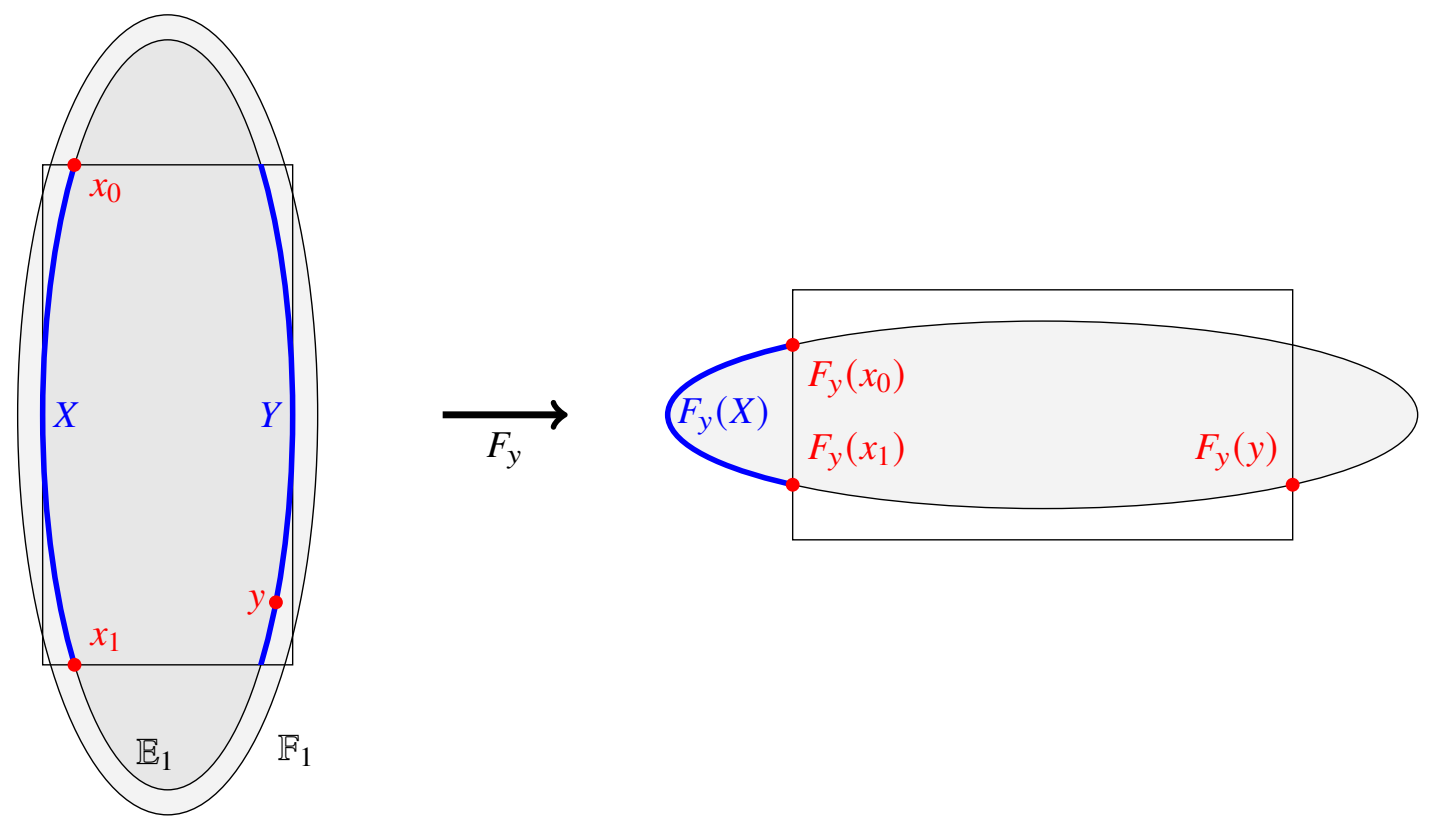

Figure 3.2: Illustration of the geometrical setup for the proof of Theorem 3.4.

Now write $Y$ as a union of disjoint curvilinear segments $Y_{1}, \ldots, Y_{M}$ so that $\operatorname{diam}_{\mathrm{E}}\left(Y_{k}\right) \leq(2 Q)^{-1}$, where $Q$ is as in (3.20) and $M$ is taken to satisfy $M \leq 3 Q$. By (3.21), we have that

$$
q_{*} q_{0} \leq \sum_{k=1}^{M} \mathbf{P}\left(d_{\mathbb{F}_{1}, \mathbb{E}_{1}, \delta, R_{1}}(X, Y) \leq C w\right),
$$

so there is some $1 \leq k \leq M$ so that

$$
\mathbf{P}\left(d_{\mathbb{F}_{1}, \mathbb{E}_{1}, \delta, R_{1}}\left(X, Y_{k}\right) \leq C w\right) \geq \frac{q_{*} q_{0}}{M} \geq \frac{q_{*} q_{0}}{3 Q} .
$$

Fix $y$ to be the bottom endpoint of $Y_{k}$. By (3.20), we observe that $F_{y}\left(Y_{k}\right)$ is contained in the right-hand connected component of $\mathbb{E}_{2} \backslash \mathbb{R}_{2}$. Let

$$
R_{2}=\min \left\{\frac{1}{4} \operatorname{dist}\left(\mathbb{E}_{2}, \partial \mathbb{F}_{2}(y)\right), \frac{1}{32}\right\}
$$

and define $\widetilde{\mathbb{E}}_{2}=\mathbb{E}_{2}^{\left(R_{2}\right)}$. By Corollary 3.3, we have an $N<\infty$ so that

$$
d_{\mu_{\mathbb{F}_{1}} \circ F_{y}^{-1}, \widetilde{\mathbb{E}}_{2}, \delta, R_{2}}\left(F_{y}(X), F_{y}\left(Y_{k}\right)\right) \leq N d_{\mathbb{F}_{1}, \mathbb{E}_{1}, \delta, R_{1}}\left(X, Y_{k}\right)
$$

Note that, by Proposition 2.13, we have

$$
d_{\mathbb{F}_{2}(y), \widetilde{\mathbb{E}}_{2}, \delta, R_{2}}\left(F_{y}(X), F_{y}\left(Y_{k}\right)\right) \stackrel{\text { law }}{=} d_{\mathrm{e}^{-\left(2+\gamma^{2} / 2\right) \log \left|\left(F_{y}^{-1}\right)^{\prime}\right|} \mu_{h_{\mathbb{F}_{1}}} \circ F_{y}^{-1}, \widetilde{\mathbb{E}}_{2}, \delta, R_{2}}\left(F_{y}(X), F_{y}\left(Y_{k}\right)\right) .
$$

On the other hand, we have

$$
d_{\mathbb{F}_{2}(y), \widetilde{\mathbb{E}}_{2}, \delta, R_{2}}\left(F_{y}(X), F_{y}\left(Y_{k}\right)\right) \geq d_{\mathbb{F}_{2}(y), \widetilde{\mathbb{E}}_{2}, \delta, R_{2}}\left(F_{y}(X), F_{y}\left(Y_{k}\right)\right) \geq d_{\mathbb{F}_{2}(y), \widetilde{\mathbb{E}}_{2}, \delta, R_{2}}\left(\widetilde{\mathbb{E}}_{2} \cap L_{\mathbb{R}_{2}}, \widetilde{\mathbb{E}}_{2} \cap \mathbf{R}_{\mathbb{R}_{2}}\right),
$$


and on the event $E^{\prime}=\left\{\max _{z \in \widetilde{\mathbb{E}}_{2}^{\left(R_{2}\right)}} h_{\mathbb{R}_{2}^{*}: \mathbb{F}_{2}(y)}(z) \leq 0\right\}$ (which is independent of $d_{\mathbb{F}_{2}(y), \widetilde{\mathbb{E}}_{2}, \delta, R_{2}}\left(F_{y}(X), F_{y}\left(Y_{k}\right)\right)$ ) we have that

$$
d_{\mathbb{F}_{2}(y), \widetilde{\mathbb{E}}_{2}, \delta, R_{2}}\left(\widetilde{\mathbb{E}}_{2} \cap \mathrm{L}_{\mathbb{R}_{2}}, \widetilde{\mathbb{E}}_{2} \cap \mathrm{R}_{\mathbb{R}_{2}}\right) \geq d_{\mathbb{R}_{2}^{*}, \widetilde{\mathbb{E}}_{2}, \delta, R_{2}}\left(\widetilde{\mathbb{E}}_{2} \cap \mathrm{L}_{\mathbb{R}_{2}}, \widetilde{\mathbb{E}}_{2} \cap \mathrm{R}_{\mathbb{R}_{2}}\right) .
$$

This implies that, if we define $q_{* *}=\mathbf{P}\left[E^{\prime}\right]$ (which again is strictly positive since $\operatorname{dist}_{\mathrm{E}}\left(\widetilde{\mathbb{E}}_{2}^{\left(R_{2}\right)}, \partial \mathbb{F}_{2}(y)\right)>0$ ), then for any $v>0$,

$$
\mathbf{P}\left(d_{\mathbb{R}_{2}^{*}, \widetilde{\mathbb{E}}_{2}, \delta, R_{2}}\left(\widetilde{\mathbb{E}}_{2} \cap \mathrm{L}_{\mathbb{R}_{2}}, \widetilde{\mathbb{E}}_{2} \cap \mathrm{R}_{\mathbb{R}_{2}}\right) \leq v\right) \geq q_{* *} \mathbf{P}\left(d_{\mathbb{F}_{2}(y), \widetilde{\mathbb{E}}_{2}, \delta, R_{2}}\left(F_{y}(X), F_{y}\left(Y_{k}\right)\right) \leq v\right) .
$$

We can also write, using (2.26) and then (2.23),

$$
d_{\mathbb{R}_{2}^{*}, \widetilde{\mathbb{E}}_{2}, \delta, R_{2}}\left(\widetilde{\mathbb{E}}_{2} \cap \mathrm{L}_{\mathbb{R}_{2}}, \widetilde{\mathbb{E}}_{2} \cap \mathrm{R}_{\mathbb{R}_{2}}\right) \geq d_{\mathbb{R}_{2}, \delta, R_{2}}\left(\widetilde{\mathbb{E}}_{2} \cap \mathrm{L}_{\mathbb{R}_{2}}, \widetilde{\mathbb{E}}_{2} \cap \mathrm{R}_{\mathbb{R}_{2}}\right) \geq d_{\mathbb{R}_{2}, \delta, R_{2}}(\mathrm{~L}, \mathrm{R}) \geq d_{\mathbb{R}_{2}, \delta}(\mathrm{L}, \mathrm{R}) .
$$

Therefore, we have

$$
\begin{aligned}
& \mathbf{P}\left(d_{\mathbb{R}_{2}, \delta}(\mathrm{L}, \mathrm{R}) \leq C N w\right) \\
& \quad \geq \mathbf{P}\left(d_{\mathbb{R}_{2}^{*}, \widetilde{\mathbb{E}}_{2}, \delta, R_{2}}\left(\widetilde{\mathbb{E}}_{2} \cap \mathrm{L}_{\mathbb{R}_{2}}, \widetilde{\mathbb{E}}_{2} \cap \mathrm{R}_{\mathbb{R}_{2}}\right) \leq C N w\right) \geq q_{* *} \mathbf{P}\left(d_{\mathbb{F}_{2}(y), \widetilde{\mathbb{E}}_{2}, \delta, R_{2}}\left(F_{y}(X), F_{y}\left(Y_{k}\right)\right) \leq C N w\right) \\
& \quad \geq q_{* *} \mathbf{P}\left(d_{\mathrm{e}^{-\left(2+\gamma^{2} / 2\right) \log \left|\left(F_{y}^{-1}\right)^{\prime}\right|} \mu_{h_{\mathbb{E}_{1}}} \circ F_{y}^{-1, \widetilde{\mathbb{E}}_{2}, \delta, R_{2}}}\left(F_{y}(X), F_{y}\left(Y_{k}\right)\right) \leq C N w\right) \geq q_{* *} \mathbf{P}\left(d_{\mathbb{F}_{1}, \mathbb{E}_{1}, \delta, R_{1}}\left(X, Y_{k}\right) \leq C w\right) \\
& \quad \geq \frac{q_{* *} q_{*} q_{0}}{3 Q},
\end{aligned}
$$

where the first inequality is by (3.26), the second is by (3.25), the third is by (3.24), the fourth is by (3.23) and (2.29) (applied with $\alpha=\mathrm{e}^{\left(2+\gamma^{2} / 2\right) \log \left|\left(F_{y}^{-1}\right)^{\prime}\right|} \vee 1$, which we fold into the constant $C$ as it is bounded by (3.20)), and the last is by (3.22). This implies (3.18) with appropriately chosen constants.

\section{Percolation arguments}

In this section we prove concentration for crossing distances at a given scale, as well as relationships between crossing distances at different scales, using percolation-type arguments. Recall the definition (2.19) of $d \ldots(\min ; a)$.

Proposition 4.1. There is a $p_{0}>0$ so that for every $\theta>2$, there is a constant $C<\infty$ so that the following holds. For any $a>0$, any box $\mathbb{R}$ with $\mathrm{AR}(\mathbb{R}) \in[1 / 3,3]$, any $R>0$, and any $K \in[1, \infty)$, if we define

$$
\begin{aligned}
& S=\operatorname{diam}_{E}(\mathbb{R}), \\
& \omega=\frac{2 \gamma \theta}{4+\gamma^{2}},
\end{aligned}
$$

then we have that

$$
\mathbf{P}\left(d_{\mathbb{R}, \delta, R}(\min ; a) \leq \Theta_{\mathbb{B}\left(K^{-1-\omega} S\right), \delta, K^{-\omega}{ }_{R}}^{\text {easy }}\left(p_{0}\right)\right) \leq C\left(K^{2-\theta^{2} / 2}+\mathrm{e}^{-a K / C}\right) .
$$

If we further assume that $R \leq K^{-1} S$, then we have that

$$
\mathbf{P}\left(d_{\mathbb{R}, \delta, R}(\min ; a) \leq \frac{a K}{C} \Theta_{\mathbb{B}\left(K^{-1-\omega}\right), \delta, K^{-\omega} R}^{\mathrm{easy}}\left(p_{0}\right)\right) \leq C\left(K^{2-\theta^{2} / 2}+\mathrm{e}^{-a K / C}\right) .
$$


We will prove Proposition 4.1 in Subsection 4.3. The constant $p_{0}$ will remain fixed throughout the remainder of the paper. Note that if $a$ is treated as a fixed constant, as it often will be in the sequel, then the second terms of the right-hand sides of (4.2) and (4.3) can be ignored.

Proposition 4.2. We have constants $p_{1}<1$ and $C<\infty$ so that the following holds. Let $\mathbb{R}$ be a box with $\operatorname{AR}(\mathbb{R}) \in[1 / 3,3]$, let $S=\operatorname{diam}_{\mathbb{E}}(\mathbb{R})$, and let $K \in[1, \infty)$. Then we have, with $\eta$ as in (2.12), that

$$
\mathbf{P}\left(d_{\mathbb{R}, \delta, R}(\mathrm{~L}, \mathrm{R}) \geq \mathrm{e}^{K^{7 / 8}} \Theta_{\mathbb{B}\left(K^{\eta-1} S\right), \delta, K^{\eta} R}^{\text {hard }}\left(p_{1}\right)\right) \leq C \mathrm{e}^{-K / C} .
$$

Moreover, for every $p>0$ there is a $K_{0}<\infty$ so that if $K \geq K_{0}$, then for every $\mathbb{R}$ with $\operatorname{AR}(\mathbb{R}) \in[1 / 3,3]$, and setting $S=\operatorname{diam}_{\mathrm{E}}(\mathbb{R})$, we have

$$
\mathbf{P}\left(d_{\mathbb{R}, \delta, R}(\mathrm{~L}, \mathrm{R}) \geq K^{C} \Theta_{\mathbb{B}\left(K^{\eta-1} S\right), \delta, K^{\eta} R}^{\text {hard }}\left(p_{1}\right)\right) \leq p .
$$

We will prove Proposition 4.2 in Subsection 4.4. Like $p_{0}$, the constant $p_{1}$ will remain fixed throughout the remainder of the paper.

Before establishing some important applications and consequences of Propositions 4.1 and 4.2, we will set up some notation that we use to express the ratios of different quantiles to each other. Then, in Subsections 4.1 and 4.2, we will use the notation to express bounds on quantiles, moments, and tails of certain crossing distances, using Theorem 3.4 and Propositions 4.1 and 4.2. Later, in Section 5, we will show that these ratios are not too large by induction on the scale.

Definition 4.3. For $U \in(0, \infty)$, define

$$
\chi_{U}=\frac{\Theta_{\mathbb{B}(U)}^{\text {hard }}\left(p_{1}\right)}{\Theta_{\mathbb{B}(U)}^{\text {easy }}\left(p_{0}\right)}
$$

and

$$
\bar{\chi}_{U}=\sup _{V \in(0, U]} \chi_{V}
$$

Further define $\mathcal{S}_{\chi}=\left\{U>0: \chi_{U} \leq \chi\right\}$.

By Theorem 3.4 and Lemma A.1, we have a constant $C<\infty$ so that

$$
\chi_{U} \leq C \exp \left\{C \sqrt{\operatorname{Var}\left(\log d_{\mathbb{B}(U)}(\text { hard })\right)}\right\} .
$$

Thus, our strategy to bound $\chi_{U}$ will be to inductively bound $\operatorname{Var}\left(\log d_{\mathbb{B}(U)}(\right.$ hard $\left.)\right)$. Also, it is proved in [12, (74)] that there is a constant $C$ so that

$$
\bar{\chi}_{U} \leq C \mathrm{e}^{(\log (U+1))^{0.95}}
$$

for all $U$. This will be important for the base case of our induction.

\subsection{Quantile relationships}

In this section, we show how Subsections 4.1 and 4.2 allow us to prove upper and lower bounds on ratios between quantiles of crossing distances at different scales. 


\subsubsection{Easy crossing quantile ratios}

Here we prove the following proposition establishing at least power-law growth of easy crossing quantiles.

Proposition 4.4. There are constants $T_{0} \geq 0$ and $c>0$ so that for every $S \geq T_{0}$ and $K \geq 1$, we have

$$
\Theta_{\mathbb{B}(K S)}^{\text {easy }}\left(p_{0}\right) \geq c K^{c} \Theta_{\mathbb{B}(S)}^{\text {easy }}\left(p_{0}\right) .
$$

To prove Proposition 4.4, we will require the following lemma.

Lemma 4.5. We have constants $c>0$ and $C<\infty$ so that for any $M \geq 1$ and any $S>0$, we have that

$$
\Theta_{\mathbb{B}(M S)}^{\text {easy }}\left(p_{0}\right) \geq c M^{1 / 2} \Theta_{\mathbb{B}(S)}^{\text {easy }}\left(p_{0}\right)-C M^{3 / 2}
$$

Proof. Choose $\theta=\frac{4+\gamma^{2}}{2 \gamma}>2$. Note that with this choice of $\theta$ we have $\omega=1$ in the statement of Proposition 4.1. If $K$ is large enough so that $C K^{2-\theta^{2} / 2} \leq p_{0}$, then we have that

$$
\Theta_{\mathbb{B}\left(K^{2} S\right), 1, \sqrt{5} K S}^{\text {easy }}\left(p_{0}\right) \geq \Theta_{\mathbb{B}\left(K^{2} S\right), 1, \sqrt{5} K S}^{\text {easy }}\left(C K^{2-\theta^{2} / 2}\right) \geq \frac{K}{C} \Theta_{\mathbb{B}(S), 1, \sqrt{5} S}^{\text {easy }}\left(p_{0}\right)=\frac{K}{C} \Theta_{\mathbb{B}(S)}^{\text {easy }}\left(p_{0}\right)
$$

by (4.3) of Proposition 4.1. On the other hand, by (2.31) of Lemma 2.23, we have a constant $C$ so that

$$
\Theta_{\mathbb{B}\left(K^{2} S\right), 1, \sqrt{5} K S}^{\text {easy }}\left(p_{0}\right) \leq \Theta_{\mathbb{B}\left(K^{2} S\right)}^{\text {easy }}\left(p_{0}\right)+C K^{3} .
$$

Combining this with (4.10), we have

$$
\Theta_{\mathbb{B}\left(K^{2} S\right)}^{\text {easy }}\left(p_{0}\right)+C K^{3} \geq \frac{K}{C} \Theta_{\mathbb{B}(S)}^{\text {easy }}\left(p_{0}\right),
$$

which proves (4.9) when we take $M=K^{2}$.

Now we can prove Proposition 4.4 by induction.

Proof of Proposition 4.4. By Lemma 4.5, we can choose $M>1$ so that $\Theta_{\mathbb{B}(M S)}^{\text {easy }}\left(p_{0}\right) \geq 2 \Theta_{\mathbb{B}(M S)}^{\text {easy }}\left(p_{0}\right)-C$ for some constant $C<\infty$ (distinct, of course, from those in (4.9)). By induction, we have

$$
\Theta_{\mathbb{B}\left(M^{k} S\right)}^{\text {easy }}\left(p_{0}\right) \geq 2^{k} \Theta_{\mathbb{B}(S)}^{\text {easy }}\left(p_{0}\right)-\sum_{j=0}^{k-1} 2^{j} C \geq 2^{k}\left(\Theta_{\mathbb{B}(S)}^{\text {easy }}\left(p_{0}\right)-C\right) .
$$

Now let $k=\left\lfloor\log _{M} K\right\rfloor$. Then we have, by (2.40) and (4.11), that

$$
\Theta_{\mathbb{B}(K S)}^{\text {easy }}\left(p_{0}\right) \geq \Theta_{\mathbb{B}\left(M^{k} S\right)}^{\text {easy }}\left(p_{0}\right) \geq 2^{k}\left(\Theta_{\mathbb{B}(S)}^{\text {easy }}\left(p_{0}\right)-C\right) \geq \frac{1}{2} K^{\log _{M} 2}\left(\Theta_{\mathbb{B}(S)}^{\text {easy }}\left(p_{0}\right)-C\right) .
$$

Now by Lemma 2.37, there is a $T_{0}$ so that if $S \geq T_{0}$, then $\Theta_{\mathbb{B}(S)}^{\text {easy }}\left(p_{0}\right) \geq 2 C$. Therefore,

$$
\Theta_{\mathbb{B}(K S)}^{\text {easy }}\left(p_{0}\right) \geq \frac{1}{2} K^{\log _{M} 2} \Theta_{\mathbb{B}(S)}^{\text {easy }}\left(p_{0}\right),
$$

which is (4.8) with the appropriate choice of $c$. 


\subsubsection{Hard crossing quantile ratios}

Here we show that the hard-crossing quantiles can grow at most like a power of the scale. We will in fact use only (4.13) of the following proposition in the sequel, but we include (4.12) as well for completeness.

Proposition 4.6. For every $p \in(0,1)$ there is a $K_{1}<\infty$ and a constant $C<\infty$ so that if $K \geq K_{1}$, then we have

$$
\Theta_{\mathbb{B}(K S)}^{\text {hard }}(p) \leq K^{C} \Theta_{\mathbb{B}(S)}^{\text {hard }}\left(p_{1}\right)
$$

and

$$
\Theta_{\mathbb{B}(K S)}^{\text {easy }}(p) \leq K^{C} \Theta_{\mathbb{B}(S)}^{\text {hard }}\left(p_{1}\right) .
$$

Proof. This follows from (4.5) of Proposition 4.2, taking $K=K^{\eta-1}$.

\subsection{Tail and moment estimates}

In this section, we use the results of the percolation arguments (Propositions 4.1 and 4.2) to prove moment and tail estimates on crossing probabilities. Recall the definition of $\chi_{U}$ and $\bar{\chi}_{U}$ from Definition 4.3.

\subsubsection{Moment estimates for easy crossings}

Proposition 4.7. For any $A, Q>0$ there exists a constant $C=C(A, Q)<\infty$ so that if a $\in(0,1), \mathbb{R}$ is a rectangle with $\mathrm{AR}(\mathbb{R}) \in[1 / 3,3]$, and $S=\operatorname{diam}_{\mathrm{E}}(\mathbb{R})$, then we have

$$
\mathbf{E}\left[d_{\mathbb{R}}(\min ; a)^{-A}\right] \leq C\left(1+a^{-C}\right) \bar{\chi}_{Q^{-1} S}^{A} \Theta_{\mathbb{B}(S)}^{\text {easy }}\left(p_{0}\right)^{-A} .
$$

Proof. We first recall the simple formula

$$
\mathbf{E}\left[d_{\mathbb{R}}(\min ; a)^{-A}\right]=\int_{0}^{\infty} \mathbf{P}\left[d_{\mathbb{R}}(\min ; a) \leq u^{-1 / A}\right] \mathrm{d} u .
$$

We have by (4.2) of Proposition 4.1 that, if we define

$$
\omega(\theta)=\frac{2 \gamma \theta}{4+\gamma^{2}}
$$

as in (4.1), then

$$
\mathbf{P}\left(d_{\mathbb{R}}(\min ; a) \leq \Theta_{\mathbb{B}\left(K^{-1-\omega(\theta)} S\right), 1, K^{-\omega(\theta)} S}^{\text {easy }}\left(p_{0}\right)\right) \leq C\left(K^{2-\theta^{2} / 2}+\mathrm{e}^{-a K / C}\right) .
$$

But on the other hand, we have, as long as $K^{1+\omega(\theta)} \geq K_{1}$ from Proposition 4.6,

$$
\begin{aligned}
\Theta_{\mathbb{B}\left(K^{-1-\omega(\theta)} S\right), 1, K^{-\omega(\theta)} \sqrt{5} S}^{\text {easy }}\left(p_{0}\right)=\Theta_{\mathbb{B}\left(K^{-1-\omega(\theta)} S\right)}^{\text {easy }}\left(p_{0}\right) & \geq \chi_{K^{-1-\omega(\theta)} S}^{-1} \Theta_{\mathbb{B}\left(K^{-1-\omega(\theta)} S\right)}^{\text {hard }}\left(p_{1}\right) \\
& \geq C K^{-C(1+\omega(\theta))} \chi_{K^{-1-\omega(\theta)} S}^{-1} \Theta_{\mathbb{B}(S)}^{\text {easy }}\left(p_{1}\right),
\end{aligned}
$$

where the equality is by (2.24), the first inequality is by the definition of $\chi_{K^{-1-\omega(\theta)} S}$, and the second inequality is by (4.13) of Proposition 4.6. Applying this to (4.16) yields

$$
\mathbf{P}\left(d_{\mathbb{R}}(\min ; a) \leq K^{-C(1+\omega(\theta))} \chi_{K^{-1-\omega(\theta)} S}^{-1} \Theta_{\mathbb{B}(S)}^{\mathrm{hard}}\left(p_{1}\right)\right) \leq C\left(K^{2-\theta^{2} / 2}+\mathrm{e}^{-a K / C}\right) .
$$

This implies that

$$
\mathbf{P}\left(\left(\frac{d_{\mathbb{R}}(\min ; a) \chi_{K^{-1-\omega(\theta)} S}}{\Theta_{\mathbb{B}(S)}^{\operatorname{easy}}\left(p_{1}\right)}\right)^{-A} \geq K^{A C(1+\omega(\theta))}\right) \leq C\left(K^{2-\theta^{2} / 2}+\mathrm{e}^{-a K / C}\right) .
$$




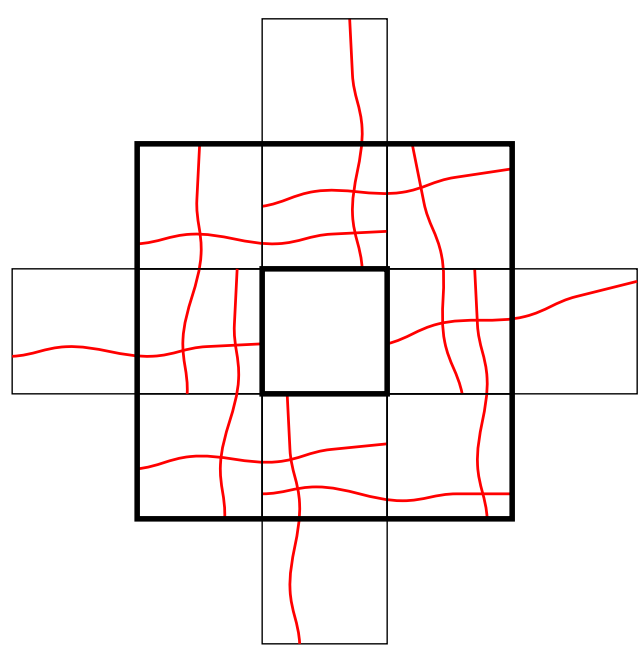

(a) Creating an annular crossing from hard crossings of rectangles.

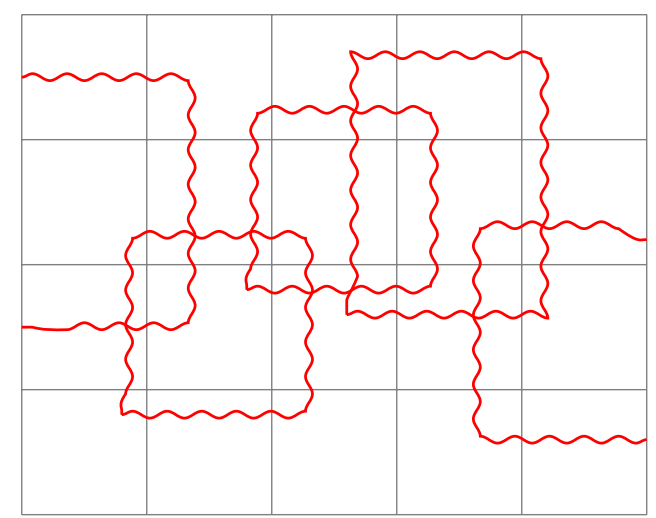

(b) Assembling annuli into a crossing.

Figure 4.1

Putting $u=K^{A C(1+\omega(\theta))}$ yields, for each $Q<\infty$, a $u_{0}<\infty$ so that if $u \geq u_{0}$ then

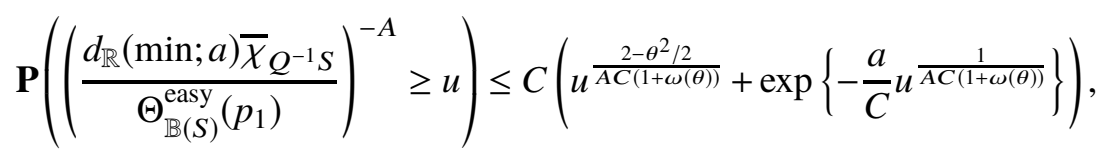

and thus

$$
\mathbf{E}\left[\left(\frac{d_{\mathbb{R}}(\min ; a)}{\Theta_{\mathbb{B}(S)}^{\text {easy }}\left(p_{1}\right)}\right)^{-A}\right] \leq C \bar{\chi}_{Q^{-1} S}^{A}\left(u_{0}+\int_{u_{0}}^{\infty} 1 \vee u^{\frac{2-\theta^{2} / 2}{A C(1+\omega(\theta))}} \mathrm{d} u+\int_{0}^{\infty} \exp \left\{-\frac{a}{C} u^{\frac{1}{A C(1+\omega(\theta))}}\right\} \mathrm{d} u\right) .
$$

Now $\theta$ can be chosen large enough so that $\frac{2-\theta^{2} / 2}{A C(1+\omega(\theta))}<1$ (recalling the definition (4.15) of $\omega(\theta)$ ) and hence the first integral of the last expression is finite. On the other hand, by a change of variables, the second integral is a constant times $a^{-A C(1+\omega(\theta))}$. This implies (4.14).

\subsubsection{Tail and moment estimates for hard crossings}

In this section we establish estimates for the tails of hard-crossing distances and for moments of crossings of annuli. We state our bound for hard crossings in terms of a tail bound rather than in terms of moments because in the proof of Proposition 6.4 below, we will need an explicit superpolynomial concentration bound for hard-crossing distances.

Proposition 4.8. There is a constant $C<\infty$ so that for any $S, \delta, R, \theta>0$ we have

$$
\mathbf{P}\left(d_{\mathbb{B}(S), \delta, R}(\text { hard }) \geq(1+\theta) \Theta_{\mathbb{B}(S), \delta, R}^{\mathrm{hard}}\left(p_{1}\right)\right) \leq C \mathrm{e}^{-(\log (1+\theta))^{8 / 7} / C} .
$$

Proof. This follows from (4.4) of Proposition 4.2, taking $K=(\log (1+\theta))^{8 / 7}$, and Proposition 2.36.

Corollary 4.9. For any $B \geq 0$ we have a constant $C<\infty$ so that the following holds. Let $\delta, R>0$. Suppose that $\mathbb{B}$ is an $S \times S$ square, $\mathbb{R}$ is a rectangle so that either $3 \mathbb{B} \backslash \mathbb{B} \subset \mathbb{R}$ or $(3 \mathbb{B} \backslash \mathbb{B}) \cap \partial \mathbb{R}$ has exactly two connected components, and $\mathbb{A}=(3 \mathbb{B} \backslash \mathbb{B}) \cap \mathbb{R}$. Then we have, recalling the definition (2.20) of $d \ldots$ (around), that

$$
\mathbf{E}\left[d_{(3 \mathbb{B})^{*}, \mathbb{A}, \delta, R}(\text { around })^{B}\right] \leq C \Theta_{\mathbb{B}(S), \delta, R}^{\mathrm{hard}}\left(p_{1}\right)^{B} .
$$


Proof. The construction illustrated in Figure 4.1a shows how to build an annular circuit out of hard crossings of twelve rectangles. Thus, union-bounding Proposition 4.8 over these twelve rectangles, and using (2.42) (of Proposition 2.38) and Corollary 2.8 to control the effect of the coarse field on these rectangles, we have

$$
\mathbf{P}\left(d_{(3 \mathbb{B})^{*}, \mathbb{A}, \delta, R}(\text { around }) \geq \theta \Theta_{\mathbb{B}(S), \delta, R}^{\mathrm{hard}}\left(p_{1}\right)\right) \leq C \mathrm{e}^{-(\log (\theta+1))^{8 / 7} / C} .
$$

Then we have

$\mathbf{E}\left[\left(\frac{d_{(3 \mathbb{B})^{*}, \mathbb{A}, \delta, R}(\text { around })}{\Theta_{\mathbb{B}(S), \delta, R}^{\text {hard }}\left(p_{1}\right)}\right)^{B}\right]=\int_{0}^{\infty} \mathbf{P}\left(\frac{d_{(3 \mathbb{B})^{*}, \mathbb{A}, \delta, R}(\text { around })}{\Theta_{\mathbb{B}(S), \delta, R}^{\text {hard }}\left(p_{1}\right)} \geq \theta^{1 / B}\right) \mathrm{d} \theta \leq C \int_{0}^{\infty} \mathrm{e}^{-(\log (1+\theta))^{8 / 7} /\left(C B^{8 / 7}\right)} \mathrm{d} \theta<C$,

where as usual, the constant $C$ has been allowed to change from step to step. This proves (4.17).

\subsection{Proof of Proposition 4.1}

In this section, we prove Proposition 4.1. We do this using a percolation argument, relying on the fact that a path between two macroscopically separated points must make many easy crossings of smaller boxes. The geometry used in the arguments of this subsection is essentially identical to that of [8, Section 4].

Proof of Proposition 4.1. We claim that there are constants $c>0$ and $C<\infty$, independent of $a$, so that, for every $K \geq C$, there is a collection $C_{K}$ of $K^{-1} S \times 2 K^{-1} S$ and $2 K^{-1} S \times K^{-1} S$ subboxes of $\mathbb{R}$ and a set $\mathscr{C}_{a, K}$ of subsets of $C_{K}$ so that the following properties hold.

1. $\left|C_{K}\right| \leq C K^{2}$.

2. All elements $\mathcal{D} \subset \mathscr{C}_{a, K}$ have the same cardinality $N \in[c K a, C K a]$.

3. $\left|\mathscr{C}_{a, K}\right| \leq C^{N}$. (As we describe below, this is a consequence of the fact that each element of $\mathscr{C}_{a, K}$ is a walk of length $N$ on a bounded-degree graph.)

4. For each $\mathbb{C}_{1}, \mathbb{C}_{2} \in \mathcal{D} \in \mathscr{C}_{a, K}$, we have $\operatorname{dist}_{\mathbb{E}}\left(\mathbb{C}_{1}^{*}, \mathbb{C}_{2}^{*}\right)>0$.

5. Whenever $x, y \in \mathbb{R}$ and $|x-y| \geq a \operatorname{diam}_{\mathbb{E}}(\mathbb{R})$, if $\pi$ is any path between $x$ and $y$ then we have a $\mathcal{D}(\pi) \in$ $\mathscr{C}_{a, K}$ so that $\pi$ includes an easy crossing of $\mathbb{C}$ for each $\mathbb{C} \in \mathcal{D}(\pi)$.

These collections can be constructed as follows. First, construct at most $C K^{2}$ annuli which are all congruent to $\mathbb{B}\left(3 K^{-1} S, 3 K^{-1} S\right) \backslash \mathbb{B}\left(K^{-1} S, K^{-1} S\right)$, such that the regions enclosed by the inner boundaries of the annuli cover all of $\mathbb{R}$. Cover each annulus with eight boxes, each having dimensions $K^{-1} S \times 2 K^{-1} S$ or $2 K^{-1} S \times$ $K^{-1} S$. Let $C_{K}$ be the set of all of these boxes, translated by a minimal amount so that each lies completely inside $\mathbb{R}$. Now any path that starts in the region enclosed by the inner boundary of an annulus, and then travels outside of the annulus, must make an easy crossing of one of the eight boxes associated to that annulus. (Figure 4.2.) Therefore, if a path travels a distance $a \operatorname{diam}_{\mathrm{E}}(\mathbb{R})$, it must cross at least $c a K$ of the boxes in $C_{K}$. From these boxes we can extract a subset of boxes $\left\{\mathbb{C}_{i}\right\}$ such that $\left\{\mathbb{C}_{i}^{*}\right\}$ (recall that $\left.\mathbb{C}_{i}^{*}=\mathbb{C}_{i}^{\left(2 \operatorname{diam}_{\mathrm{E}} \mathbb{C}_{i}\right)}\right)$ is a pairwise-disjoint family by a greedy algorithm. There still must be at least $c a K$ elements in this subset, perhaps with a smaller constant $c$, and thus we can truncate all of these subsets so they all have the same size $N \geq c K a$. Call the subset associated to the path $\mathcal{D}(\pi)$ and let $\mathscr{C}_{a, K}=\{\mathcal{D}(\pi) \mid$ $x, y \in \mathbb{R},|x-y| \geq a \operatorname{diam}_{\mathbb{E}}(\mathbb{R})$, and $\pi$ is a path between $x$ and $\left.y\right\}$. Now the cardinality of $\mathscr{C}_{a, K}$ can be at most $C^{N}$, since successive elements of $\mathcal{D}(\pi)$ can only be a bounded distance from each other, and so $\mathcal{D}(\pi)$ is a walk on a bounded-degree graph of size $N$. 


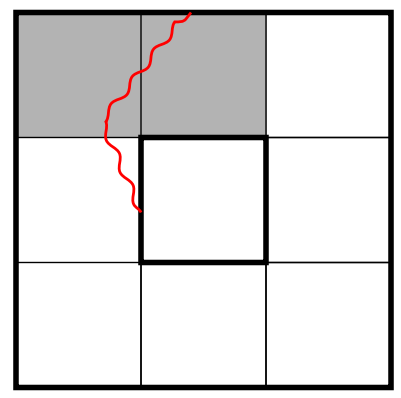

Figure 4.2: A path from the inside boundary to the outside boundary of an annulus must make an easy crossing of one of the eight rectangles that cover the boundary of the annulus.

Now let $x, y \in \mathbb{R}$ be such that $|x-y| \geq a \operatorname{diam}_{\mathrm{E}}(\mathbb{R})$ and let $\pi$ be a $\left(\mu_{\mathbb{R}^{*}}, \mathbb{R}, \delta, R\right)$-geodesic (in the sense of Definition 2.22) between $x$ and $y$. Therefore, there is a $\mathcal{D} \in \mathscr{C}_{a, K}$ so that $\pi$ includes an easy-crossing of every $\mathbb{C} \in \mathcal{D}$. Let

$$
F=\min _{\mathbb{C} \in \mathcal{C}_{K}} \min _{x \in \mathbb{C}^{\circ}} h_{\mathbb{R}^{*}: \mathbb{C}^{*}}(x) .
$$

If $R \leq K^{-1} S$, then we have

$$
d_{\mathbb{R}, \delta, R}(x, y) \geq \sum_{\mathbb{C} \in \mathcal{D}} d_{\mathbb{R}^{*}, \mathbb{C}, \delta, R}(\text { easy }) \geq \sum_{\mathbb{C} \in \mathcal{D}} d_{\mathbb{C}, \mathrm{e}^{\gamma F}, R \wedge\left(\sqrt{5} K^{-1} S\right)}(\text { easy })
$$

where the first inequality is by Proposition 2.29 (in which the hypothesis that the $\mathbb{C}_{i} \mathrm{~s}$ are separated by at least $R$ is satisfied by the condition on $R$ and property 4 above), and the second inequality is by Proposition 2.38. On the other hand, if we do not assume that $R \leq K^{-1} S$, then we still have that

$$
d_{\mathbb{R}, \delta, R}(x, y) \geq \max _{\mathbb{C} \in \mathcal{D}} d_{\mathbb{R}^{*}, \mathbb{C}, \delta, R}\left(\text { easy) } \geq \max _{\mathbb{C} \in \mathcal{D}} d_{\mathbb{R}^{*}, \mathbb{C}, \delta, R \wedge\left(\sqrt{5} K^{-1} S\right)}\left(\text { easy) } \geq \max _{\mathbb{C} \in \mathcal{D}} d_{\mathbb{C}, \mathrm{e}^{\gamma F} \delta, R \wedge\left(\sqrt{5} K^{-1} S\right)}(\text { easy), }\right.\right.
$$

where the first inequality is by Proposition 2.28, the second is by (2.24), and the third is by Proposition 2.38.

Recall $N$ from property 2 above and let $u>0$ (to be fixed in (4.22) below). Under the assumption that $R \leq K^{-1} S$, (4.18) implies that on the event that $d_{\mathbb{R}, \delta, R}(\max ; a) \leq N u / 2$, the set $\mathcal{D}(\pi)$ (defined in property 5

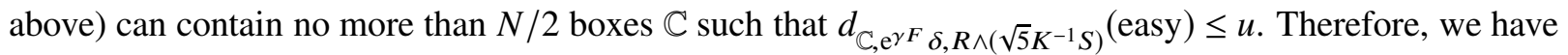

$$
\begin{aligned}
& \mathbf{P} {\left[d_{\mathbb{R}, \delta, R}(\min ; a) \leq N u / 2\right] } \\
& \leq \mathbf{P}\left[\text { there exists a } \mathcal{D} \in \mathscr{C}_{a, K} \text { such that } \#\left\{\mathbb{C} \in \mathcal{D}: d_{\mathbb{C}, \mathrm{e}^{\gamma F} \delta, R \wedge\left(\sqrt{5} K^{-1} S\right)}(\text { easy }) \leq u\right\} \geq N / 2\right] \\
& \leq \mathbf{P}\left[\text { there exists a } \mathcal{D} \in \mathscr{C}_{a, K} \text { such that } \#\left\{\mathbb{C} \in \mathcal{D}: d_{\mathbb{C}, K^{\gamma \theta} \delta, R \wedge\left(\sqrt{5} K^{-1} S\right)}(\text { easy }) \leq u\right\} \geq N / 2\right] \\
& \quad \quad+\mathbf{P}[F \leq-\theta \log K] \\
& \quad: \Xi_{1}+\Xi_{2},
\end{aligned}
$$

where the second inequality is by a union bound and $\Xi_{1}$ and $\Xi_{2}$ are the first and second terms of the secondto-last expression, respectively. On the other hand, if we do not assume that $R \leq K^{-1} S$, then we still have, by (4.19), that

$$
\begin{aligned}
& \mathbf{P}\left[d_{\mathbb{R}, \delta, R}(\min ; a) \leq u\right] \leq \mathbf{P}\left[\text { there exists a } \mathcal{D} \in \mathscr{C}_{a, K} \text { such that } \max _{\mathbb{C} \in \mathcal{D}} d_{\mathbb{C}, \mathrm{e}^{\gamma F} \delta, R \wedge\left(\sqrt{5} K^{-1} S\right)}(\text { easy }) \leq u\right] \\
& \leq \mathbf{P}\left[\text { there exists a } \mathcal{D} \in \mathscr{C}_{a, K} \text { such that } \max _{\mathbb{C} \in \mathcal{D}} d_{\mathbb{C}, K^{\gamma \theta} \delta, R \wedge\left(\sqrt{5} K^{-1} S\right)}(\text { easy }) \leq u\right]+\mathbf{P}[F \leq-\theta \log K] \leq \Xi_{1}+\Xi_{2},
\end{aligned}
$$


where the last inequality is because, if $\max _{\mathbb{C} \in \mathcal{D}} d_{\mathbb{C}, K^{\gamma \theta} \delta, R \wedge\left(\sqrt{5} K^{-1} S\right)}$ (easy) $\leq u$, then in particular

$$
\#\left\{\mathbb{C} \in \mathcal{D}: d_{\mathbb{C}, K^{\gamma \theta} \delta, R \wedge\left(\sqrt{5} K^{-1} S\right)}(\text { easy }) \leq u\right\}=|\mathcal{D}|=N \geq N / 2 .
$$

Now define, for some $p_{0} \in(0,1)$ to be chosen later,

$$
u=\Theta_{\mathbb{B}\left(K^{-1} S\right), K^{\gamma \theta} \delta, R \wedge\left(\sqrt{5} K^{-1} S\right)}^{\text {easy }}\left(p_{0}\right)=\Theta_{\mathbb{B}\left(K^{-\omega-1} S\right), \delta, K^{-\omega}\left(R \wedge\left(\sqrt{5} K^{-1} S\right)\right)}^{\text {easy }}\left(p_{0}\right)=\Theta_{\mathbb{B}\left(K^{-\omega-1} S\right), \delta, K^{-\omega} R}^{\text {easy }}\left(p_{0}\right),
$$

where the second equality is by Proposition 2.35 and the second is by (2.24). (Recall that $\omega$ was defined in (4.1).) By Lemma A.2 and a union bound along with property 3 above, we have

$$
\Xi_{1} \leq\left|\mathscr{C}_{a, K}\right| p_{0}^{N / 2} \leq C^{K}\left(C p_{0}\right)^{N / 2} \leq C^{N} p_{0}^{N / 2}
$$

On the other hand, we have

$$
\Xi_{2} \leq C K^{2-\theta^{2} / 2}
$$

by (2.6) of Proposition 2.7. Plugging the last two bounds into (4.20) and (4.21), and noting (4.22), we obtain

$$
\begin{aligned}
\mathbf{P}\left[d_{\mathbb{R}, \delta, R}(\min ; a) \leq \frac{N}{2} \Theta_{\mathbb{B}\left(K^{-\omega-1} S\right), \delta, K^{-\omega} R}^{\text {easy }}\left(p_{0}\right)\right], \mathbf{P}\left[d_{\mathbb{R}, \delta, R}(\min ; a) \leq \Theta_{\mathbb{B}\left(K^{-\omega-1} S\right), \delta, K^{-\omega}{ }_{R}}^{\text {easy }}\left(p_{0}\right)\right] & \\
& \leq C^{N} p_{0}^{N / 2}+C K^{2-\theta^{2} / 2}
\end{aligned}
$$

Then we obtain (4.2) and (4.3) by taking $p_{0}$ so small that $C \sqrt{p_{0}}<1$ and recalling property 2 above.

\subsection{Proof of Proposition 4.2}

Although the statement of Proposition 4.2 is similar in spirit to [8, Proposition 6.1], here we need a better bound and thus use a more sophisticated percolation argument.

Proof of Proposition 4.2. We assume that $\mathrm{AR}(\mathbb{R}) \in\{1 / 3,3\}$ and that $K$ is an integer, the general case follows from Proposition 2.36 and a simple coarse-field bound. Choose an appropriate $L \in\{K / 3, K\}$ and divide $\mathbb{R}$ into a $K \times L$ grid of $S \times S$ subboxes, indexing them according to their position in the grid $\mathcal{G}_{K, L}=\{1, \ldots, K\} \times$ $\{1, \ldots, L\}$ as $\left(\mathbb{C}_{k, \ell}\right)_{(k, \ell) \in \mathcal{G}}$, with the following layout:

$$
\begin{array}{ccc}
\mathbb{C}_{1, L} & \cdots & \mathbb{C}_{K, L} \\
\vdots & \cdot \cdot & \vdots \\
\mathbb{C}_{1,1} & \cdots & \mathbb{C}_{K, 1}
\end{array}
$$

For each $(k, \ell) \in \mathcal{G}_{K, L}$, define $\mathbb{A}_{k, \ell}=\left(3 \mathbb{C}_{k, \ell} \backslash \mathbb{C}_{k, \ell}\right) \cap \mathbb{R}$, so $\mathbb{A}_{k, \ell}$ is an intersection of a square annulus with $\mathbb{R}$. Define $Q$ to be the set of paths $\omega=\left(\omega_{1}, \ldots, \omega_{J(\omega)}\right)$ so that $\omega_{j} \in \mathcal{G}_{K, L}$ for each $j$ and $\omega_{1}^{(1)}=1, \omega_{J(\omega)}^{(1)}=K$ (here the superscript (1) means to take the $x$-coordinate) and $\left|\omega_{j}-\omega_{j-1}\right|_{\infty} \leq 1$, where $|\cdot|_{\infty}$ denotes the $\ell^{\infty}$ norm. This is to say that the paths $\omega$ must cross from left to right in the grid $\mathcal{G}_{K, L}$ and must move as a chess king: one square at a time, either left, right, up, down, or diagonally. Then circuits around $\mathbb{A}_{\omega_{j}}, j=1, \ldots, J(\omega)$ can be joined together as illustrated in Figure $4.1 \mathrm{~b}$ to form a left-right crossing of $\mathbb{R}$, so we have

$$
d_{\mathbb{R}, \delta, R}(\mathrm{~L}, \mathrm{R}) \leq \min _{\omega \in Q} \sum_{j=1}^{J(\omega)} d_{\mathbb{R}^{*}, \mathbb{A}_{\omega_{j}}, \delta, R}(\text { around }) .
$$

(Recall the notation $d \ldots$ (around) defined in (2.20)-(2.21).) Define

$$
\mathcal{B}_{k, \ell}=\left\{\mathbb{B} \in \widetilde{\mathcal{B}}_{k, \ell}: \mathbb{B} \subset \mathbb{R}\right\},
$$


where $\widetilde{\mathcal{B}}_{k, \ell}$ comprises the eight unions of pairs of adjacent $\mathbb{C}_{k^{\prime}, \ell^{\prime}}$ s contained in $\mathbb{A}_{k, \ell}$, as well as the four unions of pairs $\left(\mathbb{C}_{k^{\prime}, \ell^{\prime}}, \mathbb{C}_{k^{\prime \prime}, \ell^{\prime \prime}}\right)$ such that $\mathbb{C}_{k^{\prime}, \ell^{\prime}}, \mathbb{C}_{k^{\prime \prime}, \ell^{\prime \prime}}$ are translates of $\mathbb{C}_{k, \ell}$ by one and two squares, respectively, either up, down, left, or right. That is, $\widetilde{\mathcal{B}}_{k, \ell}$ comprises twelve rectangles, and hard crossings of these rectangles can be joined together to create a circuit around $\mathbb{A}_{k, \ell}$ as shown in Figure 4.1a. This means that

$$
d_{\mathbb{R}^{*}, \mathbb{A}_{k, \ell}, \delta, R}(\text { around }) \leq \sum_{\mathbb{B} \in \mathcal{B}_{k, \ell}} d_{\mathbb{R}^{*}, \mathbb{B}, \delta, R}(\text { hard }) .
$$

Define

$$
F=\max _{(k, \ell) \in \mathcal{G}_{K, L}} \max _{\mathbb{B} \in \mathcal{B}_{k, \ell}} \max _{x \in \mathbb{B}^{\circ}} h_{\mathbb{R}^{*}: \mathbb{B}^{*}}(x) .
$$

By Corollary 2.8, we have, for any $v>1$, that

$$
\mathbf{P}\left[\mathrm{e}^{\gamma F-\gamma \theta_{0} \log K} \geq v\right] \leq C \mathrm{e}^{-\frac{(\log v)^{2}}{2 \gamma^{2} \log K}}
$$

(Recall that $\theta_{0}$ was fixed in (2.12).) Combining (4.23) and (4.24), we have

$$
d_{\mathbb{R}, \delta, R}(\mathrm{~L}, \mathrm{R}) \leq \min _{\omega \in Q} \sum_{j=1}^{J(\omega)} \sum_{\mathbb{B} \in \mathcal{B}_{k, \ell}} d_{\mathbb{R}^{*}, \mathbb{B}, \delta, R}(\text { hard }) \leq C \mathrm{e}^{\left(\gamma F-\gamma \theta_{0} \log K\right)^{+}} \min _{\omega \in Q} \sum_{j=1}^{J(\omega)} \sum_{\mathbb{B} \in \mathcal{B}_{k, \ell}} d_{\mathbb{B}, K^{-\gamma \theta_{0}} \delta, R}(\text { hard }) .
$$

Now we note that we can partition $\mathcal{G}$ into finitely many subsets $\mathcal{G}_{1}, \ldots, \mathcal{G}_{M}$, with $M$ an absolute constant not depending on $K$, so that

$$
\left\{\sum_{\mathbb{B} \in \mathcal{B}_{k, \ell}} d_{\mathbb{B}, K^{-\gamma \theta_{0}} \delta, R}(\text { hard }):(k, \ell) \in \mathcal{G}_{m}\right\}
$$

is a collection of independent random variables for each $1 \leq m \leq M$. Noting that $\left|\mathcal{B}_{k, \ell}\right| \leq 12$ for all $k$, $\ell$, we have by a union bound

$$
\begin{aligned}
\mathbf{P}\left(\sum_{\mathbb{B} \in \mathcal{B}_{k, \ell}} d_{\mathbb{B}, K^{-\gamma \theta_{0}} \delta, R}(\text { hard }) \geq w\right) & \leq 12 \mathbf{P}\left(d_{\mathbb{B}(S), K^{-\gamma \theta_{0}} \delta, R}(\text { hard }) \geq w / 12\right) \\
& =12 \mathbf{P}\left(d_{\mathbb{B}\left(K^{\eta} S\right), \delta, K^{\eta} R}(\text { hard }) \geq w / 12\right),
\end{aligned}
$$

where the equality is by (2.12) and Proposition 2.35. Let $\mathcal{R}_{L}$ be the set of paths in $\mathcal{G}$ which start at the top of $\mathcal{G}$ and have length $L$, where only nearest-neighbor (horizontal and vertical, not diagonal) edges are allowed. We note that

$$
\left|\mathcal{R}_{L}\right| \leq L 4^{L} \leq 5^{L} .
$$

If there is no $\omega \in Q$ so that $\sum_{\mathbb{B} \in \mathcal{B}_{k, \ell}} d_{\mathbb{B}, K^{-\gamma \theta_{0}} \delta, R}$ (hard) $\leq w$ for all $(k, \ell) \in \omega$, then by planar duality there must be a $\xi \in \mathcal{R}_{L}$ so that $\sum_{\mathbb{B} \in \mathcal{B}_{k, \ell}} d_{\mathbb{B}, K^{-\gamma \theta_{0}} \delta, R}$ (hard) $\geq w$ for all $(k, \ell) \in \xi$. Therefore,

$$
\begin{aligned}
& \mathbf{P}\left(\min _{\omega \in Q} \sum_{j=1}^{J(\omega)} \sum_{\mathbb{B} \in \mathcal{B}_{k, \ell}} d_{\mathbb{B}, K^{-\gamma \theta_{0}}} \delta, R\right. \\
& \quad \leq\left|\mathcal{R}_{L}\right| \max _{\xi \in \mathcal{R}_{L}} \mathbf{P}\left(\bigcap_{(k, \ell) \in \xi}\left\{\sum_{\mathbb{B} \in \mathcal{B}_{k, \ell}} d_{\mathbb{B}, K^{-\gamma} \theta_{0} \delta, R}(\text { hard }) \geq w\right\}\right) \leq \mathbf{P}\left(\bigcup_{\xi \in \mathcal{R}_{L}(k, \ell) \in \xi}\left\{\sum_{\mathbb{B} \in \mathcal{B}_{k, \ell}} d_{\mathbb{B}, K^{-\gamma \theta_{0}} \delta, R}(\text { hard }) \geq w\right\}\right)
\end{aligned}
$$


Now for any $\xi \in \mathcal{R}_{L}$, we have

$$
\begin{aligned}
& \mathbf{P}\left(\bigcap_{(k, \ell) \in \xi}\left\{\sum_{\mathbb{B} \in \mathcal{B}_{k, \ell}} d_{\mathbb{B}, K^{-\gamma \theta_{0}} \delta, R}(\text { hard }) \geq w\right\}\right) \leq \min _{m=1}^{M} \mathbf{P}\left(\bigcap_{(k, \ell) \in \xi \cap \mathcal{G}_{m}}\left\{\sum_{\mathbb{B} \in \mathcal{B}_{k, \ell}} d_{\mathbb{B}, K^{-\gamma \theta_{0}} \delta, R}(\text { hard }) \geq w\right\}\right) \\
& \quad \leq \min _{m=1}^{M} \prod_{(k, \ell) \in \mathcal{G}_{m}} \mathbf{P}\left(\sum_{\mathbb{B} \in \mathcal{B}_{k, \ell}} d_{\mathbb{B}, K^{-\gamma \theta_{0}} \delta, R}(\text { hard }) \geq w\right) \leq \min _{m=1}^{M}\left[12 \mathbf{P}\left(d_{\mathbb{B}\left(K^{\eta} S\right), \delta, K^{\eta} R}(\text { hard }) \geq w / 12\right)\right]^{\left|\xi \cap \mathcal{G}_{m}\right|} \\
& \quad \leq\left[12 \mathbf{P}\left(d_{\mathbb{B}\left(K^{\eta} S\right), \delta, K^{\eta} R}(\text { hard }) \geq w / 12\right)\right]^{L / M},
\end{aligned}
$$

where in the third inequality we used (4.26). The right-hand side does not depend on $\xi$, so we have by (4.27) and (4.28) that

$$
\mathbf{P}\left(\min _{\omega \in Q} \sum_{j=1}^{J(\omega)} \sum_{\mathbb{B} \in \mathcal{B}_{k, \ell}} d_{\mathbb{B}, K^{-\gamma \theta_{0}} \delta, R}(\text { hard }) \geq 12 K L w\right) \leq\left[5\left[12 \mathbf{P}\left(d_{\mathbb{B}\left(K^{\eta} S\right), \delta, K^{\eta} R}(\text { hard }) \geq w / 12\right]^{1 / M}\right]^{L} .\right.
$$

Now we take $w=12 \Theta_{\mathbb{B}(K S), \delta, K R}^{\text {hard }}(1-p)$, with $p<1 /\left(12 \cdot 10^{M}\right)$, so

$$
\mathbf{P}\left(\min _{\omega \in Q} \sum_{j=1}^{J(\omega)} \sum_{\mathbb{B} \in \mathcal{B}_{k, \ell}} d_{\mathbb{B}, K^{-\gamma \theta_{0}} \delta, R}(\text { hard }) \geq 12 K L \Theta_{\mathbb{B}\left(K^{\eta} S\right), \delta, K^{\eta} R}^{\text {hard }}(1-p)\right) \leq\left[5 \cdot(12 p)^{1 / M}\right]^{L} \leq 2^{-L} .
$$

Recalling (4.25), (4.24), and the definition of $L \in\{K / 3,3 K\}$, we obtain, for all $v>1$,

$$
\mathbf{P}\left(d_{\mathbb{R}, \delta, R}(\mathrm{~L}, \mathrm{R}) \geq 36 v K^{2} \Theta_{\mathbb{B}\left(K^{\eta} S\right), \delta, K^{\eta} R}^{\mathrm{hard}}(1-p)\right) \leq C\left(2^{-K / 3}+\mathrm{e}^{-\frac{(\log v)^{2}}{2 \gamma^{2} \log K}}\right) .
$$

Now taking $v=\mathrm{e}^{K^{3 / 4}}$ yields (4.4), while taking $v=C \mathrm{e}^{(\log K)^{3 / 4}}$ for an appropriate constant $C$ and taking $K$ sufficiently large (depending on the choice of $p$ ) yields (4.5).

\section{Fluctuations of the crossing distance: Efron-Stein inequality and multi- scale analysis}

In this section, we prove the following theorem, bounding the variance of the hard crossing distance at a given scale by an absolute constant. Recall Definition 4.3 of $\chi_{U}$.

Theorem 5.1. There is a constant $C<\infty$ so that

$$
\sup _{U \in(0, \infty)} \chi_{U} \leq C
$$

and

$$
\operatorname{Var}\left(\log d_{\mathbb{B}(U)}(\text { hard })\right) \leq C
$$

for all $U \geq 0$.

Before we prove Theorem 5.1, we point out some corollaries.

Corollary 5.2. There is an increasing function $\Theta^{*}: \mathbf{R}_{>0} \rightarrow \mathbf{R}_{>0}$ so that for any $0<p<1$ there is a constant $C=C(p)$ so that, for all $U \in(0, \infty)$,

$$
\Theta_{\mathbb{B}(U)}^{\text {easy }}(p), \Theta_{\mathbb{B}(U)}^{\text {hard }}(p) \in\left[C^{-1} \Theta^{*}(U), C \Theta^{*}(U)\right] .
$$


Proof. Take $\Theta^{*}(U)=\Theta_{\mathbb{B}(U)}^{\text {hard }}(1 / 2)$. The fact that $\Theta^{*}$ is increasing then follows from Proposition 2.36. The fact that, for any $p \in(0,1)$, there is a constant $C=C(p)$ so that, for all $U>0$, we have $\Theta_{\mathbb{B}(U)}^{\text {hard }}(p) \in$ $\left[C^{-1} \Theta^{*}(U), C \Theta^{*}(U)\right]$ is a consequence of Lemma A.1 in light of (5.2). Therefore, by Theorem 3.4 we have, for all $q \in(0,1)$ and $U \in(0, \infty)$, that

$$
\Theta_{\mathbb{B}(U)}^{\text {easy }}(q) \geq \frac{1}{C_{\mathrm{RSW}}} \Theta_{\mathbb{B}(U)}^{\mathrm{hard}}\left(q / C_{\mathrm{RSW}}\right) \geq \frac{1}{C\left(q / C_{\mathrm{RSW}}\right) \cdot C_{\mathrm{RSW}}} \Theta^{*}(U) .
$$

On the other hand, we also have from (4.13) of Proposition 4.6, Proposition 2.36, and Lemma A.1 in light of (5.2) that for every $p \in(0,1)$ there are constants $c>0, C<\infty$ and $K \in[1, \infty)$ so that, for all $U \in(0, \infty)$,

$$
\Theta_{\mathbb{B}(U)}^{\text {easy }}(p) \leq K^{C} \Theta_{\mathbb{B}\left(K^{-1} U\right)}^{\text {hard }}\left(p_{1}\right) \leq K^{C} \Theta_{\mathbb{B}(U)}^{\text {hard }}\left(p_{1}\right) \leq C K^{C} \Theta^{*}(U) .
$$

Thus we have (5.3).

As noted in the introduction, the proof of our main result works without precise knowledge of the growth rate of the quantiles, hence without precise knowledge of $\Theta^{*}(U)$. However, we do know that the growth of $\Theta^{*}(U)$ is bounded above and below by power laws.

Corollary 5.3. We have a constant $C<\infty$ so that, for all $U \geq 1$, we have

$$
\Theta^{*}(U) \in\left[C^{-1} U^{C^{-1}}, C U^{C}\right] .
$$

Proof. This follows from Corollary 5.2, relating $\Theta^{*}(U)$ to the easy and hard crossing quantiles, and Propositions 4.4 and 4.6, which give power-law upper and lower bounds on those quantiles.

We will see that Corollary 5.3 implies Theorem 1.3, as $\Theta^{*}(U)$ will be the normalizing constant for the metric. (See Corollary 6.2 below.)

Our proof of Theorem 5.1 will be by induction on the scale $S$. Throughout the argument in this section, we will fix a scale $S$ and let $\mathbb{R}=\mathbb{B}(K S, L S)$, where $K / L \in[1 / 3,3]$. The scale $S$ will be our inductive parameter and $K$ will represent the increment of scales that we use in our induction. Ultimately, $K$ will be chosen to be very large but fixed.

The inductive procedure relies on the Efron-Stein inequality. In Subsection 5.1, we describe a procedure for breaking up an underlying white noise for the Gaussian free field into independent chunks, each of which can be resampled independently. One of the chunks corresponds to a smooth "coarse" part of the GFF, while the rest of the chunks correspond to the "fine field" on a small part of the domain. We apply the EfronStein inequality and describe the induction procedure in Subsection 5.3. The induction procedure works by assuming that (5.1) holds at scale $S$, and then proving that it still holds at scale $K S$, for the same constant $C$. The analysis required to show that $C$ and $K$ can be chosen so that the induction closes is rather delicate.

To make the induction work, we need to bound the result of the Efron-Stein inequality, which means we need to estimate how much the crossing distance of the box changes when each piece of the white noise is resampled. When we resample the coarse field, this comes from Gaussian concentration as described in Subsection 5.3.1. When we resample the fine field, this involves breaking up the geodesic into a piece which is close to the resampled box and a part that is far away from the resampled box; this construction is described in Subsection 5.2. To estimate the increase in the number of LQG balls needed to cover the "far away" part of the geodesic, we don't need to obtain a new geodesic for the resampled field, since the far away part of the GFF will change by a smooth function. (See Subsection 5.3.2.) On the other hand, to estimate the increase in the number of LQG balls needed to cover the "close" part of the geodesic, we replace the geodesic by an encircling annular crossing optimized for the resampled field. (See Subsection 5.3.3.) Throughout the arguments, the RSW result Theorem 3.4 and the percolation-based bounds of Section 4 will play essential roles in providing the bounds that we need to close the induction. 


\subsection{Resampling the field}

Let $\left(\mathbb{C}_{k}\right)_{k \in Q_{*}}\left(Q_{*}\right.$ being an index set) be a grid partition of $\mathbb{R}^{*}$ into disjoint identical squares of size at most $S$ and at least $S / 10$ so that $\left(\mathbb{C}_{i}\right)_{i \in Q_{\circ}}$ is a partition of $\mathbb{R}^{\circ}$ for some subset $Q_{\circ} \subset Q_{*}$. We note that there is a constant $C$ so that $\left|Q_{*}\right| \leq C K^{2}$.

We note that there is a space-time white noise $W$ on $\mathbb{R}^{*} \times(0, \infty)$ so that we can write

$$
h_{\mathbb{R}^{*}}(x)=\sqrt{\pi} \int_{0}^{\infty} \int_{\mathbb{R}^{*}} p_{t / 2}^{\mathbb{R}^{*}}(x, y) W(\mathrm{~d} y \mathrm{~d} t),
$$

where $p_{t}^{\mathbb{R}^{*}}$ is the transition kernel at time $t$ for a standard Brownian motion killed on the boundary of $\mathbb{R}^{*}$. (This can be verified by checking that this yields the covariance function (2.1).) Note that the symbol $h_{\mathbb{R}^{*}}(x)$ is an abuse of notation since the Gaussian free field does not take pointwise values: (5.4) should be interpreted in the sense of distributions. Now let $\widetilde{W}$ denote a space-time white noise on $(0, \infty) \times \mathbb{R}^{*}$ which is independent of $W$. Define (again in the sense of distributions)

$$
h_{\mathbb{R}^{*}}^{(i)}(x)=\sqrt{\pi} \int_{0}^{S^{2}} \int_{\mathbb{C}_{i}} p_{t / 2}^{\mathbb{R}^{*}}(x, y) \widetilde{W}(\mathrm{~d} y \mathrm{~d} t)+\sqrt{\pi} \iint_{\left(\mathbf{R}_{>0} \times \mathbb{R}^{*}\right) \backslash\left(\left(0, S^{2}\right) \times \mathbb{C}_{i}\right)} p_{t / 2}^{\mathbb{R}^{*}}(x, y) W(\mathrm{~d} y \mathrm{~d} t)
$$

for each $i \in Q_{*}$, and

$$
\tilde{h}_{\mathbb{R}^{*}}(x)=\sqrt{\pi} \int_{S^{2}}^{\infty} \int_{\mathbb{R}^{*}} p_{t / 2}^{\mathbb{R}^{*}}(x, y) \widetilde{W}(\mathrm{~d} y \mathrm{~d} t)+\sqrt{\pi} \int_{0}^{S^{2}} \int_{\mathbb{R}^{*}} p_{t / 2}^{\mathbb{R}^{*}}(x, y) W(\mathrm{~d} y \mathrm{~d} t) .
$$

Of course, $h_{\mathbb{R}^{*}}^{(i)}$, for each $i \in Q_{*}$, and $\tilde{h}_{\mathbb{R}^{*}}$ are all Gaussian free fields, identical in law to $h_{\mathbb{R}^{*}}$. As in Subsection 2.2, for all $\mathbb{B} \subset \mathbb{R}^{*}$, define $h_{\mathbb{R}^{*}: \mathbb{B}}^{(i)}$ (resp. $\tilde{h}_{\mathbb{R}^{*}: \mathbb{B}}$ ) to be the harmonic interpolation of $h_{\mathbb{R}^{*}}^{(i)}$ (resp. $\tilde{h}_{\mathbb{R}^{*}}$ ) onto $\mathbb{B}$, and simply $h_{\mathbb{R}^{*}}^{(i)}$ (resp. $\tilde{h}_{\mathbb{R}^{*}}$ ) outside of $\mathbb{B}$. Also define $h_{\mathbb{B}}^{(i)}=h_{\mathbb{R}^{*}}^{(i)}-h_{\mathbb{R}^{*}: \mathbb{B}}^{(i)}$ and $\tilde{h}_{\mathbb{B}}=\tilde{h}_{\mathbb{R}^{*}}-\tilde{h}_{\mathbb{R}^{*}: \mathbb{B}}$ on $\mathbb{B}$.

For all $\mathbb{B} \subset \mathbb{R}^{*}$, let $\mu_{\mathbb{B}}^{(i)}$ (resp. $\tilde{\mu}_{\mathbb{B}}$ ) denote the Liouville quantum gravity measure on $\mathbb{B}$ using the GFF $h_{\mathbb{B}}^{(i)}$ (resp. $\tilde{h}_{\mathbb{B}}$ ) and let $d_{\mathbb{B}, \ldots}^{(i)}$ (resp. $\tilde{d}_{\mathbb{B}, \ldots}$ ) denote the corresponding Liouville graph distance. Thus $d_{\mathbb{B}, \ldots}^{(i)}$ and $\tilde{d}_{\mathbb{B}, \ldots}$ are identical in law to $d_{\mathbb{B}, \ldots}$. The only difference is that they are defined using different realizations of the underlying white noise field.

\subsection{Splitting the geodesic}

To control the variance of the LFPP crossing distance, we will use the Efron-Stein inequality to control the effect of resampling each piece of the white noise on the crossing distance. The idea is that, resampling the white noise on a small box only has a significant effect on the weight of the part of the geodesic close to that box. Put simply, by breaking up the white noise and also breaking up the geodesic, we can control the influence of each part of the white noise on each part of the geodesic. In the last subsection, we described how we break up the white noise. In this one, we describe how we break up the geodesic.

Suppose that $\pi$ is an $\left(\mathbb{R}^{*}, \mathbb{R}, 1, S\right)$-geodesic of $\mathbb{R}$ and let $B_{1}, \ldots, B_{N}$ be the corresponding sequence of geodesic balls, so $N$ is the LQG distance between the two endpoints of $\pi$. Fix a parameterization of $\pi$, which we will also call $\pi:[0,1] \rightarrow \mathbb{R}$. Define

$$
\mathcal{T}=\pi^{-1}\left(\bigcup_{n=1}^{N-1}\left(B_{n} \cap B_{n+1}\right)\right)
$$

Let $\tau_{0}=0$, and inductively define $\tau_{m}=1 \wedge \min \left\{\tau \geq \tau_{m-1}: \tau \in \mathcal{T}\right.$ and $\left.\operatorname{diam}_{\mathrm{E}}\left(\pi\left(\left[\tau_{m-1}, \tau\right]\right)\right) \geq S\right\}$. Let $M$ be the first $m$ so that $\tau_{m}=1$. For each $m=1, \ldots, M$, let $x_{m}=\pi\left(\tau_{m}\right)$ and let $\pi_{m}=\pi\left(\left[\tau_{m-1}, \tau_{m}\right]\right)$. Thus $\pi$ is the union of $\pi_{1}, \ldots, \pi_{M}$. By the definition of $\mathcal{T}$ and the fact that the $B_{i}$ s have radius at most $S$, we have

$$
\operatorname{diam}_{\mathrm{E}}\left(\pi_{m}\right) \leq 2 S \text {. }
$$


Also, by the definition of $\mathcal{T}$ and the triangle inequality, we have

$$
\sum_{m=1}^{M} d_{\mathbb{R}, 1, S}\left(x_{m-1}, x_{m}\right)=d_{\mathbb{R}, 1, S}(\mathrm{~L}, \mathrm{R}) .
$$

We will need a way to identify which parts of our path are "close" to a given box $\mathbb{C}_{i}$. For $i \in Q_{*}$, define

$$
\mathcal{N}(i)=\left\{j \in Q_{\circ}: \operatorname{dist}_{\mathrm{E}}\left(\mathbb{C}_{i}, \mathbb{C}_{j}\right)<4 S\right\}
$$

to be the set of indices of "nearby" boxes to $\mathbb{C}_{i}$, and define

$$
\mathbb{D}_{i}=\bigcup_{j \in \mathcal{N}(i)} \mathbb{C}_{j}
$$

as the union of these boxes. (Thus, $\mathbb{D}_{i}$ is a larger box surrounding $\mathbb{C}_{i}$.) Also define

$$
\mathcal{D}(i)=Q_{\circ} \backslash \mathcal{N}(i)=\left\{j \in Q_{\circ}: \operatorname{dist}_{\mathrm{E}}\left(\mathbb{C}_{i}, \mathbb{C}_{j}\right) \geq 4 S\right\} .
$$

For $i \in Q_{*}$, we then define

$$
\mathcal{M}(i)=\left\{1 \leq m \leq M: \pi_{m} \cap \mathbb{D}_{i} \neq \emptyset\right\},
$$

the set of segments $\pi_{m}$ that "get close to" $\mathbb{C}_{i}$. Define

$$
\pi_{i}^{-}=\pi \backslash \bigcup_{m \in \mathcal{M}(i)} \pi_{m}
$$

the part of $\pi$ comprised of segments which do not get too close to $\mathbb{C}_{i}$. We note for later use that there is a constant $C<\infty$ so that

$$
\#\left\{j \in Q_{\circ}: \pi_{m} \cap \mathbb{C}_{j} \neq \emptyset\right\} \leq C
$$

and that

$$
\#\left\{i \in Q_{*}: m \in \mathcal{M}(i)\right\} \leq C .
$$

Now define

$$
\mathbb{E}_{i}=\bigcup_{\substack{j \in Q_{\circ} \\ \operatorname{dist}_{\mathrm{E}}\left(\mathbb{C}_{i}, \mathbb{C}_{j}\right)<8 S}} \mathbb{C}_{j},
$$

which is an even larger box around $\mathbb{D}_{i}$; that is, we have $\mathbb{C}_{i} \subset \mathbb{D}_{i} \subset \mathbb{E}_{i}$. Then whenever $m \in \mathcal{M}(i)$, we have $\pi_{m} \subset \mathbb{E}_{i}$ by (5.5). That is, $\mathbb{E}_{i}$ completely contains all segments of the geodesic which get close (in our sense) to $\mathbb{C}_{i}$. By Proposition 2.28, we therefore have

$$
d_{\mathbb{R}, 1, S}\left(x_{m-1}, x_{m}\right)=d_{\mathbb{R}^{*}, \mathbb{E}_{i}, 1, S}\left(x_{m-1}, x_{m}\right) .
$$

Another consequence of the fact $\pi_{m} \subset \mathbb{E}_{i}$ is that $\pi_{m}$ can be "replaced" in the path $\pi$ by a loop around an annulus surrounding $\mathbb{E}_{i}$. That is, if we define

$$
\mathbb{A}_{i}=\left(3 \mathbb{E}_{i} \backslash \mathbb{E}_{i}\right) \cap \mathbb{R}
$$

then we have

$$
d_{\mathbb{R}, 1, S}^{(i)}(\mathrm{L}, \mathrm{R}) \leq d_{\mathbb{R}, 1, S}^{(i)}\left(\pi_{i}^{-}\right)+d_{\mathbb{R}^{*}, \mathbb{A}_{i}, 1, S}^{(i)}(\text { around })
$$

The motivation for using (5.14) will be that, when we resample the white noise in box $\mathbb{C}_{i}$, we will not have pointwise control on the change in the Gaussian free field close to $\mathbb{C}_{i}$. Therefore, there is no reason to believe that the paths $\pi_{m}, m \in \mathcal{M}(i)$, will still be close to geodesics after the resampling. Instead, we will to replace the $\pi_{m}$ s by a geodesic circuit around an annulus, which will be optimized with respect to the resampled field. 


\subsection{The Efron-Stein argument and induction procedure}

We now describe how to apply the Efron-Stein inequality to obtain a bound on the variance. Because our procedure is inductive, we will need to consider separately an error term coming from the event that the coarse field on a given box is larger than $\theta_{0} \log K$, where $\theta_{0}$ is as in (2.12): if this does not hold, then the small boxes may not in fact look smaller than $\mathbb{R}$ when the coarse field is taken into account. We define the following events, recalling the definition (5.8) of $\mathcal{D}(i)$ for each $i \in Q_{*}$ :

$$
\begin{aligned}
\mathcal{A}_{1 ; i} & =\left\{\max _{x \in\left(3 \mathbb{E}_{i}\right)^{\circ}} \max \left\{h_{\mathbb{R}^{*}:\left(3 \mathbb{E}_{i}\right)^{*}}^{(i)}(x), h_{\mathbb{R}^{*}:\left(3 \mathbb{E}_{i}\right)^{*}}(x)\right\} \leq \theta_{0} \log K\right\} ; \\
\mathcal{A}_{2 ; i} & =\left\{\max _{j \in \mathcal{D}(i)} \max _{x \in\left(3 \mathbb{E}_{j}\right)^{\circ}} \max \left\{h_{\mathbb{R}^{*}:\left(3 \mathbb{E}_{j}\right)^{*}}^{(i)}(x), h_{\mathbb{R}^{*}:\left(3 \mathbb{E}_{j}\right)^{*}}(x)\right\} \leq \theta_{0} \log K+S^{-1} \operatorname{dist}_{\mathbb{E}}\left(\mathbb{C}_{i}, \mathbb{C}_{j}^{\circ}\right)\right\} ; \\
\mathcal{A}_{i} & =\mathcal{A}_{1 ; i} \cap \mathcal{A}_{2 ; i} .
\end{aligned}
$$

Lemma 5.4. We have a constant $C$ so that $\mathbf{P}\left(\mathcal{A}_{i}^{c}\right) \leq C K^{-\theta_{0}^{2} / 2}$.

Proof. We note that there is a constant $C$ so that

$$
\mathbf{P}\left(\mathcal{A}_{1 ; i}^{c}\right) \leq C K^{-\theta_{0}^{2} / 2}
$$

by (2.5). Also, we have

$$
\begin{aligned}
& \mathbf{P}\left(\max _{x \in \mathbb{E}_{j}^{\circ}} \max \left\{h_{\mathbb{R}^{*}: \mathbb{E}_{i}^{*}}^{(i)}(x), h_{\mathbb{R}^{*}: \mathbb{E}_{i}^{*}}(x)\right\} \leq \theta_{0} \log K+S^{-1} \operatorname{dist}_{\mathbb{E}}\left(\mathbb{C}_{i}, \mathbb{C}_{j}^{\circ}\right)\right) \\
& \leq 2 \mathbf{P}\left(\max _{x \in \mathbb{E}_{j}^{\circ}} h_{\mathbb{R}^{*}: \mathbb{E}_{i}^{*}}(x) \leq\left(\theta_{0}+\frac{\operatorname{dist}_{\mathrm{E}}\left(\mathbb{C}_{i}, \mathbb{C}_{j}^{\circ}\right)}{S \log K}\right) \log K\right) \\
& \quad \leq C \exp \left\{-\frac{1}{2}\left(\theta_{0}+\frac{\operatorname{dist}\left(\mathbb{C}_{i}, \mathbb{C}_{j}^{\circ}\right)}{S \log K}\right)^{2} \log K\right\} \leq C K^{-\theta_{0}^{2} / 2} \exp \left\{-\frac{\left(\theta_{0}+2\right) \operatorname{dist}\left(\mathbb{C}_{i}, \mathbb{C}_{j}^{\circ}\right)}{2 S}\right\},
\end{aligned}
$$

where the second inequality is by (2.5). Therefore, by a union bound, we obtain

$$
\mathbf{P}\left(\mathcal{A}_{2 ; i}\right) \leq C K^{-\theta_{0}^{2} / 2} \sum_{j \in \mathcal{D}(i)} \exp \left\{-\frac{\left(\theta_{0}+2\right) \operatorname{dist}\left(\mathbb{C}_{i}, \mathbb{C}_{j}^{\circ}\right)}{2 S}\right\} \leq C K^{-\theta_{0}^{2} / 2} .
$$

Taking a union bound over (5.18) and (5.19), we obtain the conclusion of the lemma.

Now we are ready to state the result of our application of the Efron-Stein inequality.

\section{Lemma 5.5. We have}

$\operatorname{Var}\left(\log d_{\mathbb{R}}(\mathrm{L}, \mathrm{R})\right)$

$$
\begin{aligned}
& \leq \mathbf{E}\left[\left(\log \frac{\tilde{d}_{\mathbb{R}, 1, S}(\mathrm{~L}, \mathrm{R})}{d_{\mathbb{R}, 1, S}(\mathrm{~L}, \mathrm{R})}\right)^{2}\right]+2 \sum_{i \in Q_{*}} \mathbf{E}\left[\mathbf{1}_{\mathcal{A}_{i}}\left(\left(\frac{d_{\mathbb{R}, 1, S}^{(i)}\left(\pi_{i}^{-}\right)}{d_{\mathbb{R}, 1, S}(\pi)}-1\right)^{+}\right)^{2}\right]+2 \operatorname{Var}\left(\log \frac{d_{\mathbb{R}, 1, S}(\mathrm{~L}, \mathrm{R})}{d_{\mathbb{R}}(\mathrm{L}, \mathrm{R})}\right) \\
& +2\left(\mathbf{E}\left[\max _{i \in Q_{*}} d_{\left(3 \mathbb{E}_{i}\right)^{*}, \mathbb{A}_{i}, K^{-\gamma \theta_{0}, S}}^{(i)}(\operatorname{around})^{3}\right] \mathbf{E}\left[d_{\mathbb{R}, 1, S}(\mathrm{~L}, \mathrm{R})^{-3}\right] \mathbf{E}\left[\left(\sum_{i \in Q_{*}} \frac{\mathbf{1}_{\mathcal{A}_{i}} d_{\mathbb{R}^{*}, \mathbb{A}_{i}, 1, S}^{(i)}(\text { around })}{d_{\mathbb{R}, 1, S}(\mathrm{~L}, \mathrm{R})}\right)^{3}\right]\right)^{1 / 3} \\
& +\sum_{i \in Q_{*}} \mathbf{E}\left[\left(\left(\log \frac{d_{\mathbb{R}, 1, S}^{(i)}(\mathrm{L}, \mathrm{R})}{d_{\mathbb{R}, 1, S}(\mathrm{~L}, \mathrm{R})}\right)^{+}\right)^{2} \mathbf{1}_{\mathcal{A}_{i}^{c}}\right] .
\end{aligned}
$$


Remark 5.6. Before deriving (5.20), we give a brief description of its terms. By symmetry, we only need to consider the potential increase in the distance after resampling the field. To this end, we consider a geodesic path with respect to the original field, and for each type of resampling use it to construct a slightly perturbed path that has a not-too-much-larger LQG graph length with respect to the resampled field. The first term in (5.20) represents the effect of resampling the white noise at times far in the future, which corresponds to resampling a smooth "coarse field." This has a "Lipschitz" effect on the weight of the path, so we can bound it using Gaussian concentration inequalities. The second term represents the effect of resampling the white noise at times close to 0 on the path far away from the resampled region. Here again, the resampling should be smooth, but since we are only considering the effect on part of the path, we need to use a more customized argument. The fourth term represents the effect of resampling the white noise on the path close to the resampled region. In this case, the LQG in the relevant region should change substantially, so we replace the path in this region by an optimal circuit of an annulus surrounding the region. The third term accounts for the error incurred in passing from $d_{\mathbb{R}, 1, S}(\mathrm{~L}, \mathrm{R})$ to $d_{\mathbb{R}}(\mathrm{L}, \mathrm{R})$, which is to say the error incurred by requiring the geodesic balls to have radius at most $S$. This error should be negligible if the scale is large enough; we deal with small scales by the a priori bound (4.7), which was proved in [12]. Finally, the last term is an error term incurred from the possibility that the coarse field could be extraordinarily large, which happens with low probability and thus is bounded in Subsection 5.3.6.

Proof of Lemma 5.5. By the Cauchy-Schwarz inequality, we have

$$
\operatorname{Var}\left(\log d_{\mathbb{R}}(\mathrm{L}, \mathrm{R})\right) \leq 2 \operatorname{Var}\left(\log d_{\mathbb{R}, 1, S}(\mathrm{~L}, \mathrm{R})\right)+2 \operatorname{Var}\left(\log \frac{d_{\mathbb{R}, 1, S}(\mathrm{~L}, \mathrm{R})}{d_{\mathbb{R}}(\mathrm{L}, \mathrm{R})}\right) .
$$

By the Efron-Stein inequality and the decomposition of the white noise described in Subsection 5.1, we have

$$
\operatorname{Var}\left(\log d_{\mathbb{R}}(\mathrm{L}, \mathrm{R})\right) \leq \frac{1}{2} \mathbf{E}\left[\left(\log \frac{\tilde{d}_{\mathbb{R}, 1, S}(\mathrm{~L}, \mathrm{R})}{d_{\mathbb{R}, 1, S}(\mathrm{~L}, \mathrm{R})}\right)^{2}\right]+\frac{1}{2} \sum_{i \in Q_{*}} \mathbf{E}\left[\left(\log \frac{d_{\mathbb{R}, 1, S}^{(i)}(\mathrm{L}, \mathrm{R})}{d_{\mathbb{R}, 1, S}(\mathrm{~L}, \mathrm{R})}\right)^{2}\right] .
$$

We note that the first term of (5.22) corresponds to the resampling of the coarse field, while the second corresponds to the resampling of the fine field. For the present, we satisfy ourselves with further developing the fine field term. Using the exchangeability of the resampled and unresampled white noises, we obtain

$$
\mathbf{E}\left[\left(\log \frac{d_{\mathbb{R}, 1, S}^{(i)}(\mathrm{L}, \mathrm{R})}{d_{\mathbb{R}, 1, S}(\mathrm{~L}, \mathrm{R})}\right)^{2}\right]=2 \mathbf{E}\left[\left(\left(\log \frac{d_{\mathbb{R}, 1, S}^{(i)}(\mathrm{L}, \mathrm{R})}{d_{\mathbb{R}, 1, S}(\mathrm{~L}, \mathrm{R})}\right)^{+}\right)^{2}\right] .
$$

Now we split the right-hand side of (5.23) as

$$
\mathbf{E}\left[\left(\left(\log \frac{d_{\mathbb{R}, 1, S}^{(i)}(\mathrm{L}, \mathrm{R})}{d_{\mathbb{R}, 1, S}(\mathrm{~L}, \mathrm{R})}\right)^{+}\right)^{2}\right]=\mathbf{E}\left[\left(\left(\log \frac{d_{\mathbb{R}, 1, S}^{(i)}(\mathrm{L}, \mathrm{R})}{d_{\mathbb{R}, 1, S}(\mathrm{~L}, \mathrm{R})}\right)^{+}\right)^{2}\left(\mathbf{1}_{\mathcal{A}_{i}}+\mathbf{1}_{\mathcal{A}_{i}^{c}}\right)\right] .
$$

Recalling the elementary inequality $\left(\log \frac{X}{Y}\right)^{+} \leq(X-Y)^{+} / Y$ for $X, Y>0$, we have

$$
\left(\left(\log \frac{d_{\mathbb{R}, 1, S}^{(i)}(\mathrm{L}, \mathrm{R})}{d_{\mathbb{R}, 1, S}(\mathrm{~L}, \mathrm{R})}\right)^{+}\right)^{2} \leq\left(\frac{\left(d_{\mathbb{R}, 1, S}^{(i)}(\mathrm{L}, \mathrm{R})-d_{\mathbb{R}, 1, S}(\mathrm{~L}, \mathrm{R})\right)^{+}}{d_{\mathbb{R}, 1, S}(\mathrm{~L}, \mathrm{R})}\right)^{2}
$$


Now we have by (5.14) that

$$
\begin{aligned}
\left(d_{\mathbb{R}, 1, S}^{(i)}(\mathrm{L}, \mathrm{R})-d_{\mathbb{R}, 1, S}(\mathrm{~L}, \mathrm{R})\right)^{+} & \leq\left(d_{\mathbb{R}, 1, S}^{(i)}\left(\pi_{i}^{-}\right)+d_{\mathbb{R}^{*}, \mathbb{A}_{i}, 1, S}^{(i)}(\text { around })-d_{\mathbb{R}, 1, S}(\pi)\right)^{+} \\
& \leq\left(d_{\mathbb{R}, 1, S}^{(i)}\left(\pi_{i}^{-}\right)-d_{\mathbb{R}, 1, S}(\pi)\right)^{+}+d_{\mathbb{R}^{*}, \mathbb{A}_{i}, 1, S}^{(i)}(\text { around }) .
\end{aligned}
$$

Substituting (5.26) into (5.25), and using the fact that $\pi$ is an $\left(\mathbb{R}^{*}, \mathbb{R}, 1, S\right)$-geodesic, gives us

$$
\begin{aligned}
\left(\left(\log \frac{d_{\mathbb{R}, 1, S}^{(i)}(\mathrm{L}, \mathrm{R})}{d_{\mathbb{R}, 1, S}(\mathrm{~L}, \mathrm{R})}\right)^{+}\right)^{2} & \leq\left(\frac{\left(d_{\mathbb{R}, 1, S}^{(i)}\left(\pi_{i}^{-}\right)-d_{\mathbb{R}, 1, S}(\pi)\right)^{+}+d_{\mathbb{R}^{*}, \mathbb{A}_{i}, 1, S}^{(i)}(\text { around })}{d_{\mathbb{R}, 1, S}(\mathrm{~L}, \mathrm{R})}\right)^{2} \\
& \leq 2\left(\left(\frac{d_{\mathbb{R}, 1, S}^{(i)}\left(\pi_{i}^{-}\right)}{d_{\mathbb{R}, 1, S}(\pi)}-1\right)^{+}\right)^{2}+2\left(\frac{d_{\mathbb{R}^{*}, \mathbb{A}_{i}, 1, S}^{(i)}(\text { around })}{d_{\mathbb{R}, 1, S}(\mathrm{~L}, \mathrm{R})}\right)^{2}
\end{aligned}
$$

Summing the $\mathbf{1}_{\mathcal{A}_{i}}$ part of (5.24), and then applying (5.27), we get

$$
\begin{array}{r}
\sum_{i \in Q_{*}} \mathbf{E}\left[\mathbf{1}_{\mathcal{A}_{i}}\left(\left(\log \frac{d_{\mathbb{R}, 1, S}^{(i)}(\mathrm{L}, \mathrm{R})}{d_{\mathbb{R}, 1, S}(\mathrm{~L}, \mathrm{R})}\right)^{+}\right)^{2}\right] \leq 2 \sum_{i \in Q_{*}} \mathbf{E}\left[\mathbf{1}_{\mathcal{A}_{i}}\left(\left(\frac{d_{\mathbb{R}, 1, S}^{(i)}\left(\pi_{i}^{-}\right)}{d_{\mathbb{R}, 1, S}(\pi)}-1\right)^{+}\right)^{2}\right] \\
+2 \sum_{i \in Q_{*}} \mathbf{E}\left[\mathbf{1}_{\mathcal{A}_{i}}\left(\frac{d_{\mathbb{R}^{*}, \mathbb{A}_{i}, 1, S}^{(i)}(\text { around })}{d_{\mathbb{R}, 1, S}(\mathrm{~L}, \mathrm{R})}\right)\right.
\end{array}
$$

We bound the second term of (5.28) by

$$
\begin{aligned}
& \sum_{i \in Q_{*}} \mathbf{E}\left[\mathbf{1}_{\mathcal{A}_{i}}\left(\frac{d_{\mathbb{R}^{*}, \mathbb{A}_{i}, 1, S}^{(i)}(\text { around })}{d_{\mathbb{R}, 1, S}(\mathrm{~L}, \mathrm{R})}\right)^{2}\right] \leq \mathbf{E}\left[\left(\frac{\max _{i \in Q_{*}} \mathbf{1}_{\mathcal{A}_{i}} d_{\mathbb{R}^{*}, \mathbb{A}_{i}, 1, S}^{(i)}}{d_{\mathbb{R}, 1, S}(\mathrm{~L}, \mathrm{R})}\right)\left(\frac{\left.\sum_{i \in Q_{*}} \mathbf{1}_{\mathcal{A}_{i}} d_{\mathbb{R}^{*}, \mathbb{A}_{i}, 1, S}^{(i)} \text { (around }\right)}{d_{\mathbb{R}, 1, S}(\mathrm{~L}, \mathrm{R})}\right)\right] \\
& \leq\left(\mathbf{E}\left[\max _{i \in Q_{*}} d_{\mathbb{R}^{*}, \mathbb{A}_{i}, 1, S}^{(\text {around })^{3}} \mathbf{1}_{\mathcal{A}_{i}}\right] \mathbf{E}\left[d_{\mathbb{R}, 1, S}(\mathrm{~L}, \mathrm{R})^{-3}\right] \mathbf{E}\left[\left(\sum_{i \in Q_{*}} \frac{\mathbf{1}_{\mathcal{A}_{i}} d_{\mathbb{R}^{*}, \mathbb{A}_{i}, 1, S}^{(\text {around })}}{d_{\mathbb{R}, 1, S}(\mathrm{~L}, \mathrm{R})}\right)^{3}\right]\right)^{1 / 3}
\end{aligned}
$$

We note that by (2.41) and (5.15), we have

$$
d_{\mathbb{R}^{*}, \mathbb{A}_{i}, 1, S}^{(i)} \text { (around) } \mathbf{1}_{\mathcal{A}_{i}} \leq d_{\left(3 \mathbb{E}_{i}\right)^{*}, \mathbb{A}_{i}, K^{-\gamma \theta_{0}, S}}^{(i)} \text { (around); }
$$

applying this in (5.29) and substituting into (5.28), we bound the first term of the sum of (5.24) by

$$
\begin{aligned}
& \sum_{i \in Q_{*}} \mathbf{E}\left[\mathbf{1}_{\mathcal{A}_{i}}\left(\left(\log \frac{d_{\mathbb{R}, 1, S}^{(i)}(\mathrm{L}, \mathrm{R})}{d_{\mathbb{R}, 1, S}(\mathrm{~L}, \mathrm{R})}\right)^{+}\right)^{2}\right] \leq 2 \sum_{i \in Q_{*}} \mathbf{E}\left[\mathbf{1}_{\mathcal{A}_{i}}\left(\left(\frac{d_{\mathbb{R}, 1, S}^{(i)}\left(\pi_{i}^{-}\right)}{d_{\mathbb{R}, 1, S}(\pi)}-1\right)^{+}\right)^{2}\right]+ \\
& +2\left(\mathbf { E } [ \operatorname { m a x } _ { i \in Q _ { * } } d _ { ( 3 \mathbb { E } _ { i } ) ^ { * } , \mathbb { A } _ { i } , K ^ { - \gamma \theta _ { 0 } , S } } ^ { ( i ) } ( \text { around } ) ^ { 3 } ] \mathbf { E } [ d _ { \mathbb { R } , 1 , S } ( \mathrm { L } , \mathrm { R } ) ^ { - 3 } ] \mathbf { E } \left[\left(\sum_{i \in Q_{*}} \frac{\left.\left.\left.\mathbf{1}_{\mathcal{A}_{i}} d_{\mathbb{R}^{*}, \mathbb{A}_{i}, 1, S}^{(\text {around })}\right)^{3}\right]\right)^{1 / 3}}{d_{\mathbb{R}, 1, S}(\mathrm{~L}, \mathrm{R})} .\right.\right.\right.
\end{aligned}
$$

Then (5.20) follows from (5.21), (5.22), (5.23), (5.24) and (5.29). 
Most of the remainder of this section will be devoted to bounding the terms of (5.20). Before we get to that, though, we state the overall bound we obtain, and how we use it to prove Theorem 5.1.

Lemma 5.7. We have constants $0<c, C<\infty$ and $K_{0} \geq 1$ so that, if $K \geq K_{0}$ and $S \geq K^{-\eta} T_{0}$ (where $\eta$ is defined in (2.12) and $T_{0}$ is defined as in Proposition 4.4),

$$
\operatorname{Var}\left(\log d_{\mathbb{R}}(\mathrm{L}, \mathrm{R})\right) \leq C \log K+C K^{-c / 2} \bar{\chi}_{K S / 100}^{3}+C K^{C} S^{-c} .
$$

We will prove Lemma 5.7 in Subsection 5.3.7. First we show how to use Lemma 5.7 to carry out the inductive argument to prove Theorem 5.1.

Proof of Theorem 5.1. We have constants $0<z, Z<\infty$ such that the following holds. First, by Lemma 5.7, we have that whenever $K \geq Z$ and $S \geq K^{-\eta} T_{0}$,

$$
\operatorname{Var}\left(\log d_{\mathbb{R}}(\mathrm{L}, \mathrm{R})\right) \leq Z\left(\log K+K^{-z / 2} \bar{\chi}_{K S / 100}^{3}+K^{Z} S^{-z}\right) .
$$

Moreover, by (4.7), for every $S_{0}>0$, we have

$$
\bar{\chi}_{S_{0}} \leq Z \mathrm{e}^{\left(\log S_{0}\right)^{0.95}}
$$

Finally, by (4.6), for all $U>0$ we have

$$
\chi_{U} \leq Z \exp \left\{Z \sqrt{\operatorname{Var}\left(\log d_{\mathbb{B}(U)}(\text { hard })\right)}\right\} .
$$

At this point we treat the values of $z$ and $Z$ as fixed, and do not let them change throughout the proof as we have been doing for constants labeled $c$ and $C$. We want to choose $K$ and $S_{0}$ so that

$$
\begin{aligned}
K & \geq Z, \\
K^{\eta} S_{0} & \geq T_{0}, \\
K^{Z} S_{0}^{-z} & \leq \log K, \\
Z K^{-z} \mathrm{e}^{3\left(\log S_{0}\right)^{0.95} \vee 3 Z \sqrt{3 Z \log K}} & \leq \log K .
\end{aligned}
$$

We can do this as follows. First, we fix

$$
S_{0}=K^{Z / z+1}
$$

which guarantees that (5.37) holds as long as $K$ is sufficiently large. Then $K^{\eta-1} S_{0}=K^{Z / z+\eta}$, so as long as $K$ is sufficiently large, (5.36) will hold. Finally, for (5.38) to hold, we need

$$
\mathrm{e}^{\left(3(Z / z+z)^{0.95}(\log K)^{0.95}\right) \vee 3 Z \sqrt{3 Z \log K}-z \log K} \leq \log K,
$$

which can be achieved by taking $K$ sufficiently large. Finally, (5.35) of course holds for sufficiently large $K$. Therefore, we can achieve (5.35)-(5.38) by fixing $K$ sufficiently large and then imposing (5.39). Henceforth we assume that $K$ and $S_{0}$ have been chosen in this way.

We note that (5.33) and (5.38) imply that if we put

$$
\chi:=\bar{\chi}_{S_{0}} \vee Z \mathrm{e}^{Z \sqrt{3 Z \log K}}
$$

then

$$
K^{-z} \chi \leq \log K \text {. }
$$


Now note that $\left[0, S_{0}\right] \subset \mathcal{S}_{\chi}$ by (5.40) and the definition of $\mathcal{S}_{\mathcal{X}}$ in Definition 4.3. Suppose that $S$ is such that $K S \geq S_{0}$ and $[0, K S / 100] \subset \mathcal{S}_{\chi}$. By (5.36), we have that

$$
K^{\eta} S=K^{\eta-1} K S \geq K^{\eta-1} S_{0} \geq T_{0},
$$

so by plugging in (5.37) and (5.41) into (5.32), we obtain

$$
\operatorname{Var}\left(\log d_{\mathbb{R}}(\mathrm{L}, \mathrm{R})\right) \leq Z\left(\log K+K^{-z} \chi^{3}+K^{Z} S^{-z}\right) \leq Z\left(\log K+K^{-z} \chi^{3}+K^{Z} S^{-z}\right) \leq 3 Z \log K .
$$

Therefore, by (5.34), we have

$$
\chi_{K S} \leq Z \mathrm{e}^{Z \sqrt{3 Z \log K}} \leq \chi,
$$

so $K S \in \mathcal{S}_{\chi}$ as well. By induction, this implies that $\mathcal{S}_{\chi}=[0, \infty)$. From this, (5.32) implies that the variance $\operatorname{Var}\left(\log d_{\mathbb{R}}(\mathrm{L}, \mathrm{R})\right)$ is bounded uniformly in $S$, hence the result.

Thus it remains to prove Lemma 5.7 by bounding the terms of (5.20). That will be our goal in the following subsections.

\subsubsection{The coarse field effect}

First we address the effect of the coarse field (the first term of (5.20)) using Gaussian concentration.

Lemma 5.8. We have a constant $C$ so that

$$
\mathbf{E}\left[\left(\log \frac{\tilde{d}_{\mathbb{R}, 1, S}(\mathrm{~L}, \mathrm{R})}{d_{\mathbb{R}, 1, S}(\mathrm{~L}, \mathrm{R})}\right)^{2}\right] \leq C \log K .
$$

Proof. Let $\mathcal{F}_{S^{2}}$ denote the $\sigma$-algebra generated by the white noise on $\mathbb{R}^{*} \times\left[0, S^{2}\right]$. We note that

$$
\frac{1}{2} \mathbf{E}\left[\left(\log \frac{\tilde{d}_{\mathbb{R}, 1, S}(\mathrm{~L}, \mathrm{R})}{d_{\mathbb{R}, 1, S}(\mathrm{~L}, \mathrm{R})}\right)^{2}\right]=\mathbf{E} \operatorname{Var}\left(\log d_{\mathbb{R}, 1, S}(\mathrm{~L}, \mathrm{R}) \mid \mathcal{F}_{S^{2}}\right)
$$

Let

$$
h_{\text {coarse }}(x)=\sqrt{\pi} \int_{S^{2}}^{\infty} \int_{\mathbb{R}^{*}} p_{t / 2}^{\mathbb{R}^{*}}(x, y) W(\mathrm{~d} y \mathrm{~d} t) .
$$

Now we claim that there is a constant $C<\infty$ so that, for all $x \in \mathbb{R}^{\circ}$, we have

$$
\sup _{x \in \mathbb{R}^{\circ}} \operatorname{Var}\left(h_{\text {coarse }}(x)\right) \leq C \log K \text {. }
$$

This is because we can write

$$
h_{\text {coarse }}(x)=\int_{\mathbb{R}^{*}} p_{S^{2} / 2}^{\mathbb{R}^{*}}(x, y) h_{\mathbb{R}^{*}}(y) \mathrm{d} y
$$

from this is it is clear that the pointwise variance of $h_{\text {coarse }}$ is finite, and (5.44) comes from rescaling so that $S=1$ and then using (2.3).

Since $\log d_{\mathbb{R}, 1, S}$ is measurable with respect to the $\sigma$-algebra generated by $h_{\text {fine }}$ and $h_{\text {coarse }}$, there is a deterministic functional $\widetilde{\mathscr{D}}$ so that

$$
\log d_{\mathbb{R}, 1, S}(\mathrm{~L}, \mathrm{R})=\widetilde{\mathscr{D}}\left(\left.W\right|_{\mathbb{R}^{*} \times\left[0, S^{2}\right]}, h_{\text {coarse }}\right)
$$

with probability 1 . Let $\mathscr{D}$ be an $\mathcal{F}_{S^{2}}$-measurable random functional on $L^{\infty}\left(\mathbb{R}^{\circ}\right)$ given by

$$
\mathscr{D}(h)=\widetilde{\mathscr{D}}\left(\left.W\right|_{\mathbb{R}^{*} \times\left[0, S^{2}\right]}, h\right) .
$$


We note that, with probability $1, \mathscr{D}$ is a $\gamma$-Lipschitz functional on $L^{\infty}\left(\mathbb{R}^{\circ}\right)$. This is to say that we have, for all $\Delta \in L^{\infty}\left(\mathbb{R}^{\circ}\right)$, that

$$
\left|\mathscr{D}\left(h_{\text {coarse }}+\Delta\right)-\mathscr{D}\left(h_{\text {coarse }}\right)\right| \leq \gamma\|\Delta\|_{L^{\infty}\left(\mathbb{R}^{\circ}\right)}
$$

by (2.25) and (2.29).

Let $Q$ be the median value of $\mathscr{D}\left(h_{\text {coarse }}\right)$, conditional on $\mathcal{F}_{S^{2}}$, so $Q$ is an $\mathcal{F}_{S^{2}}$-measurable random variable so that

$$
\mathbf{P}\left(\mathscr{D}\left(h_{\text {coarse }}\right) \leq Q \mid \mathcal{F}_{S^{2}}\right), \mathbf{P}\left(\mathscr{D}\left(h_{\text {coarse }}\right) \geq Q \mid \mathcal{F}_{S^{2}}\right) \geq \frac{1}{2}
$$

almost surely. Let $\mathcal{X}=\left\{h \in L^{\infty}\left(\mathbb{R}^{\circ}\right): \mathscr{D}\left(h_{\text {coarse }}\right) \leq Q\right\}$, so $\mathbf{P}\left(\mathscr{D}\left(h_{\text {coarse }}\right) \in \mathcal{X} \mid \mathcal{F}_{S^{2}}\right) \geq \frac{1}{2}$ almost surely. We can then apply (an infinite-dimensional version of) the Gaussian concentration inequality given in [12, Lemma 2.1], along with (5.44), to observe that

$$
\mathbf{P}\left(\log d_{\mathbb{R}, 1, S}(\mathrm{~L}, \mathrm{R}) \geq Q+\lambda \mid \mathcal{F}_{S^{2}}\right) \leq \mathbf{P}\left(\min _{h \in \mathcal{X}}\left\|h_{\text {coarse }}-h\right\|_{L^{\infty}\left(\mathbb{R}^{\circ}\right)} \geq \gamma^{-1} \lambda \mid \mathcal{F}_{S^{2}}\right) \leq C \exp \left\{-\frac{(\lambda-C \sqrt{\log K})^{2}}{C \log K}\right\}
$$

almost surely. A similar argument implies that

$$
\mathbf{P}\left(\log d_{\mathbb{R}, 1, S}(\mathrm{~L}, \mathrm{R}) \leq X-\lambda \mid \mathcal{F}_{S^{2}}\right) \leq C \exp \left\{-\frac{(\lambda-C \sqrt{\log K})^{2}}{C \log K}\right\}
$$

almost surely. This implies that $\operatorname{Var}\left(\log d_{\mathbb{R}, 1, S}(\mathrm{~L}, \mathrm{R}) \mid \mathcal{F}_{S^{2}}\right) \leq C \log K$ almost surely, which means that

$$
\mathbf{E} \operatorname{Var}\left(\log d_{\mathbb{R}, 1, S}(\mathrm{~L}, \mathrm{R}) \mid \mathcal{F}_{S^{2}}\right) \leq C \log K
$$

implying (5.42) by (5.43).

\subsubsection{The far fine field effect}

Now we turn to the second term of (5.20). First we deal with the part of the path which is "far away" from the white noise being resampled. In this case we don't need to change the path when we resample to get our bound. Rather, we simply bound the increase in the weight of the path by the maximum of the increase in the LQG measure. The bound we obtain in Lemma 5.10 is in terms of the maximum annular circuit distance and the total crossing distance. These terms will also appear in the close fine field bound in the next section, so we wait to bound them together in Subsection 5.3.4.

Lemma 5.9. For every $\lambda>0$, there is a $C<\infty$ so that, for all $i \in Q_{*}$ and $j \in \mathcal{D}(i)$, we have

$$
\mathbf{E} \exp \left\{\lambda\left\|h_{\mathbb{R}^{*}}-h_{\mathbb{R}^{*}}^{(i)}\right\|_{L^{\infty}\left(\mathbb{C}_{j}^{\circ}\right)}\right\} \leq C .
$$

Moreover, if we define

$$
G^{*}=\max _{\substack{i \in Q_{*} \\ j \in \mathcal{D}(i)}}\left(\left\|h_{\mathbb{R}^{*}}-h_{\mathbb{R}^{*}}^{(i)}\right\|_{L^{\infty}\left(\mathbb{C}_{j}^{\circ}\right)} \exp \left\{\frac{\operatorname{dist}_{\mathrm{E}}\left(\mathbb{C}_{i}, \mathbb{C}_{j}^{\circ}\right)^{2}}{C S^{2}}\right\}\right)
$$

(where $\mathcal{D}(i)$ is defined as in (5.8)) then we have

$$
\mathbf{E} \exp \left\{\lambda G^{*}\right\} \leq C \mathrm{e}^{C \sqrt{\log K}} .
$$


Proof. Let $j \in \mathcal{D}(i)$. Define $D=\operatorname{dist}_{\mathrm{E}}\left(\mathbb{C}_{i}, \mathbb{C}_{j}^{\circ}\right)$ and note that $D>0$ by (5.8). We have, a constant $C$, independent of $i, j, D$, so that for $x \in \mathbb{C}_{j}^{\circ}$,

$$
\begin{aligned}
& \operatorname{Var}\left(h_{\mathbb{R}^{*}}^{(i)}(x)-h_{\mathbb{R}^{*}}(x)\right)=2 \pi \int_{0}^{S^{2}} \int_{\mathbb{C}_{i}}\left|p_{t / 2}^{\mathbb{R}^{*}}(x, y)\right|^{2} \mathrm{~d} y \mathrm{~d} t \leq C \int_{0}^{S^{2}} \int_{\mathbb{C}^{2}} t^{-2} \exp \left\{-\frac{|x-y|^{2}}{C t}\right\} \mathrm{d} y \mathrm{~d} t \\
& \quad \leq C \int_{\mathbb{C}_{i}} \int_{0}^{S^{2}|x-y|^{-2}}|x-y|^{-2} t^{-2} \mathrm{e}^{-1 /(C t)} \mathrm{d} t \mathrm{~d} y \leq C D^{-2} S^{2} \int_{0}^{S^{2} D^{-2}} t^{-2} \mathrm{e}^{-1 /(C t)} \mathrm{d} t \leq C \mathrm{e}^{-(D / S)^{2} / C} .
\end{aligned}
$$

Also, for $x, z \in \mathbb{C}_{j}^{\circ}$, using Lemma A.3, we see that

$$
\begin{aligned}
& \operatorname{Var}\left(\left(h_{\mathbb{R}^{*}}^{(i)}(x)-h_{\mathbb{R}^{*}}(x)\right)-\left(h_{\mathbb{R}^{*}}^{(i)}(z)-h_{\mathbb{R}^{*}}(z)\right)\right)=2 \pi \int_{0}^{S^{2}} \int_{\mathbb{C}_{i}}\left|p_{t / 2}^{\mathbb{R}^{*}}(x, y)-p_{t / 2}^{\mathbb{R}^{*}}(z, y)\right|^{2} \mathrm{~d} y \mathrm{~d} t \\
& \quad \leq 2 \pi \int_{0}^{S^{2}} \int_{\mathbb{C}_{i}}|x-z|^{2}\left\|\nabla p_{t / 2}^{\mathbb{R}^{*}}(\cdot, y)\right\|_{L^{\infty}\left(\mathbb{C}_{j}^{\circ}\right)}^{2} \mathrm{~d} y \mathrm{~d} t \leq C|x-z|^{2} S^{2} \int_{0}^{S^{2}} \frac{D^{2}}{t^{4}} \exp \left\{-\frac{2 D^{2}}{t}\right\} \mathrm{d} t \\
& \quad=C|x-z|^{2} S^{2} \int_{0}^{S^{2}} \frac{D^{2}}{t^{4} D^{8}} \exp \left\{-\frac{2 D^{2}}{t D^{2}}\right\} \mathrm{d}\left(D^{2} t\right)=C|x-z|^{2} \frac{S^{2}}{D^{4}} \int_{0}^{S^{2} D^{-2}} \mathrm{e}^{-2 / t} \mathrm{~d} t \\
& \quad=C\left|\frac{x-z}{S}\right|^{2} \frac{S^{4}}{D^{4}} \mathrm{e}^{-(D / S)^{2} / C} .
\end{aligned}
$$

Fernique's inequality ([18]; see e.g. [1, Theorem 4.1]) in light of (5.49) then implies that

$$
\mathbf{E} \sup _{x \in \mathbb{C}_{j}^{\circ}}\left(h_{\mathbb{R}^{*}}^{(i)}(x)-h_{R}(x)\right) \leq C \mathrm{e}^{-(D / S)^{2} / C}=C \exp \left\{-\frac{\operatorname{dist}_{\mathrm{E}}\left(\mathbb{C}_{i}, \mathbb{C}_{j}^{\circ}\right)^{2}}{C S^{2}}\right\} .
$$

Thus, by the Borell-TIS inequality (see e.g. [24, Theorem 7.1], [7, Theorem 6.1], or [1, Theorem 2.1]) and (5.48), we have, for all $u \geq 0$, that

$$
\mathbf{P}\left(\sup _{x \in \mathbb{C}_{j}^{\circ}}\left(h_{\mathbb{R}^{*}}^{(i)}(x)-h_{R}(x)\right) \exp \left\{\frac{\operatorname{dist}_{\mathrm{E}}\left(\mathbb{C}_{i}, \mathbb{C}_{j}^{\circ}\right)^{2}}{C S^{2}}\right\} \geq C+u\right) \leq \mathrm{e}^{-u^{2}} .
$$

Applying the same argument to $-\left(h_{\mathbb{R}^{*}}^{(i)}(x)-h_{R}(x)\right)$ and taking a union bound yields

$$
\mathbf{P}\left(\left\|h_{\mathbb{R}^{*}}^{(i)}-h_{R}\right\|_{L^{\infty}\left(\mathbb{C}_{j}^{\circ}\right)} \exp \left\{\frac{\operatorname{dist}_{\mathrm{E}}\left(\mathbb{C}_{i}, \mathbb{C}_{j}^{\circ}\right)^{2}}{C S^{2}}\right\} \geq C+u\right) \leq \mathrm{e}^{-u^{2}}
$$

Dropping the $\exp \left\{\frac{\operatorname{dist}_{E}\left(\mathbb{C}_{i}, \mathbb{C}_{j}^{\circ}\right)^{2}}{C S^{2}}\right\}$ in (5.50) yields (5.45). On the other hand, taking a union bound over all $i \in Q_{*}, j \in \mathcal{D}(i)$, and noting that the such of all such $i, j$ has size of order $K^{4}$, we obtain (5.47).

Lemma 5.10. There is a constant $C$ so that

$$
\mathbf{E} \sum_{i \in Q_{*}}\left(\left(\frac{d_{\mathbb{R}, 1, S}^{(i)}\left(\pi_{i}^{-}\right)}{d_{\mathbb{R}, 1, S}(\pi)}-1\right)^{+}\right)^{2} \mathbf{1}_{\mathcal{A}_{i}} \leq C \mathrm{e}^{C \sqrt{\log K}}\left(\mathbf{E} \max _{j \in Q_{0}} d_{\left(3 \mathbb{E}_{i}\right)^{*}, \mathbb{A}_{j}, K^{-\gamma \theta_{0, S}}}(\text { around })^{3}\right)^{1 / 3}\left(\mathbf{E} d_{\mathbb{R}, 1, S}(\pi)^{-3}\right)^{1 / 3}
$$


Proof. Note that by (5.9), (2.30), and (2.42) of Proposition 2.38, we have

$$
d_{\mathbb{R}, 1, S}^{(i)}\left(\pi_{i}^{-}\right) \mathbf{1}_{\mathcal{A}_{i}} \leq \sum_{j \in \mathcal{D}(i)} d_{\mathbb{R}, 1, S}^{(i)}\left(\pi_{i}^{-} \cap \mathbb{C}_{j}\right) \mathbf{1}_{\mathcal{A}_{2 ; i}} \leq \mathbf{1}_{\mathcal{A}_{2 ; i}} \sum_{j \in \mathcal{D}(i)} \exp \left\{\gamma\left\|h_{\mathbb{R}^{*}}^{(i)}-h_{\mathbb{R}^{*}}\right\|_{L^{\infty}\left(\mathbb{C}_{j}^{\circ}\right)}\right\} d_{\mathbb{R}, 1, S}\left(\pi \cap \mathbb{C}_{j}\right) .
$$

Thus we obtain

$$
\begin{aligned}
& \sum_{i \in Q_{*}}\left(\left(\frac{d_{\mathbb{R}, 1, S}^{(i)}\left(\pi_{i}^{-}\right)}{d_{\mathbb{R}, 1, S}(\pi)}-1\right)^{+}\right)^{2} \mathbf{1}_{\mathcal{A}_{i}} \\
& \leq d_{\mathbb{R}, 1, S}(\pi)^{-2} \sum_{i \in Q_{*}}\left(\sum_{j \in \mathcal{D}(i)}\left(\exp \left\{\gamma\left\|h_{\mathbb{R}^{*}}-h_{\mathbb{R}^{*}}^{(i)}\right\|_{L^{\infty}\left(\mathbb{C}_{j}^{\circ}\right)}\right\}-1\right) d_{\mathbb{R}, 1, S}\left(\pi \cap \mathbb{C}_{j}\right)\right)^{2} \mathbf{1}_{\mathcal{A}_{i}} \\
& =d_{\mathbb{R}, 1, S}(\pi)^{-2} \sum_{j_{1}, j_{2} \in Q_{\circ}}\left[d_{\mathbb{R}, 1, S}\left(\pi \cap \mathbb{C}_{j_{1}}\right) d_{\mathbb{R}, 1, S}\left(\pi \cap \mathbb{C}_{j_{2}}\right) \sum_{\substack{i \in Q_{*} \\
\mathcal{D}(i) \ni j_{1}, j_{2}}} \mathbf{1}_{\mathcal{A}_{i}} \prod_{j \in\left\{j_{1}, j_{2}\right\}}\left(\exp \left\{\gamma\left\|h_{\mathbb{R}^{*}}-h_{\mathbb{R}^{*}}^{(i)}\right\|_{\left.L^{\infty}\left(\mathbb{C}_{j}^{\circ}\right)\right\}}-1\right)\right]\right. \\
& \leq d_{\mathbb{R}, 1, S}(\pi)^{-2}\left(\sum_{j \in Q_{\circ}} d_{\mathbb{R}, 1, S}\left(\pi \cap \mathbb{C}_{j}\right)\right) \\
& \cdot \max _{j_{2} \in Q_{\circ}}\left[\sum_{j_{1} \in Q_{\circ}}\left(d_{\mathbb{R}, 1, S}\left(\pi \cap \mathbb{C}_{j_{1}}\right) \sum_{\substack{i \in Q_{*} \\
\mathcal{D}(i) \ni j_{1}, j_{2}}} \mathbf{1}_{\mathcal{A}_{i}} \prod_{j \in\left\{j_{1}, j_{2}\right\}}\left(\exp \left\{\gamma\left\|h_{\mathbb{R}^{*}}-h_{\mathbb{R}^{*}}^{(i)}\right\|_{\left.L^{\infty}\left(\mathbb{C}_{j}^{\circ}\right)\right\}}\right\}\right)\right],\right.
\end{aligned}
$$

where the equality comes from expanding out the square and switching the order of summation, and the inequality is the $\ell^{1}-\ell^{\infty}$ Hölder inequality. Now we have that

$$
\sum_{j \in Q_{0}} d_{\mathbb{R}, 1, S}\left(\pi \cap \mathbb{C}_{j}\right) \leq \sum_{j \in Q_{\circ}} \sum_{\substack{1 \leq m \leq M \\ \pi_{m} \cap \mathbb{C}_{j} \neq \emptyset}} d_{\mathbb{R}, 1, S}\left(\pi_{m}\right) \leq \sum_{1 \leq m \leq M} \#\left\{j \in Q_{\circ}: \pi_{m} \cap \mathbb{C}_{j} \neq \emptyset\right\} \cdot d_{\mathbb{R}, 1, S}\left(\pi_{m}\right) \leq C d_{\mathbb{R}, 1, S}(\pi),
$$

in which the last inequality is by (5.10) and (5.6). Also, we have

$$
\mathbf{1}_{\mathcal{A}_{i}} d_{\mathbb{R}, 1, S}\left(\pi \cap \mathbb{C}_{j_{1}}\right) \leq \mathbf{1}_{\mathcal{A}_{i}} d_{\mathbb{R}^{*}, \mathbb{A}_{j_{1}}, 1, S} \text { (around) } \leq \exp \left\{\gamma \frac{\operatorname{dist}\left(\mathbb{C}_{i}, \mathbb{C}_{j_{1}}^{\circ}\right)}{S}\right\} d_{\left(3 \mathbb{E}_{i}\right)^{*}, \mathbb{A}_{j_{1}}, K^{-\gamma \theta_{0}, S}}(\text { around }) .
$$

Here, the first inequality is because the part of $\pi$ intersecting $\mathbb{C}_{j}$ could be replaced by a circuit around the annulus $\mathbb{A}_{j}$ to produce a new crossing, so $\pi \cap \mathbb{C}_{j}$ must have a smaller LGD length than the annular circuit around $\mathbb{A}_{j}$ by the optimality of $\pi$. (A similar, but not quite identical, argument was used to derive (5.14).) The second inequality in (5.54) is by applying (2.29) and (2.41) using the coarse field bound (5.16).

Plugging (5.53) and (5.54) into the right-hand side of (5.52), we obtain

$$
\begin{aligned}
& \sum_{i \in Q_{*}}\left(\left(\frac{d_{\mathbb{R}, 1, S}^{(i)}\left(\pi_{i}^{-}\right)}{d_{\mathbb{R}, 1, S}(\pi)}-1\right)^{+}\right)^{2} \mathbf{1}_{\mathcal{A}_{i}} \leq\left(\frac{\max _{j \in Q_{\circ}} d_{\left(3 \mathbb{E}_{i}\right)^{*}, \mathbb{A}_{j_{1}}, K^{-\gamma \theta_{0}, S}}(\text { around })}{d_{\mathbb{R}, 1, S}(\pi)}\right) . \\
& \cdot \max _{j_{2} \in Q_{\circ}} \sum_{\substack{j_{1} \in Q_{\circ} \\
i \in Q_{*} \\
\mathcal{D}(i) \ni j_{1}, j_{2}}} \exp \left\{\gamma \frac{\operatorname{dist}\left(\mathbb{C}_{i}, \mathbb{C}_{j_{1}}^{\circ}\right)}{S}\right\} \prod_{j \in\left\{j_{1}, j_{2}\right\}}\left(\exp \left\{\gamma\left\|h_{\mathbb{R}^{*}}-h_{\mathbb{R}^{*}}^{(i)}\right\|_{\left.L^{\infty}\left(\mathbb{C}_{j}^{\circ}\right)\right\}}-1\right) .\right.
\end{aligned}
$$


We can bound the sum on the right-hand side by

$$
\begin{aligned}
\sum_{\substack{j_{1} \in Q_{\circ} \\
i \in Q_{*} \\
\mathcal{D}(i) \ni j_{1}, j_{2}}} \exp \left\{\gamma \frac{\operatorname{dist}\left(\mathbb{C}_{i}, \mathbb{C}_{j_{1}}^{\circ}\right)}{S}\right\} \prod_{j \in\left\{j_{1}, j_{2}\right\}}\left(\operatorname { e x p } \left\{\gamma\left\|h_{\mathbb{R}^{*}}-h_{\mathbb{R}^{*}}^{(i)}\right\|_{\left.\left.L^{\infty}\left(\mathbb{C}_{j}^{\circ}\right)\right\}-1\right)}\right.\right. \\
\leq \sum_{\substack{j_{1} \in Q_{\circ} \\
i \in Q_{*} \\
\mathcal{D}(i) \ni j_{1}, j_{2}}} \exp \left\{\gamma \frac{\operatorname{dist}\left(\mathbb{C}_{i}, \mathbb{C}_{j_{1}}^{\circ}\right)}{S}\right\} \prod_{j \in\left\{j_{1}, j_{2}\right\}}\left(\exp \left\{\gamma G^{*} \exp \left\{-\frac{\operatorname{dist}_{\mathbb{E}}\left(\mathbb{C}_{i}, \mathbb{C}_{j}^{\circ}\right)^{2}}{C S^{2}}\right\}\right\}-1\right) \leq \mathrm{e}^{C \gamma G^{*}},
\end{aligned}
$$

where $G^{*}$ is as in (5.46) and the last inequality is a simple estimate on the sum. Plugging (5.56) into (5.55), we and applying Hölder's inequality and Lemma 5.9, we obtain (5.51).

\subsubsection{The close fine field effect}

Now we deal with the part of the path that is close to the part of the white noise being resampled: the last term of (5.20). Here, when we resample, we replace the part of the path that is close to the resampled box by a circuit around an annulus surrounding the box. The "fine field" has been totally changed in the resampling, so there is nothing to be gained by keeping track of it. However, the "coarse field" should only be changed by a small, smooth difference, and we want to keep track of this so that the sum of all the changes will be bounded by the total weight of the original path. Thus we define the notation (recalling that $\theta_{0}$ was fixed in (2.12))

$$
F_{i}^{\uparrow}=\max _{x \in \mathbb{E}_{i}^{\circ}} h_{\mathbb{R}^{*}: \mathbb{E}_{i}^{*}}^{(i)}(x), \quad F_{i}^{\downarrow}=\min _{x \in \mathbb{E}_{i}^{\circ}} h_{\mathbb{R}^{*}: \mathbb{E}_{i}^{*}}(x),
$$

The difference between $F_{i}^{\uparrow}$ and $F_{i}^{\downarrow}$ represents the "change in the coarse field," and it will be of order 1, as we show in the next two lemmas.

Lemma 5.11. For every $\lambda>0$, we have a constant $C<\infty$, depending on $\lambda$, so that

$$
\mathbf{E} \exp \left\{\lambda \sup _{x \in \mathbb{E}_{i}^{\circ}}\left|h_{\mathbb{R}^{*}: \mathbb{E}_{i}^{*}}^{(i)}(x)-h_{\mathbb{R}^{*}: \mathbb{E}_{i}^{*}}(x)\right|\right\} \leq C .
$$

Proof. Inequality (5.45) of Lemma 5.9 implies that for any $\lambda>0$ there is a $C<\infty$, depending only on $\lambda$, so that

$$
\mathbf{E} \exp \left\{\lambda \sup _{x \in \mathbb{E}_{i}^{*} \backslash \mathbb{E}_{i}^{\circ}}\left|h_{\mathbb{R}^{*}}^{(i)}(x)-h_{\mathbb{R}^{*}}(x)\right|\right\} \leq C .
$$

By the maximum principle for harmonic functions, we have that

$$
\sup _{x \in \mathbb{E}_{i}^{\circ}}\left|h_{\mathbb{R}^{*}: \mathbb{E}_{i}^{*}}^{(i)}(x)-h_{\mathbb{R}^{*}: \mathbb{E}_{i}^{*}}(x)\right| \leq \sup _{x \in \mathbb{E}_{i}^{*} \backslash \mathbb{E}_{i}^{\circ}}\left|h_{\mathbb{R}^{*}}^{(i)}(x)-h_{\mathbb{R}^{*}}(x)\right|,
$$

by which we obtain (5.58).

Lemma 5.12. For any $B>0$ and $A<\infty$ we have a $C>0$ so that

$$
\mathbf{E e}^{\gamma B\left(F_{i}^{\uparrow}-F_{i}^{\downarrow}\right)} \leq C K^{1 / A}
$$


Proof. We have that

$$
\begin{aligned}
F_{i}^{\uparrow}-F_{i}^{\downarrow} & =\max _{x \in \mathbb{E}_{i}^{\circ}} h_{\mathbb{R}^{*}: \mathbb{E}_{i}^{*}}^{(i)}(x)-\min _{x \in \mathbb{E}_{i}^{\circ}} h_{\mathbb{R}^{*}: \mathbb{E}_{i}^{*}}(x)=\max _{x, y \in \mathbb{E}_{i}^{\circ}}\left(h_{\mathbb{R}^{*}: \mathbb{E}_{i}^{o}}^{(i)}(x)-h_{\mathbb{R}^{*}: \mathbb{E}_{i}^{*}}(y)\right) \\
& \leq \max _{x, y \in \mathbb{E}_{i}^{\circ}}\left|h_{\mathbb{R}^{*}: \mathbb{E}_{i}^{*}}(x)-h_{\mathbb{R}^{*}: \mathbb{E}_{i}^{*}}(y)\right|+\max _{x \in \mathbb{E}_{i}^{\circ}}\left|h_{\mathbb{R}^{*}: \mathbb{E}_{i}^{*}}^{(i)}(x)-h_{\mathbb{R}^{*}: \mathbb{E}_{i}^{*}}(x)\right| .
\end{aligned}
$$

To bound the first term of (5.60), we note that by Lemma 2.9, we have for any $\lambda>0$ that there is a $C$ depending on $\lambda$ so that

$$
\mathbf{E} \exp \left\{\lambda \max _{x, y \in \mathbb{E}_{i}^{\circ}}\left|h_{\mathbb{R}^{*}: \mathbb{E}_{i}^{*}}(x)-h_{\mathbb{R}^{*}: \mathbb{E}_{i}^{*}}(y)\right|\right\} \leq C .
$$

Therefore, by Jensen's inequality, we have for any $A>0$ that

$$
\begin{gathered}
\mathbf{E} \exp \left\{\lambda \max _{i \in Q_{*}} \max _{x, y \in \mathbb{E}_{i}^{\circ}}\left|h_{\mathbb{R}^{*}: \mathbb{E}_{i}^{*}}(x)-h_{\mathbb{R}^{*}: \mathbb{E}_{i}^{*}}(y)\right|\right\} \leq\left(\mathbf{E} \exp \left\{A \lambda \max _{i \in Q_{*}} \max _{x, y \in \mathbb{E}_{i}^{\circ}}\left|h_{\mathbb{R}^{*}: \mathbb{E}_{i}^{*}}(x)-h_{\mathbb{R}^{*}: \mathbb{E}_{i}^{*}}(y)\right|\right\}\right)^{1 / A} \\
\leq\left(\sum_{i \in Q_{*}} \mathbf{E} \exp \left\{A \lambda \max _{x, y \in \mathbb{E}_{i}^{\circ}}\left|h_{\mathbb{R}^{*}: \mathbb{E}_{i}^{\circ}}(x)-h_{\mathbb{R}^{*}: \mathbb{E}_{i}^{\circ}}(y)\right|\right\}\right)^{1 / A} \leq C\left|Q_{*}\right|^{1 / A} \leq C K^{2 / A},
\end{gathered}
$$

for some $C$ depending on $A$ and $\lambda$. Considering the second term of (5.60), we note that, by Lemma 5.11, we have

$$
\mathbf{E} \exp \left\{\lambda \max _{x \in \mathbb{E}_{i}^{\circ}}\left|h_{\mathbb{R}^{*}: \mathbb{E}_{i}^{*}}^{(i)}(x)-h_{\mathbb{R}^{*}: \mathbb{E}_{i}^{*}}(x)\right|\right\} \leq C
$$

for some $C$ depending on $\lambda$. Bounding the exponential moments of the first and second terms of (5.60) using (5.61) and (5.62) respectively, and then using Young's inequality, yields (5.59).

Now we define

$$
H_{i}=\frac{d^{(i)}\left(3 \mathbb{E}_{i}\right)^{*}, \mathbb{A}_{i}, \mathrm{e}^{-\gamma F_{i}^{\uparrow}, S}}{d_{\mathbb{E}_{i}, \mathrm{e}^{-\gamma F_{i}^{\downarrow}} \downarrow}\left(\min ; S / \operatorname{diam}_{\mathrm{E}}\left(\mathbb{E}_{i}\right)\right.}, \quad H=\max _{i \in Q_{\circ}} H_{i},
$$

representing the cost of replacing a geodesic across a box with respect the original fine field with a circuit around the box with respect to the resampled fine field. The choices of $\delta$ (as $\mathrm{e}^{-\gamma F_{i}^{\uparrow}}$ and $\mathrm{e}^{-\gamma F_{i}^{\downarrow}}$ ) in the definition of $H_{i}$ represent the worst-case contributions of the coarse field, which we bounded in the previous lemma. We now bound the sum of all of the "replacement" annuli, divided by the total crossing length, in terms of $H$.

Lemma 5.13. There is a constant $C<\infty$ so that

$$
\frac{\sum_{\substack{i \in Q_{*} \\ \mathcal{M}(i) \neq \emptyset}} d_{\mathbb{R}^{*}, \mathbb{A}_{i}, 1, S}^{(i)}(\text { around })}{d_{\mathbb{R}, 1, S}(\mathrm{~L}, \mathrm{R})} \leq C H .
$$

Proof. By (2.41) and (2.42) of Proposition 2.38, we have

$$
\begin{aligned}
& d_{\mathbb{R}^{*}, \mathbb{A}_{i}, 1, S}^{(i)}(\text { around }) \leq d_{\left(3 \mathbb{E}_{i}\right)^{*}, \mathbb{A}_{i}, \mathrm{e}^{-\gamma F_{i}^{\uparrow}, S}}^{(i)} \text { (around), } \\
& d_{\mathbb{R}^{*}, \mathbb{E}_{i}, 1, S}\left(x_{m-1}, x_{m}\right) \geq d_{\mathbb{E}_{i}, \mathrm{e}^{-\gamma F_{i}, S}}\left(x_{m-1}, x_{m}\right),
\end{aligned}
$$


where we recall that $x_{m-1}, x_{m}$ are the endpoints of $\pi_{m}$ as defined in Subsection 5.2. Thus we have

$$
\begin{aligned}
& d_{\mathbb{R}^{*}, \mathbb{A}_{i}, 1, S}^{(i)} \text { (around) } \leq d_{\left(3 \mathbb{E}_{i}\right)^{*}, \mathbb{A}_{i}, \mathrm{e}^{-\gamma F_{i}^{\uparrow}, S}}^{(i)} \text { (around) } \leq H_{i} d_{\mathbb{E}_{i}, \mathrm{e}^{-\gamma F_{i}} \downarrow}\left(x_{m-1}, x_{m}\right) \\
& \leq H_{i} d_{\mathbb{R}^{*}, \mathbb{E}_{i}, 1, S}\left(x_{m-1}, x_{m}\right),
\end{aligned}
$$

where the last inequality is by (2.41) of Proposition 2.38. Also, we have a constant $C$ so that

$$
\sum_{\substack{i \in Q_{*} \\ m \in \mathcal{M}(i)}} d_{\mathbb{R}^{*}, \mathbb{E}_{i}, 1, S}\left(x_{m-1}, x_{m}\right)=\sum_{\substack{i \in Q_{*} \\ m \in \mathcal{M}(i)}} d_{\mathbb{R}, 1, S}\left(x_{m-1}, x_{m}\right) \leq C \sum_{m=1}^{M} d_{\mathbb{R}, 1, S}\left(x_{m-1}, x_{m}\right)=C d_{\mathbb{R}, 1, S}(\mathrm{~L}, \mathrm{R}),
$$

where the first equality is by (5.12), the inequality is by (5.11), and the second equality is by (5.6). Therefore, for each $i \in Q$, if we set $m(i)=\min \mathcal{M}(i)$ (or just an arbitrary element of $\mathcal{M}(i)$ picked in some other way), then we have

$$
\sum_{\substack{i \in Q_{*} \\ \mathcal{M}(i) \neq \emptyset}} \frac{d_{\mathbb{R}^{*}, \mathbb{A}_{i}, 1, S}^{(i)}(\text { around })}{d_{\mathbb{R}, 1, S}(\mathrm{~L}, \mathrm{R})} \leq H \sum_{i \in Q_{*}} \frac{d_{\mathbb{R}^{*}, \mathbb{E}_{i}, 1, S}\left(x_{m(i)-1}, x_{m(i)}\right)}{d_{\mathbb{R}, 1, S}(\mathrm{~L}, \mathrm{R})} \leq H \frac{\sum_{i \in Q_{*}} \sum_{m \in \mathcal{M}(i)} d_{\mathbb{R}^{*}, \mathbb{E}_{i}, 1, S}\left(x_{m-1}, x_{m}\right)}{d_{\mathbb{R}, 1, S}(\mathrm{~L}, \mathrm{R})} \leq C H,
$$

where the first inequality is by (5.64) and the third is by (5.65). This proves (5.63).

Lemma 5.14. For any $B<\infty$ and $A<\infty$ we have $a C<$ so that

$$
\mathbf{E}\left[\left(\sum_{\substack{i \in Q_{*} \\ \mathcal{M}(i) \neq \emptyset}} \frac{\mathbf{1}_{\mathcal{A}_{i}} d_{\mathbb{R}^{*}, \mathbb{A}_{i}, 1, S}^{(i)}(\text { around })}{d_{\mathbb{R}, 1, S}(\mathrm{~L}, \mathrm{R})}\right)^{B}\right] \leq C K^{2} \bar{\chi}_{K^{\eta} S}^{B}
$$

Proof. First we fix $i \in Q_{*}$. We have, by Hölder's inequality,

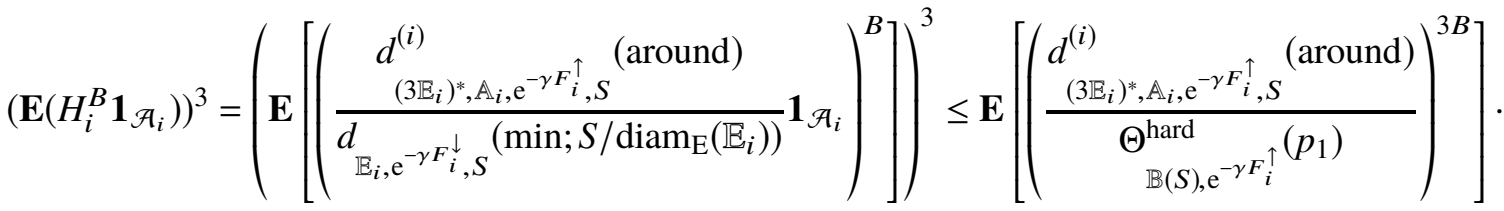

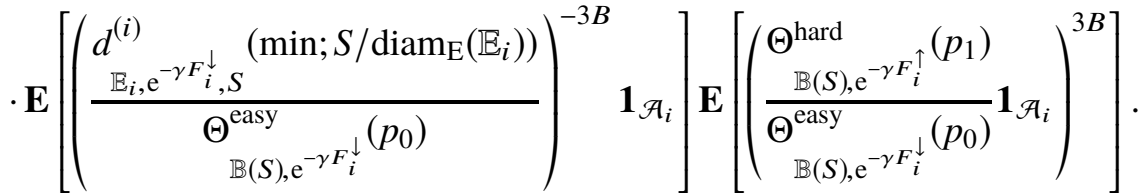

We bound each of the factors in turn. First we compute

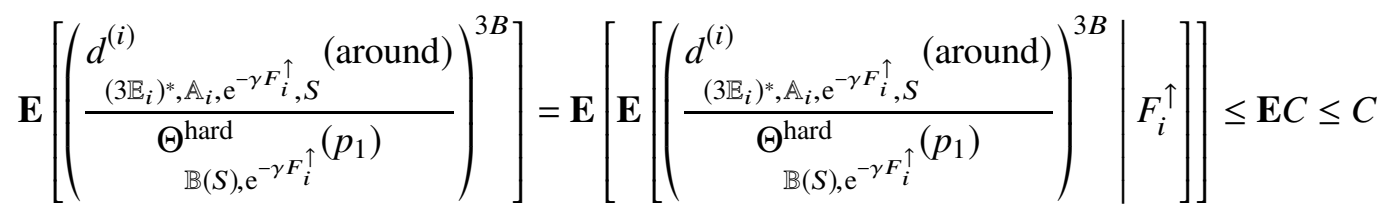

by Corollary 4.9 and (2.32) of Lemma 2.23. For the second two terms, we first note that, on the event $\left\{F_{i}^{\downarrow} \leq \theta_{0} \log K\right\} \supset\left\{F_{i}^{\uparrow} \leq \theta_{0} \log K\right\} \supset \mathcal{A}_{i}$, we have

$$
\frac{2 \gamma F_{i}^{\downarrow}}{4+\gamma^{2}} \leq \frac{2 \gamma F_{i}^{\uparrow}}{4+\gamma^{2}} \leq \frac{2 \gamma \theta_{0} \log K}{4+\gamma^{2}} \leq \eta \log K,
$$


where the first inequality is by the definitions (5.57) of $F_{i}^{\downarrow}$ and $F_{i}^{\uparrow}$, the second inequality is by the definition (5.17) of the event $\mathcal{A}_{i}$, and the third inequality is by the definition (2.12) of $\eta$. Thus we have

$$
\begin{aligned}
& \mathbf{E}\left[\left(\frac{d_{\mathbb{E}_{i}, \mathrm{e}^{-\gamma F_{i, S}^{\downarrow}}}\left(\min ; S / \operatorname{diam}_{\mathbb{E}}\left(\mathbb{E}_{i}\right)\right)}{\Theta_{\mathbb{B}(S), \mathrm{e}^{-\gamma F_{i}^{\downarrow}}}^{\text {easy }}\left(p_{0}\right)}\right)^{-3 B} \mathbf{1}_{\mathcal{A}_{i}}\right] \\
& \leq \mathbf{E}\left[\mathbf{E}\left[\left(\frac{d_{\mathbb{E}_{i}, \mathrm{e}^{-\gamma F_{i, S}^{\downarrow}}}\left(\min ; S / \operatorname{diam}_{\mathrm{E}}\left(\mathbb{E}_{i}\right)\right)}{\Theta_{\mathbb{B}(S), \mathrm{e}^{-\gamma F_{i}^{\downarrow}}}^{\text {easy }}\left(p_{0}\right)}\right)^{-3 B} \mathbf{1}_{\left\{F_{i}^{\downarrow} \leq \theta_{0} \log K\right\}} \mid F_{i}^{\downarrow}\right]\right]
\end{aligned}
$$

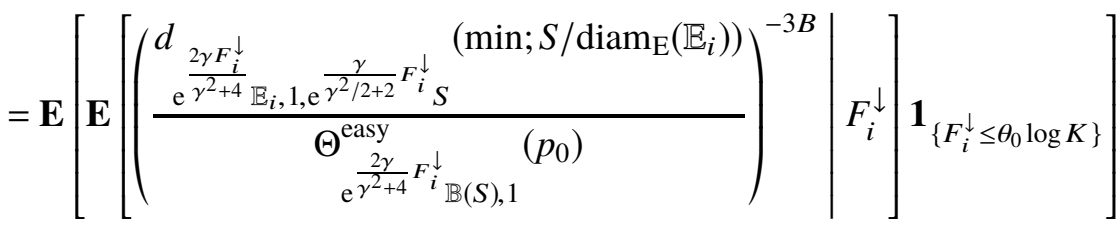

$$
\begin{aligned}
& \leq C \mathbf{E}\left[\bar{\chi}_{\mathrm{e}^{\frac{2 \gamma}{\gamma^{2}+4} F_{i}}}^{3 B} \mathbf{1}_{\left\{F_{i}^{\downarrow} \leq \theta_{0} \log K\right\}}\right] \leq C \mathbf{E} \bar{\chi}_{K^{\eta} S}^{3 B}=C \bar{\chi}_{K^{\eta} S}^{3 B},
\end{aligned}
$$

where the second equality is by Proposition 2.35, the first inequality is by Proposition 4.7, and the second inequality is by (5.69).

For the third term in (5.67), put $K^{*}=\exp \left\{\frac{2 \gamma F_{i}^{\uparrow}}{4+\gamma^{2}}\right\}$. On the event $\mathcal{A}_{i}$, by (5.69) we have $K^{*} \leq K^{\eta}$. Then we can write, using (2.28) twice, recalling Definition 4.3, and applying (2.29), that

$$
\begin{aligned}
\mathbf{1}_{\mathcal{A}_{i}} \Theta_{\mathbb{B}(S), \mathrm{e}^{-\gamma F_{i}^{\uparrow}}}^{\text {hard }}\left(p_{1}\right)=\mathbf{1}_{\mathcal{A}_{i}} \Theta_{\mathbb{B}\left(K^{*} S\right)}^{\text {hard }}\left(p_{1}\right) \leq \mathbf{1}_{\mathcal{A}_{i}} \bar{\chi}_{K^{\eta} S} \Theta_{\mathbb{B}\left(K^{*} S\right)}^{\text {easy }}\left(p_{0}\right) & =\mathbf{1}_{\mathcal{A}_{i}} \bar{\chi}_{K^{\eta} S} \Theta_{\mathbb{B}(S), \mathrm{e}^{-\gamma F_{i}^{\uparrow}}}^{\text {easy }}\left(p_{0}\right) \\
& \leq \mathbf{1}_{\mathcal{A}_{i}} \bar{\chi}_{K^{\eta} S} \mathrm{e}^{\gamma\left(F_{i}^{\uparrow}-F_{i}^{\downarrow}\right)} \Theta^{\text {easy }}{\mathbb{B}(S), \mathrm{e}^{-\gamma F_{i}^{\downarrow}}}^{\left(p_{0}\right) .}
\end{aligned}
$$

Therefore, we have

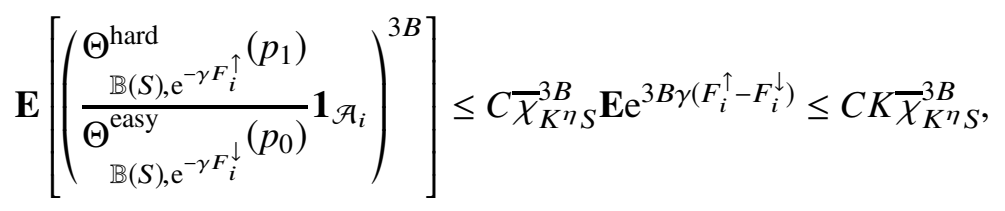

where the last inequality is by Lemma 5.12. Plugging (5.68), (5.70), and (5.71) into (5.67) yields

$$
\mathbf{E}\left(H_{i}^{B} \mathbf{1}_{\mathcal{A}_{i}}\right) \leq C K \bar{\chi}_{K^{\eta} S}^{B} .
$$

Then we can write

$$
\mathbf{E}\left[\left(\sum_{\substack{i \in Q_{*} \\ \mathcal{M}(i) \neq \emptyset}} \frac{d_{\mathbb{R}^{*}, \mathbb{A}_{i}, 1, S}^{(\text {around })}}{d_{\mathbb{R}, 1, S}(\mathrm{~L}, \mathrm{R})} \mathbf{1}_{\mathcal{A}_{i}}\right)^{B}\right] \leq C \mathbf{E}\left(H^{B} \mathbf{1}_{\mathcal{H}_{i}}\right) \leq C \sum_{i \in Q_{*}} \mathbf{E}\left(H_{i}^{B} \mathbf{1}_{\mathcal{A}_{i}}\right) \leq C K^{2} \bar{\chi}_{K^{\eta} S}^{B},
$$

where the first inequality is by Lemma 5.13 and the Cauchy-Schwarz inequality and the second inequality uses (5.72) and the fact that $\left|Q_{*}\right| \leq C K^{2}$. But the last display is (5.66). 


\subsubsection{The maximum small box crossing weight}

We now show that the maximum annular circuit distance is much smaller (for large $K$ ) than the crossing quantile of the large rectangle $\mathbb{R}$. This will be used for bounding the middle factor of (5.51) as well as the first factor in the last term of (5.20). Here we crucially use that $\gamma<2$, as this means that even after considering the coarse field, subboxes "look" smaller than the large box.

Lemma 5.15. Define notation as in the statement of Lemma 5.13. Suppose that $T_{0} \leq K^{\eta} S$, where $T_{0}$ is as in Proposition 4.4. For any $B \geq 0$ there is a $c>0$ so that

$$
\mathbf{E}\left[\left(\max _{i \in Q_{*}} d_{\left(3 \mathbb{E}_{i}\right)^{*}, \mathbb{A}_{i}, K^{-\gamma \theta_{0}}}^{(\text {around })}\right)^{B}\right], \mathbf{E}\left[\left(\max _{i \in Q_{*}} d_{\left(3 \mathbb{E}_{i}\right)^{*}, \mathbb{A}_{i}, K^{-\gamma \theta_{0}}}(\text { around })\right)^{B}\right] \leq C \bar{\chi}_{K^{\eta} S}^{B} K^{-c} \Theta_{\mathbb{B}(K S)}^{\text {easy }}\left(p_{0}\right)^{B},
$$

Proof. We have

$$
\mathbf{E}\left[\left(\max _{i \in Q_{*}} d_{\left(3 \mathbb{E}_{i}\right)^{*}, \mathbb{A}_{i}, K^{-\gamma \theta_{0}}}^{(i)}(\text { around })\right)^{B}\right] \leq \sum_{i \in Q_{*}} \mathbf{E} d_{\left(3 \mathbb{E}_{i}\right)^{*}, \mathbb{A}_{i}, K^{-\gamma \theta_{0}}}^{(i)}(\text { around })^{B} \leq C\left|Q_{*}\right|^{\frac{1}{2}} \Theta_{\mathbb{B}\left(K^{\eta} S\right)}^{\text {hard }}\left(p_{1}\right)^{B},
$$

where the second inequality is by (2.28) and Corollary 4.9, and in the last expression the constant $C$ depends on $B$. (Recall that $p_{1}$ is fixed as in Proposition 4.2.) On the other hand, we have that

$$
\Theta_{\mathbb{B}\left(K^{\eta} S\right)}^{\text {hard }}\left(p_{1}\right) \leq \bar{\chi}_{K^{\eta} S} \Theta_{\mathbb{B}\left(K^{\eta} S\right)}^{\text {easy }}\left(p_{0}\right) \leq C \bar{\chi}_{K^{\eta} S} K^{-c(1-\eta)} \Theta_{\mathbb{B}(K S)}^{\text {easy }}\left(p_{0}\right),
$$

where the first inequality is by Definition 4.3 and the second is by (4.8). Here we use the assumption that $K^{\eta} S \geq T_{0}$. Plugging the last inequality into (5.74) yields

$$
\begin{aligned}
\mathbf{E}\left[\left(\max _{i \in Q_{*}} d_{\left(3 \mathbb{E}_{i}\right)^{*}, \mathbb{A}_{i}, K^{-\gamma \theta_{0}}}^{\left.(\text {around }))^{B}\right]}\right.\right. & \leq C \bar{\chi}_{K^{\eta} S}^{B}\left|Q_{*}\right|^{1 / 2} K^{-B c(1-\eta)} \Theta_{\mathbb{B}(K S)}^{\text {easy }}\left(p_{0}\right)^{B} \\
& \leq C \bar{\chi}_{K^{\eta} S}^{B} K^{1-B c(1-\eta)} \Theta_{\mathbb{B}(K S)}^{\text {easy }}\left(p_{0}\right)^{B} .
\end{aligned}
$$

Choosing $B$ large enough so that $-c^{\prime}:=1-B c(1-\eta)<0$, this becomes

$$
\mathbf{E}\left[\left(\max _{i \in Q_{*}} d_{\left(3 \mathbb{E}_{i}\right)^{*}, \mathbb{A}_{i}, K^{-\gamma \theta_{0}}}^{(i)}(\text { around })\right)^{B}\right] \leq C \bar{\chi}_{K^{\eta} S^{\prime}}^{B} K^{-c^{\prime}} \Theta_{\mathbb{B}(K S)}^{\text {easy }}\left(p_{0}\right)^{B},
$$

which is half of (5.73). The other half follows in the same way, noting that (5.74) uses nothing about the correlations between the $d_{\left(3 \mathbb{E}_{i}\right)^{*}, \mathbb{A}_{i}, K^{-\gamma \theta_{0}}}^{(i)}$ (around)s for varying $i$.

\subsubsection{The effect of requiring small balls}

Our Efron-Stein argument required restricting the balls used to cover the path to be of size at most $S$. This was important for our percolation argument, because otherwise a single ball could be used to cover the path in potentially very many of the $\mathbb{C}_{i} \mathrm{~s}$. However, the effect of this requirement should be negligible, because at large scales, we do not expect large balls to be used: recall from (2.14) that the LQG measure has all negative moments. The next lemma quantifies this intuition.

Lemma 5.16. Suppose $K S \geq T_{0}$ (defined as in Proposition 4.4). There are constants $C<\infty$ and $c>0$ so that

$$
\mathbf{E}\left(\log \frac{d_{\mathbb{R}, 1, S}(\mathrm{~L}, \mathrm{R})}{d_{\mathbb{R}}(\mathrm{L}, \mathrm{R})}\right)^{2} \leq C K^{C} S^{-c}
$$


Proof. By (2.31), we have

$$
\log \frac{d_{\mathbb{R}, 1, S}(\mathrm{~L}, \mathrm{R})}{d_{\mathbb{R}}(\mathrm{L}, \mathrm{R})} \leq \log \left(1+\frac{K^{3}}{d_{\mathbb{R}}(\mathrm{L}, \mathrm{R})}\right) \leq \frac{K^{3}}{d_{\mathbb{R}}(\mathrm{L}, \mathrm{R})}
$$

almost surely. Therefore, we have, as long as $K S \geq T_{0}$, that

$$
\mathbf{E}\left[\left(\log \frac{d_{\mathbb{R}, 1, S}(\mathrm{~L}, \mathrm{R})}{d_{\mathbb{R}}(\mathrm{L}, \mathrm{R})}\right)^{2}\right] \leq K^{6} \mathbf{E} d_{\mathbb{R}}(\mathrm{L}, \mathrm{R})^{-2} \leq C K^{6} \bar{\chi}_{K S}\left(\frac{K S}{T_{0}}\right)^{-c} \Theta_{\mathbb{B}\left(T_{0}\right)}^{\text {easy }}(p)^{-2} \leq C K^{C} S^{-c} .
$$

with the second inequality by Proposition 4.7, the third by Proposition 4.4, and the last by (4.7).

\subsubsection{The large coarse field error term}

Here we bound the term of (5.20) corresponding to the event that the coarse field is extraordinarily large.

Lemma 5.17. There are constants $C<\infty$ and $c>0$ so that

$$
\sum_{i \in Q_{*}} \mathbf{E}\left[\left(\left(\log \frac{d_{\mathbb{R}, 1, S}^{(i)}(\mathrm{L}, \mathrm{R})}{d_{\mathbb{R}, 1, S}(\mathrm{~L}, \mathrm{R})}\right)^{+}\right)^{2} \mathbf{1}_{\mathcal{A}_{i}^{c}}\right] \leq C K^{-c} \bar{\chi}_{S}^{3}
$$

Proof. Whenever $\alpha, \beta \in(1, \infty)$ and $1 / \alpha+1 / \beta=1$, we have

$$
\left.\mathbf{E}\left[\left(\log \frac{d_{\mathbb{R}, 1, S}^{(i)}(\mathrm{L}, \mathrm{R})}{d_{\mathbb{R}, 1, S}(\mathrm{~L}, \mathrm{R})}\right)^{+}\right)^{2} \mathbf{1}_{\mathcal{A}_{i}^{c}}\right] \leq 4\left[\mathbf{P}\left(\mathcal{A}_{i}^{c}\right)\right]^{1 / \alpha}\left[\mathbf{E}\left(\left(\log \frac{d_{\mathbb{R}, 1, S}^{(i)}(\mathrm{L}, \mathrm{R})}{d_{\mathbb{R}, 1, S}(\mathrm{~L}, \mathrm{R})}\right)^{+}\right)^{2 \beta}\right]^{1 / \beta}
$$

by Hölder's inequality. By Lemma 5.4, $\mathbf{P}\left(\mathcal{A}_{i}^{c}\right) \leq K^{-\theta_{0}^{2} / 2}$, so

$$
\sum_{i \in Q_{*}}\left[\mathbf{P}\left(\mathcal{A}_{i}^{c}\right)\right]^{1 / \alpha} \leq K^{2-\frac{\theta_{0}^{2}}{2 \alpha}}
$$

and the exponent $-2 c:=2-\frac{\theta_{0}^{2}}{2 \alpha}$ is negative so long as $\alpha$ is chosen sufficiently close to 1 . Also, we have constants $A, C>0$ so that by Proposition 4.8 and Proposition 4.6,

$$
\mathbf{E} d_{\mathbb{R}, 1, S}^{(i)}(\mathrm{L}, \mathrm{R}) \leq C K^{A} \Theta_{\mathbb{B}(S)}^{\text {hard }}\left(p_{1}\right)
$$

and by Proposition 4.7 and Proposition 4.4,

$$
\mathbf{E}\left[\left(d_{\mathbb{R}, 1, S}(\mathrm{~L}, \mathrm{R})\right)^{-1}\right] \leq C \bar{\chi}_{S} K^{A} \Theta_{\mathbb{B}(S)}^{\text {easy }}\left(p_{0}\right)^{-1} .
$$

For any $b>0$, we have a constant $C=C(b)$ so that $(\log x)^{+} \leq C x^{b}$ for all $x>0$. Using this fact, the CauchySchwarz and Jensen inequalities, and (5.77) and (5.78), we obtain in particular that as long as $4 b \beta \leq 1$, for each $i \in Q_{*}$,

$$
\left[\mathbf{E}\left(\left(\log \frac{d_{\mathbb{R}, 1, S}^{(i)}(\mathrm{L}, \mathrm{R})}{d_{\mathbb{R}, 1, S}(\mathrm{~L}, \mathrm{R})}\right)^{+}\right)^{2 \beta}\right]^{1 / \beta} \leq C\left[\left(K^{A} \Theta_{\mathbb{B}(S)}^{\mathrm{hard}}\left(p_{1}\right)\right)\left(\bar{\chi}_{S} K^{A} \Theta_{\mathbb{B}(S)}^{\text {easy }}\left(p_{0}\right)^{-1}\right)\right]^{4 b}
$$


Taking $b$ so small that $4 \beta b \leq 1,8 A b<c$ and $b \leq 1 / 4$, we obtain from (5.75), (5.76) and (5.79) that

$$
\begin{array}{r}
\sum_{i \in Q_{*}} \mathbf{E}\left[\left(\left(\log \frac{d_{\mathbb{R}, 1, S}^{(i)}(\mathrm{L}, \mathrm{R})}{d_{\mathbb{R}, 1, S}(\mathrm{~L}, \mathrm{R})}\right)^{+}\right)^{2} \mathbf{1}_{\mathcal{A}_{i}^{c}}\right] \leq\left(\sum_{i \in Q_{*}} \mathbf{P}\left(\mathcal{A}_{i}^{c}\right)^{1 / \alpha}\right)\left(\max _{i \in Q_{*}} \mathbf{E}\left(\left(\left(\log \frac{d_{\mathbb{R}, 1, S}^{(i)}(\mathrm{L}, \mathrm{R})}{d_{\mathbb{R}, 1, S}(\mathrm{~L}, \mathrm{R})}\right)^{+}\right)^{2 \beta}\right)\right)^{1 / \beta} \\
\leq C K^{-c} \bar{\chi}_{S}^{b} \frac{\Theta_{\mathbb{B}(S)}^{\text {hard }}\left(p_{1}\right)}{\Theta_{\mathbb{B}(S)}^{\text {easy }}\left(p_{0}\right)} \leq C K^{-c} \bar{\chi}_{S}^{b+1} \leq C K^{-c} \bar{\chi}_{S}^{3},
\end{array}
$$

where in the penultimate inequality we recall Definition 4.3.

\subsubsection{Putting it all together: the proof of Lemma 5.7}

We are finally ready to prove Lemma 5.7, which will imply Theorem 5.1 as indicated at the end of Subsection 5.3 (on page 42).

Proof of Lemma 5.7. We have, using Lemma 5.8, Lemma 5.10, and Lemma 5.16 to bound the first three terms of (5.20), and Lemma 5.17 to bound the last term, that there are constants $C<\infty$ and $c>0$ so that as long as $K S \geq T_{0}$, we have

$\operatorname{Var}\left(\log d_{\mathbb{R}}(\mathrm{L}, \mathrm{R})\right)$

$$
\begin{aligned}
\leq & C \log K+C \mathrm{e}^{C \sqrt{\log K}}\left(\mathbf{E}_{j \in Q_{\circ}} d_{\left(3 \mathbb{E}_{i}\right)^{*}, \mathbb{A}_{j}, K^{-\gamma \theta_{0}, S}}(\text { around })^{3}\right)^{1 / 3}\left(\mathbf{E} d_{\mathbb{R}, 1, S}(\pi)^{-3}\right)^{1 / 3}+C K^{C} S^{-c} \\
& +2\left(\mathbf{E}\left[\max _{i \in Q_{*}} d_{\left(3 \mathbb{E}_{i}\right)^{*}, \mathbb{A}_{i}, K^{-\gamma \theta_{0}, S}}^{(i)}(\text { around })^{3}\right] \mathbf{E}\left[d_{\mathbb{R}, 1, S}(\mathrm{~L}, \mathrm{R})^{-3}\right] \mathbf{E}\left[\left(\sum_{i \in Q_{*}} \frac{\mathbf{1}_{\mathcal{A}_{i}} d_{\mathbb{R}^{*}, \mathbb{A}_{i}, 1, S}^{(i)}(\text { around })}{d_{\mathbb{R}, 1, S}(\mathrm{~L}, \mathrm{R})}\right)^{3}\right]\right)^{1 / 3} \\
& +C K^{-c} \bar{\chi}_{S}^{3} .
\end{aligned}
$$

Now for any $B \geq 3$ there is a constant $C$ so that

$$
\left(\mathbf{E}\left[\left(\sum_{i \in Q_{*}} \frac{\mathbf{1}_{\mathcal{A}_{i}} d_{\mathbb{R}^{*}, \mathbb{A}_{i}, 1, S}^{(\text {around })}}{d_{\mathbb{R}, 1, S}(\mathrm{~L}, \mathrm{R})}\right)^{3}\right]\right)^{1 / 3} \leq C K^{2 / B} \bar{\chi}_{K^{\eta} S}
$$

by Jensen's inequality and Lemma 5.14. Also, by Lemma 5.15, there is a $c>0$ so that, as long as $K^{\eta} S \geq T_{0}$,

$$
\left.\left(\mathbf{E}\left[\left(\max _{i \in Q_{*}} d_{\left(3 \mathbb{E}_{i}\right)^{*}, \mathbb{A}_{i}, K^{-\gamma \theta_{0}}}^{(i)} \text { (around }\right)\right)^{3}\right]\right)^{\frac{1}{3}},\left(\mathbf{E}\left[\left(\max _{i \in Q_{*}} d_{\left(3 \mathbb{E}_{i}\right)^{*}, \mathbb{A}_{i}, K^{-\gamma \theta_{0}}}(\text { around })\right)^{3}\right]\right)^{\frac{1}{3}} \leq C \bar{\chi}_{K^{\eta} S} K^{-c} \Theta_{\mathbb{B}(K S)}^{\text {easy }}\left(p_{0}\right) .
$$

Finally, we have

$$
\left(\mathbf{E}\left[d_{\mathbb{R}, 1, S}(\mathrm{~L}, \mathrm{R})^{-3}\right]\right)^{\frac{1}{3}}=\left(\mathbf{E}\left[d_{\mathbb{R}, 1, S}(\pi)^{-3}\right]\right)^{\frac{1}{3}} \leq C \bar{\chi}_{K S / 100} \Theta_{\mathbb{B}(K S)}^{\text {easy }}\left(p_{0}\right)^{-1}
$$

by Proposition 4.7 (applied with $Q=100$ ).

Plugging (5.81), (5.82), and (5.83) into the last term of (5.80), and (5.82) and (5.83) into the second term of (5.80), we obtain

$$
\begin{aligned}
\operatorname{Var}\left(\log d_{\mathbb{R}}(\mathrm{L}, \mathrm{R})\right) & \leq C \log K+C \mathrm{e}^{C \sqrt{\log K}} \bar{\chi}_{K^{\eta} S} K^{-c} \bar{\chi}_{K S / 100}+C K^{C} S^{-c}+C K^{2 / B-c} \bar{\chi}_{K S / 100} \bar{\chi}_{K^{\eta} S}^{2}+C K^{-c} \bar{\chi}_{S}^{3} \\
& \leq C \log K+C K^{-c / 2} \bar{\chi}_{K S / 100}^{3}+C K^{C} S^{-c},
\end{aligned}
$$


with the second equality valid so long as $B$ is chosen so large that $2 / B \leq c / 4$ and $K$ is large enough that $K \geq 100, K^{\eta-1} \geq 1 / 100$ and $\mathrm{e}^{C \sqrt{\log K}} \leq K^{c / 4}$.

\section{Subsequential scaling limits}

The variance bound proved in the previous section will allow us to show that the renormalized metric is "almost" Hölder continuous. (The "almost" is because the metric takes discrete values-this point is not important.) We recall the definition of $\Theta^{*}(S)$ in Corollary 5.2.

Theorem 6.1. There is a constant $c>0$ and a function $f: \mathbf{R}_{>0} \rightarrow \mathbf{R}_{>0}$ so that $\lim _{S \rightarrow \infty} f(S)=0$ and the following holds. For every $\varepsilon>0$, there is a constant $C=C(\varepsilon)<\infty$ so that for every $S \geq 0$, with probability at least $1-\varepsilon$, we have that for every $x, y \in \mathbb{B}(S)$,

$$
\frac{d_{\mathbb{B}(S)}(x, y)}{\Theta^{*}(S)} \leq C(\varepsilon)\left(\left|\frac{x-y}{S}\right|^{c} \vee f(S)\right) .
$$

Corollary 6.2. The family

$$
\left\{\frac{d_{\mathbb{B}(1), \delta}(\cdot, \cdot)}{\Theta^{*}\left(\delta^{-\frac{2}{\gamma^{2}+4}}\right)}\right\}_{\delta>0}
$$

is tight in the uniform topology on $[0,1]^{2}$.

Proof. This follows immediately from Theorem 6.1, Proposition 2.35, and Lemma A.4 (the last being a slight generalization of the Arzelà-Ascoli theorem).

We note that Corollary 6.2 immediately implies almost all of Theorem 1.1 by Proposition 2.25 and Prokhorov's theorem, except that the limiting objects may be pseudometrics rather than metrics. In this section we prove Theorem 6.1, and then in the next section we will prove that the limiting objects are in fact metrics. We will need a preliminary lemma, which allows us to deal with the "base case" of our diameter chaining argument by showing that it is unlikely that very small boxes will have large diameter.

Lemma 6.3. There is a $C<\infty$ and $a v_{0}>1$ so that if $v \in\left(1, v_{0}\right)$ then there is an $\alpha>0$ so that the following holds. Fix $S, K>0$ and let $\mathbb{R}=\mathbb{B}(S)$. Divide $\mathbb{R}^{\circ}$ into J-many $K^{-1} S \times K^{-1} S$ boxes labeled $\mathbb{C}_{1}, \ldots, \mathbb{C}_{J}$, with $J \leq C K^{2}$. Then for all $u>0$,

$$
\mathbf{P}\left(\max _{1 \leq j \leq J} \mu_{\mathbb{R}^{*}}\left(\mathbb{C}_{j}\right) \geq u\right) \leq C u^{-v} S^{\left(2+\gamma^{2} / 2\right) v} K^{-\alpha} .
$$

Proof. Define $\tilde{v}_{0}$ to be the $v_{0}$ from Proposition 2.11. Fix $v \in\left(1, \tilde{v}_{0}\right)$. For $1 \leq j \leq J$, define

$$
F_{j}=\max _{x \in \mathbb{C}_{j}^{\circ}} h_{\mathbb{R}^{*}: \mathbb{C}_{j}^{*}}(x)
$$

Then we note that $F_{j}$ and $\mu_{\mathbb{C}_{j}^{*}}\left(\mathbb{C}_{j}\right)$ are independent. Also, by (2.29), we have

$$
\mu_{\mathbb{R}^{*}}\left(\mathbb{C}_{j}\right) \leq \mathrm{e}^{\gamma F_{j}} \mu_{\mathbb{C}_{j}^{*}}\left(\mathbb{C}_{j}\right) .
$$

We recall from (2.29) and 2.9 that $F_{j}$ has expectation of order 1 and tails like those of a Gaussian with variance $\log K$. Therefore, we have

$$
\mathbf{E} \mathrm{e}^{\gamma v F_{j}} \leq C K^{\frac{1}{2} \gamma^{2} v^{2}}
$$


On the other hand, we have that $\mu_{\mathbb{C}_{j}^{*}}\left(\mathbb{C}_{j}\right) \stackrel{\text { law }}{=}(S / K)^{2+\gamma^{2} / 2} \mu_{\mathbb{B}^{*}}(\mathbb{B})$, where $\mathbb{B}=[0,1]^{2}$, so

$$
\mathbf{E} \mu_{\mathbb{C}_{j}^{*}}\left(\mathbb{C}_{j}\right)^{v}=(S / K)^{\left(2+\gamma^{2} / 2\right) v} \mathbf{E}\left[\mu_{\mathbb{B}^{*}}(\mathbb{B})^{v}\right]
$$

Therefore, by (6.2) and the independence of the two factors on its right-hand side, along with (6.3) and (6.4), we have

$$
\mathbf{E}\left[\mu_{\mathbb{R}^{*}}\left(\mathbb{C}_{j}\right)^{v}\right] \leq C S^{\left(2+\gamma^{2} / 2\right) v} K^{\frac{1}{2} \gamma^{2} v^{2}-\left(2+\gamma^{2} / 2\right) v} \mathbf{E}\left[\mu_{\mathbb{B}^{*}}(\mathbb{B})^{v}\right] \leq C S^{\left(2+\gamma^{2} / 2\right) v} K^{\frac{1}{2} \gamma^{2} v^{2}-\left(2+\gamma^{2} / 2\right) v}
$$

in which we note that $\mathbf{E} \mu_{\mathbb{B}^{*}}(\mathbb{B})^{v}$ is bounded above by a fixed absolute constant according to (2.13). Then we have

$$
\mathbf{E}\left[\max _{1 \leq j \leq J} \mu_{\mathbb{R}^{*}}\left(\mathbb{C}_{j}\right)^{v}\right] \leq J \mathbf{E}\left[\mu_{\mathbb{R}^{*}}\left(\mathbb{C}_{j}\right)^{v}\right] \leq C S^{\left(2+\gamma^{2} / 2\right) v} K^{\frac{1}{2} \gamma^{2} v^{2}-\left(2+\gamma^{2} / 2\right) v+2} .
$$

If we define $f(v)=\frac{1}{2} \gamma^{2} v^{2}-\left(2+\frac{1}{2} \gamma^{2}\right) v+2$, then $f(1)=0$ and $f^{\prime}(1)=\frac{1}{2} \gamma^{2}-2<0$, since $\gamma<2$. This implies that there is a $v_{0}>1$ such that so that $f(v)<0$ for $v \in\left(1, v_{0}\right)$, which means that if we further take $v_{0}<\tilde{v}_{0}$, then for each $v \in\left(1, v_{0}\right)$ we have an $\alpha>0$ so that

$$
\mathbf{E}\left[\max _{1 \leq j \leq J}\left[\mu_{\mathbb{R}^{*}}\left(\mathbb{C}_{j}\right)\right]^{\nu}\right] \leq C S^{\left(2+\gamma^{2} / 2\right) v} K^{-\alpha} .
$$

Inequality (6.1) then comes from Markov's inequality.

Proposition 6.4. There is a constant $c>0$ so that for every $\varepsilon>0$, there is a constant $C=C(\varepsilon)<\infty$ so that for every $S>0$ the following holds. Let $\mathbb{R}=\mathbb{B}(S)$. For integers $t \in \mathbf{Z}_{\geq 0}$ and $0 \leq i, j \leq 2^{t}-1$, define

$$
\mathbb{B}_{t ; i, j}= \begin{cases}\left(i 2^{-t} S, j 2^{-t+1} S\right)+\left[0,2^{-t} S\right] \times\left[0,2^{-t+1} S\right] & \text { if t is even } \\ \left(i 2^{-t+1} S, j 2^{-t} S\right)+\left[0,2^{-t+1} S\right] \times\left[0,2^{-t} S\right] & \text { if } t \text { is odd }\end{cases}
$$

Then

$$
\mathbf{P}\left(\bigcup_{t_{0}=0}^{\left\lfloor c \log _{2} S\right\rfloor}\left\{\max _{i, j=0}^{2^{t_{0}-1}} \sup _{x, y \in \mathbb{B}_{t_{0} ; i j}} \frac{d_{\mathbb{R}}(x, y)}{\Theta^{*}(S)} \geq C(\varepsilon) 2^{-c t_{0}}\right\}\right)<\varepsilon .
$$

Proof. Because the proof is somewhat technical and involves many parameters, we divide it into steps. The first step is to use a chaining argument to express the maximum in (6.5) in terms of a sum of hard crossing distances of smaller boxes at many scales. Then we define a budget for the hard crossing distance at each scale, and show that this budget is, with high probability, not exceeded at any scale.

Step 1: the chaining argument. By a chaining argument illustrated in Figure 6.1, we have, for any $t, t_{*} \in \mathbf{Z}_{\geq 0}$, that

$$
\begin{aligned}
\max _{i, j=0}^{2^{t}-1} \sup _{x, y \in \mathbb{B}_{t ; i, j}} d_{\mathbb{R}}(x, y) & \leq 2 \max _{i, j=0}^{2^{t_{*}}-1} \sup _{x, y \in \mathbb{B}_{t * i, j}} d_{\mathbb{R}^{*}, \mathbb{B}_{t * i, j}}(x, y)+2 \sum_{s=t}^{t_{*}} \max _{i, j=0}^{2^{s}-1} d_{\mathbb{R}^{*}, \mathbb{B}_{s ; i, j}} \text { (hard) } \\
& \leq C \max _{i, j=0}^{2^{t_{*}}-1} \mu_{\mathbb{R}^{*}}\left(\mathbb{B}_{t_{*} ; i, j}\right)+2 \sum_{s=t}^{t_{*}} \max _{i, j=0}^{2^{s}-1} d_{\mathbb{R}^{*}, \mathbb{B}_{s ; i, j}} \text { (hard). }
\end{aligned}
$$

(See [8, Proposition 6.7] for a similar construction described in more detail.) The second inequality in (6.6) is by the fact that all points in $\mathbb{B}_{t_{*} ; i, j}$ can be connected by some fixed number of balls inside $\mathbb{B}_{t_{*} ; i, j}^{\circ}$ with centers in $\mathbb{B}_{t_{*} ; i, j}$. 


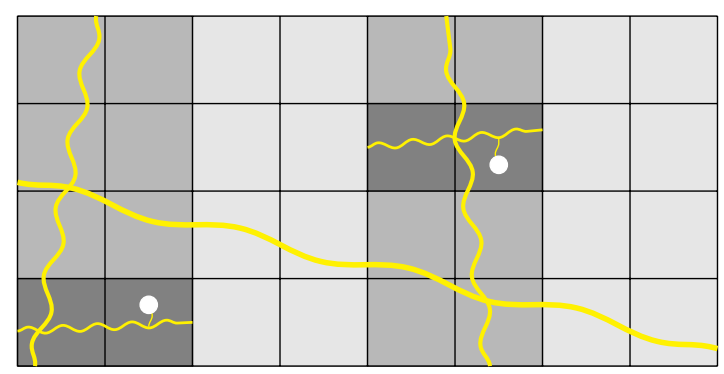

Figure 6.1: Connecting any two points with two hard crossings at each scale, plus two point-to-point paths within $\mathbb{B}_{t_{*} ; i, j}$ s. Any two points $x, y \in \mathbb{R}$ can be connected by a combination of a hard crossing in each of the $\mathbb{B}_{t ; i, j}$ s containing either $x$ or $y$, a connection from $x$ to a point on the hard crossing of the $\mathbb{B}_{t_{*} ; i, j}$ containing $x$, and a connection from $y$ to a point on the hard crossing of the $\mathbb{B}_{t_{*} ; i, j}$ containing $y$. This is because the hard crossing at each scale is guaranteed to intersect the hard crossings at the next smaller scale, and so all of the crossings can be joined into a crossing joining the two points.

We need to understand the coarse field on each of the $\mathbb{B}_{t ; i, j}$ s. To this end, we define

$$
F_{t ; i, j}=\max _{x \in \mathbb{B}_{t ; i, j}} h_{\mathbb{R}^{*}: \mathbb{B}_{t ; i, j}^{*}}(x), \quad F_{t}=\max _{i, j=0}^{2^{t}-1} F_{t ; i, j}
$$

Also define $\mathbb{S}_{s}=\mathbb{B}\left(2^{-(1-\eta) s}\right)$. By (2.41) of Proposition 2.38, (2.28), and Proposition 2.35, we have

$$
d_{\mathbb{R}^{*}, \mathbb{B}_{s ; i, j}}(\text { hard }) \leq d_{\mathbb{B}_{s ; i, j}, \mathrm{e}^{-\gamma F_{s}}}(\text { hard }) \leq \mathrm{e}^{\left(\gamma F_{s}-\gamma \theta_{0} s \log 2\right)^{+}} d_{\mathbb{B}_{s ; i, j}, 2^{-\gamma \theta_{0} s}}(\text { hard }) \stackrel{\operatorname{law}}{=} \mathrm{e}^{\left(\gamma F_{s}-\gamma \theta_{0} s \log 2\right)^{+}} d_{\mathbb{S}_{S}} \text { (hard). }
$$

By (6.6), (6.7), and a union bound, we have, for any choices of $B$ and $q_{t}\left(0 \leq t \leq t_{*}\right)$,

$$
\begin{aligned}
& \mathbf{P}\left(\bigcup_{t_{0}=0}^{t_{*}}\left\{\max _{i, j=0}^{2^{t_{0}}-1} \sup _{x, y \in \mathbb{B}_{t_{0} ; i, j}} d_{\mathbb{R}}(x, y) \geq 2 q_{t_{*}}+2 \sum_{t=t_{0}}^{t_{*}-1} 2^{B t^{2 / 3}} q_{t}\right\}\right) \\
& \quad \leq \sum_{t=1}^{t_{*}-1} \mathbf{P}\left[\mathrm{e}^{\gamma F_{t}-\gamma \theta_{0} t \log 2} \geq 2^{B t^{2 / 3}}\right]+\sum_{t=0}^{t_{*}-1} 4^{t} \mathbf{P}\left(d_{\mathbb{S}_{t}}(\text { hard }) \geq q_{t}\right)+\mathbf{P}\left(\max _{i, j=0}^{2^{t_{*}}-1} \mu_{\mathbb{R}^{*}}\left(\mathbb{B}_{t_{*} ; i, j}\right) \geq q_{t_{*}}\right) .
\end{aligned}
$$

Here, the $q_{t} \mathrm{~s}$ represent a "distance budget" that is available at each scale, so that the distance at each scale stays within the budget except on events whose probabilities we hope will be summable.

Step 2: defining the distance budget. To use (6.8), we have to define the budgets $q_{t}$, and then estimate the terms on the right-hand side. First, we define

$$
t_{1}=\left\lfloor(1-\eta)^{-1} \log _{2}\left(S / T_{0}\right)\right\rfloor
$$

so

$$
2^{-(1-\eta) t_{1}} S \geq T_{0}
$$

where $T_{0}$ is defined as in Proposition 4.4. Then, for $0 \leq t<t_{*}$, put

$$
q_{t}=\left(1+2^{A\left(t \wedge t_{1}\right)+D}\right) \Theta^{*}\left(2^{-(1-\eta) t} S\right),
$$

with constants $A$ and $D$ to be chosen later. By Proposition 4.8 and Corollary 5.2, we have

$$
\mathbf{P}\left(d_{\mathbb{S}_{t}}(\text { hard }) \geq q_{t}\right) \leq C \mathrm{e}^{-A^{8 / 7}\left(t \wedge t_{1}\right)^{8 / 7} / C-D^{8 / 7} / C} .
$$


On the other hand, by Corollary 5.2, Proposition 2.36, and Proposition 4.4 (recalling (6.10)) that there are constants $c>0$ and $C_{1}<\infty$ so that

$$
\Theta^{*}\left(2^{-(1-\eta) t} S\right) \leq \Theta^{*}\left(2^{-(1-\eta)\left(t \wedge t_{1}\right)} S\right) \leq C_{1} 2^{-c(1-\eta)\left(t \wedge t_{1}\right)} \Theta^{*}(S)
$$

Therefore, we have

$$
q_{t} \leq C_{1} 2^{(A-c(1-\eta))\left(t \wedge t_{1}\right)+D} \Theta^{*}(S)
$$

for all $0 \leq t<t_{*}$.

Also, by (2.9) of Corollary 2.8, we have

$$
\mathbf{P}\left[\mathrm{e}^{\gamma F_{t}-\gamma \theta_{0} t \log 2} \geq 2^{B t^{2 / 3}}\right] \leq C \exp \left\{-\frac{B^{2} t^{1 / 3}}{2 \gamma^{2}(\log 2)^{3}}\right\} .
$$

Substituting (6.11), (6.13), (6.12), and (6.1) from Lemma 6.3 into (6.8) yields, for any $v \in\left(1, v_{0}\right)$ (defining $v_{0}$ and also $\alpha$ as in Lemma 6.3), a constant $C$ so that

$$
\begin{gathered}
\mathbf{P}\left(\bigcup_{t_{0}=0}^{t_{*}-1}\left\{\max _{i, j=0}^{2^{t_{0}-1}} \sup _{x, y \in \mathbb{B}_{t_{0} ; i, j}} d_{\mathbb{R}}(x, y) \geq q_{t_{*}}+C_{1} \Theta^{*}(S) 2^{D} \sum_{t=t_{0}}^{t_{*}-1} 2^{B t^{2 / 3}+(A-c(1-\eta))\left(t \wedge t_{1}\right)}\right\}\right) \\
\leq C \sum_{t=0}^{t_{*}-1} \mathrm{e}^{-\frac{B^{2} t^{1 / 3}}{2 \gamma^{2}(\log 2)^{3}}}+C \mathrm{e}^{-D^{8 / 7}} \sum_{t=0}^{t_{*}-1} \mathrm{e}^{t \log 4-A^{8 / 7}\left(t \wedge t_{1}\right)^{8 / 7} / C}+C q_{t_{*}}^{-v} S^{\left(2+\gamma^{2} / 2\right) v} 2^{-t_{*} \alpha}
\end{gathered}
$$

Step 3: analyzing the right-hand side of (6.14). Now we fix $A=c(1-\eta) / 2$ and analyze the right-hand side of (6.14). To bound the third term, we take

$$
t_{*}=\left\lceil\frac{v}{\alpha}\left(2+\gamma^{2} / 2\right) \log _{2} S\right\rceil,
$$

so $S^{\left(2+\gamma^{2} / 2\right) v} 2^{-t_{*} \alpha} \leq 1$. We note that the first term of (6.14) can be bounded above by

$$
C \sum_{t=0}^{\infty} \mathrm{e}^{-\frac{B^{2} t^{1 / 3}}{2 \gamma^{2}(\log 2)^{3}}}
$$

which goes to 0 as $B \rightarrow \infty$. The second term of (6.14) can be bounded by writing

$$
\begin{aligned}
\sum_{t=0}^{t_{*}-1} \mathrm{e}^{t \log 4-A^{8 / 7}\left(t \wedge t_{1}\right)^{8 / 7} / C} & \leq \sum_{t=0}^{\infty} \mathrm{e}^{t \log 4-A^{8 / 7} t^{8 / 7} / C}+\mathrm{e}^{-A^{8 / 7} t_{1}^{8 / 7} / C} \sum_{t=t_{1}}^{t_{*}-1} 4^{t} \\
& \leq C\left(1+\exp \left\{-A^{8 / 7}\left((1-\eta)^{-1} \log _{2} \frac{S}{T_{0}}-1\right)^{8 / 7} / C+\left(\frac{v}{\alpha}\left(2+\gamma^{2} / 2\right) \log _{2} S\right) \log 4\right\}\right),
\end{aligned}
$$

using the values (6.9) and (6.15) of $t_{1}$ and $t_{*}$ fixed above. The right-hand side of (6.16) is also bounded as $S \rightarrow \infty$. Therefore, we have

$$
\lim _{\substack{B \rightarrow \infty \\ q_{t_{*}} \rightarrow \infty \\ \theta \rightarrow \infty}} \mathbf{P}\left(\bigcup_{t_{0}=0}^{t_{*}-1}\left\{\max _{i, j=0}^{2^{t_{0}-1}} \sup _{\substack{x, y \in \mathbb{B}_{t_{0} ; i, j}\\}} d_{\mathbb{R}}(x, y) \geq q_{t_{*}}+\theta \Theta^{*}(S) \sum_{t=t_{0}}^{t_{*}-1} 2^{B t^{2 / 3}-\frac{1}{2} c(1-\eta)\left(t \wedge t_{1}\right)}\right\}\right)=0
$$


uniformly in $S$. In particular, we can take $\theta=q_{t_{*}}=B$, and use Lemma 2.37 to fold these parameters into the sum, so the limit is simplified to

$$
\lim _{B \rightarrow \infty} \mathbf{P}\left(\bigcup_{t_{0}=0}^{t_{*}-1}\left\{\operatorname{lox}_{i, j=0}^{2_{0}-1} \sup _{x, y \in \mathbb{B}_{t_{0} ; i, j}} d_{\mathbb{R}}(x, y) \geq \Theta^{*}(S) \sum_{t=t_{0}}^{t_{*}-1} 2^{B t^{2 / 3}-\frac{1}{2} c(1-\eta)\left(t \wedge t_{1}\right)}\right\}\right)=0 .
$$

Step 4: analyzing the sum on the left-hand side of (6.17). The limit (6.17) yields a bound on the crossing distances, but we need to analyze the sum on the left-hand side to understand exactly what kind of bound. We have, for a constant $C(B)$ depending on $B$ but independent of $S$ and $t_{0}$, and a constant $c$ independent of $B, S, t_{0}$, that

$$
\begin{aligned}
\sum_{t=t_{0}}^{t_{*}-1} 2^{B t^{2 / 3}-\frac{1}{2} c(1-\eta)\left(t \wedge t_{1}\right)} & \leq C(B) \mathrm{e}^{-c_{1} t_{0}}+C 2^{-\frac{1}{2} c(1-\eta) t_{1}+(B+1) t_{*}^{2 / 3}} \\
& \leq C(B) \mathrm{e}^{-c_{1} t_{0}}+C 2^{-\frac{1}{2} c(1-\eta)\left((1-\eta)^{-1} \log _{2}\left(S / T_{0}\right)-1\right)+(B+1)\left(\frac{v}{\alpha}\left(2+\gamma^{2} / 2\right) \log _{2} S+1\right)^{2 / 3}},
\end{aligned}
$$

where the second inequality is by plugging in the definitions (6.9) and (6.15). Now we note that we can take $C(B)$ so that

$$
2^{-\frac{1}{2} c(1-\eta)\left((1-\eta)^{-1} \log _{2}\left(S / T_{0}\right)-1\right)+(B+1)\left(\frac{\nu}{\alpha}\left(2+\gamma^{2} / 2\right) \log _{2} S+1\right)^{2 / 3}} \leq C(B) S^{-c_{2}},
$$

where $c_{2}>0$ is a constant which does not depend on $B$. Also, as long as $t_{0} \leq \frac{c_{2}}{c_{1}} \log S$, we have $S^{-c_{2}} \leq \mathrm{e}^{-c_{1} t_{0}}$, so in this case by (6.18) we have

$$
\sum_{t=t_{0}}^{t_{*}-1} 2^{B t^{2 / 3}-\frac{1}{2} c(1-\eta)\left(t \wedge t_{1}\right)} \leq C(B) \mathrm{e}^{-c_{1} t_{0}}
$$

Plugging this into (6.17), we have

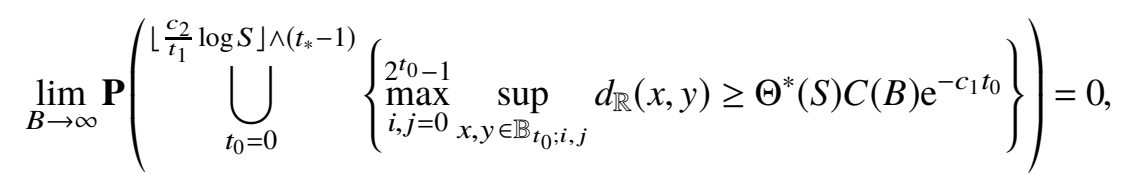

which is equivalent to (6.5).

Finally, we are ready to prove Theorem 6.1.

Proof of Theorem 6.1. By Proposition 6.4, for each $\varepsilon>0$ we have a constant $C=C(\varepsilon)$ so that, with probability $1-\varepsilon$, for each $0 \leq t_{0} \leq c \log _{2} S$,

$$
\sup _{\substack{x, y \in \mathbb{B}(S) \\|x-y| \leq 2^{-t_{0} S}}} \frac{d_{\mathbb{B}(S)}(x, y)}{\Theta^{*}(S)} \leq 2 C(\varepsilon) 2^{-c t_{0}} .
$$

Therefore,

$$
\sup _{x, y \in \mathbb{B}(S)} \frac{d_{\mathbb{R}}(x, y) / \Theta^{*}(S)}{\left|\frac{x-y}{S}\right| \vee S^{-c}} \leq 2 C(\varepsilon)
$$

with probability $1-\varepsilon$, so the theorem is proved. 


\section{Bi-Hölder continuity of the limiting metrics}

Up to now, we have established the existence of a subsequential limiting function for our sequence of approximating metrics. It is of course clear that any limiting function must satisfy positivity, symmetry, and the triangle inequality, qualifying it for the title of "pseudo-metric." However, it is not a priori clear that a limiting pseudometric must be a true metric — that is, that it is positive definite. That is what we will prove in this section. In fact, we will prove the stronger statement that the Euclidean metric must be Hölder continuous with respect to any limiting LGD metric. The following proposition gives a uniform upper bound on the moments of the Hölder coefficient, from which the Hölder continuity of the Euclidean metric with respect to any subsequential limit of the LGD metric follows by Fatou's lemma.

Proposition 7.1. For every $B \in(1, \infty)$, we have an $\alpha_{0}>0$ so that for any $\alpha \in\left(0, \alpha_{0}\right)$, there is a constant $C<\infty$ so that for any $\delta>0$, if we define $\mathbb{B}=\mathbb{B}(1)$, then

$$
\mathbf{E}\left[\left(\max _{x, y \in \mathbb{B}} \frac{|x-y|}{\left[d_{\mathbb{B}, \delta}(x, y) / \Theta^{*}\left(\delta^{-\frac{2}{\gamma^{2}+4}}\right)\right]^{\alpha}}\right)^{B}\right] \leq C .
$$

Proof. Note that

$$
\begin{aligned}
\max _{x, y \in \mathbb{B}} \frac{|x-y|}{\left[d_{\mathbb{B}, \delta}(x, y) / \Theta^{*}\left(\delta^{-\frac{2}{\gamma^{2}+4}}\right)\right]^{\alpha}} & =\max _{n \in \mathbf{N}} \max _{\substack{x, y \in \mathbb{B} \\
|x-y| \geq 2^{-n}}} \frac{|x-y|}{\left[d_{\mathbb{B}, \delta}(x, y) / \Theta^{*}\left(\delta^{-\frac{2}{\gamma^{2}+4}}\right)\right]^{\alpha}} \\
& \leq 2 \max _{n \in \mathbf{N}}\left(2^{-n}\left[\frac{d_{\mathbb{B}, \delta}\left(\min ; 2^{-n}\right)}{\Theta^{*}\left(\delta^{-\frac{2}{\gamma^{2}+4}}\right)}\right]^{-\alpha}\right) .
\end{aligned}
$$

By Proposition 4.7 and Theorem 5.1, we have for every $A<\infty$ a constant $C<\infty$ so that, for each $a \in(0,1)$, we have

$$
\mathbf{E}\left[d_{\mathbb{B}}(\min ; a)^{-A}\right] \leq C\left(1+a^{-C}\right)\left[\Theta^{*}\left(\delta^{-\frac{2}{\gamma^{2}+4}}\right)\right]^{-A} .
$$

This implies that

$$
\mathbf{E}\left[\max _{n \in \mathbf{N}}\left(\frac{d_{\mathbb{B}}\left(\min ; 2^{-n}\right) / \Theta^{*}\left(\delta^{-\frac{2}{\gamma^{2}+4}}\right)}{2^{-n / \alpha}}\right)^{-A}\right] \leq \sum_{n=0}^{\infty} \mathbf{E}\left[\left(\frac{d_{\mathbb{B}}\left(\min ; 2^{-n}\right) / \Theta^{*}\left(\delta^{-\frac{2}{\gamma^{2}+4}}\right)}{2^{-n / \alpha}}\right)^{-A}\right] \leq C \sum_{n=0}^{\infty} \frac{\left(1+2^{C n}\right)}{2^{A n / \alpha}},
$$

where the constant $C$ still depends on $A$. As long as $\alpha$ is chosen small enough so that $A / \alpha \geq C$, the last sum is finite. In particular, choose $\alpha$ so small that $A / \alpha \geq B$. Then we have

$$
\begin{aligned}
\mathbf{E}\left[\max _{n \in \mathbf{N}}\left(\frac{d_{\mathbb{B}}\left(\min ; 2^{-n}\right) / \Theta^{*}\left(\delta^{-\frac{2}{\gamma^{2}+4}}\right)}{\left[d_{\mathbb{B}, \delta}(x, y) / \Theta^{*}\left(\delta^{-\frac{2}{\gamma^{2}+4}}\right)\right]^{\alpha}}\right)^{A / \alpha}\right] & \leq \mathbf{E}\left[\left(2 \max _{n \in \mathbf{N}} 2^{-n}\left[\frac{d_{\mathbb{B}, \delta}\left(\min ; 2^{-n}\right)}{\Theta^{*}\left(\delta^{-\frac{2}{\gamma^{2}+4}}\right)}\right]^{-\alpha}\right)^{A / \alpha}\right] \\
& =2^{A / \alpha} \mathbf{E}\left[\max _{n \in \mathbf{N}}\left(\frac{d_{\mathbb{B}, \delta}\left(\min ; 2^{-n}\right) / \Theta^{*}\left(\delta^{-\frac{2}{\gamma^{2}+4}}\right)}{2^{-n / \alpha}}\right)^{-A}\right] \leq C,
\end{aligned}
$$

where the first inequality is by (7.2) and the second is by (7.3). Then (7.1) follows by Jensen's inequality. 


\section{A Technical lemmas}

Lemma A.1. Let $X$ be a positive random variable and let $\Theta_{X}$ be its quantile function. For any $p, q \in(0,1)$, we have

$$
\left.\Theta_{X}(q) \leq \exp \left\{\sqrt{\operatorname{Var}(\log X)}\left((1-q)^{-1 / 2}+p^{-1 / 2}\right)\right)\right\} \Theta_{X}(p) .
$$

Proof. Define $\mu=\mathbf{E} \log X$. We have, for $a \geq \mathrm{e}^{\mu}$,

$$
1-F_{X}(a)=\mathbf{P}(X \geq a)=\mathbf{P}(\log X \geq \log a)=\mathbf{P}(\log X-\mu \geq \log a-\mu) \leq \frac{\operatorname{Var}(\log X)}{(\log a-\mu))^{2}} .
$$

This means that $\Theta_{X}\left(1-\frac{\operatorname{Var}(\log X)}{(\log a-\mu)^{2}}\right) \leq a$. Now if $a=\exp \left\{\sqrt{\frac{\operatorname{Var}(\log X)}{1-p}}+\mu\right\}$, then $a \geq \exp \{\mathbf{E} \log X\}$ and $1-$ $\frac{\operatorname{Var}(\log X)}{(\log a-\mu)^{2}}=p$, so

$$
\Theta_{X}(p) \leq \exp \left\{\sqrt{\frac{\operatorname{Var}(\log X)}{1-p}}+\mu\right\} .
$$

Similarly, we have, if $a \leq \exp \{\mathbf{E} \log X\}$,

$$
F_{X}(a)=\mathbf{P}(X \leq a)=\mathbf{P}(\log X \leq \log a) \leq \mathbf{P}(\log X-\mu \leq \log a-\mu) \leq \frac{\operatorname{Var}(\log X)}{(\log a-\mu)^{2}}
$$

Therefore, $a \leq \Theta_{X}\left(\frac{\operatorname{Var}(\log X)}{(\log a-\mu)^{2}}\right)$. Now if $a=\exp \left\{-\sqrt{\frac{\operatorname{Var}(\log X)}{p}}+\mu\right\}$, then $a \leq \exp \{\mathbf{E} \log X\}$ and $\frac{\operatorname{Var}(\log X)}{(\log a-\mu)^{2}}=p$, so

$$
\Theta_{X}(p) \geq \exp \left\{-\sqrt{\frac{\operatorname{Var}(\log X)}{p}}+\mu\right\} .
$$

Therefore, for any $p, q \in(0,1)$, we have

$$
\begin{aligned}
\Theta_{X}(q) & \left.\leq \exp \left\{\sqrt{\frac{\operatorname{Var}(\log X)}{1-q}}+\mu\right\} \leq \exp \left\{\sqrt{\operatorname{Var}(\log X)}\left((1-q)^{-1 / 2}+p^{-1 / 2}\right)\right)\right\} \exp \left\{-\sqrt{\frac{\operatorname{Var}(\log X)}{p}}+\mu\right\} \\
& \left.\leq \exp \left\{\sqrt{\operatorname{Var}(\log X)}\left((1-q)^{-1 / 2}+p^{-1 / 2}\right)\right)\right\} \Theta_{X}(p) .
\end{aligned}
$$

Lemma A.2. Suppose that $p<\frac{1}{2}$ and $X_{1}, \ldots, X_{N}$ are iid Bernoulli $(p)$ random variables. Let $S_{N}=\sum_{i=1}^{N} X_{i}$. Then

$$
\mathbf{P}\left[S_{N} / N \geq 1 / 2\right] \leq(8 p)^{N / 2}
$$

Proof. We have

$$
\mathbf{P}\left[S_{N} / N \geq 1 / 2\right]=\mathbf{P}\left[\mathrm{e}^{\lambda S_{N}} \geq \mathrm{e}^{\lambda N / 2}\right] \leq \frac{\left(\mathbf{E e}^{\lambda X_{i}}\right)^{N}}{\mathrm{e}^{\lambda N / 2}}=\left(\frac{p \mathrm{e}^{\lambda}+1-p}{\mathrm{e}^{\lambda / 2}}\right)^{N}
$$

Putting $\lambda=\log \frac{1-p}{p}$, we obtain

$$
\mathbf{P}\left[S_{N} / N \geq 1 / 2\right] \leq\left(2(1-p)\left(\frac{1-p}{p}\right)^{-\frac{1}{2}}\right)^{N} \leq(8 p)^{N / 2}
$$


Lemma A.3. Suppose that $\mathbb{B}$ is a rectangle and $\operatorname{dist}_{\mathrm{E}}(x, \partial \mathbb{B}) \geq c \operatorname{diam}_{\mathrm{E}}(\mathbb{B})$ for some constant $c$. Then there is a constant $c$, depending on $C$, so that

$$
\left|\nabla_{y} p_{t}^{\mathbb{B}}(x, y)\right| \leq C \frac{|x-y|}{t^{2}} \mathrm{e}^{-\frac{|x-y|^{2}}{2 t}} .
$$

Proof. This proof essentially appeared before in [34, Appendix 1] and was refined in [12], but as we need a somewhat different statement, we present the proof in full. We have

$$
p_{t}^{\mathbb{B}}(x, y)=\frac{\mathrm{e}^{-\frac{|x-y|^{2}}{2 t}}}{2 \pi t} q_{t}^{\mathbb{B}}(x, y),
$$

where

$$
q_{t}^{\mathbb{B}}(x, y)=\mathbf{P}\left(B_{s}-\frac{s}{t} B_{t}+x+\frac{s}{t}(y-x) \in \mathbb{B} \text { for all } s \leq t\right) .
$$

Therefore, we have

$$
\nabla_{y} p_{t}^{\mathbb{B}}(x, y)=-(x-y) \frac{\mathrm{e}^{-\frac{|x-y|^{2}}{2 t}}}{4 \pi t^{2}} q_{t}^{\mathbb{B}}(x, y)+\frac{\mathrm{e}^{-\frac{|x-y|^{2}}{2 t}}}{2 \pi t} \nabla_{y} q_{t}^{\mathbb{B}}(x, y) .
$$

Fix $y_{1}, y_{2} \in \mathbb{R}$. Let $z^{(1)}$ be the $x$-coordinate of the right edge of $\mathbb{B}$. Let $E_{i}$ be the event

$$
\left\{\max _{s \leq t}\left(B_{s}-\frac{s}{t} B_{t}+x+\frac{s}{t}\left(y_{i}-x\right)\right)^{(1)}>z^{(1)}\right\}
$$

where the superscript (1) means to consider the $x$-coordinate. We note that

$$
\left|\left(B_{s}-\frac{s}{t} B_{t}+x+\frac{s}{t}\left(y_{1}-x\right)\right)^{(1)}-\left(B_{s}-\frac{s}{t} B_{t}+x+\frac{s}{t}\left(y_{2}-x\right)\right)^{(1)}\right| \leq\left|y_{1}-y_{2}\right| .
$$

Therefore, if $E_{1}$ occurs but $E_{2}$ does not, then we must have that

$$
\max _{s \leq t}\left(B_{s}-\frac{s}{t} B_{t}+x+\frac{s}{t}\left(y_{1}-x\right)\right)^{(1)} \in\left[z^{(1)}, z^{(1)}+\left|y_{1}-y_{2}\right|\right] .
$$

We have that

$$
\begin{aligned}
\mathbf{P} & \left(\max _{s \leq t}\left(B_{s}-\frac{s}{t} B_{t}+x+\frac{s}{t}\left(y_{1}-x\right)\right)^{(1)} \in\left[z^{(1)}, z^{(1)}+\left(y_{1}-y_{2}\right)^{(1)}\right]\right) \\
= & \mathbf{P}\left(\max _{s \leq t}\left(B_{s}-\frac{s}{t} B_{t}+x+\frac{s}{t}\left(y_{1}-x\right)\right)^{(1)} \geq z^{(1)}\right) \\
& \quad-\mathbf{P}\left(\max _{s \leq t}\left(B_{s}-\frac{s}{t} B_{t}+x+\frac{s}{t}\left(y_{1}-x\right)\right)^{(1)} \geq z^{(1)}+\left(y_{1}-y_{2}\right)^{(1)}\right) .
\end{aligned}
$$

By the reflection principle, we have that

$$
\begin{aligned}
\mathbf{P}\left(\max _{s \leq t}\left(B_{s}-\frac{s}{t} B_{t}+x+\frac{s}{t}\left(y_{1}-x\right)\right)^{(1)} \geq z^{(1)}\right) & =\frac{1}{2} \mathrm{e}^{-\frac{\left(z^{(1)}+y_{1}^{(1)}-x\right)^{2}+\left(y_{1}^{(1)}-x\right)^{2}}{2 t}} \\
\mathbf{P}\left(\max _{s \leq t}\left(B_{s}-\frac{s}{t} B_{t}+x+\frac{s}{t}\left(y_{1}-x\right)\right)^{(1)} \geq z^{(1)}+\left(y_{1}-y_{2}\right)^{(1)}\right) & =\frac{1}{2} \mathrm{e}^{-\frac{\left(z^{(1)}+2 y_{1}^{(1)}-y^{(2)}-x\right)^{2}+\left(y_{1}^{(1)}-x\right)^{2}}{2 t}},
\end{aligned}
$$

so

$$
\mathbf{P}\left(E_{1} \backslash E_{2}\right) \leq \frac{1}{2}\left(\mathrm{e}^{-\frac{\left(z^{(1)}+y_{1}^{(1)}-x\right)^{2}+\left(y_{1}^{(1)}-x\right)^{2}}{2 t}}-\mathrm{e}^{-\frac{\left(z^{(1)}+2 y_{1}^{(1)}-y^{(2)}-x\right)^{2}+\left(y_{1}^{(1)}-x\right)^{2}}{2 t}}\right)
$$


Now we have

$$
\begin{aligned}
\lim _{y_{2}^{(1)} \rightarrow y_{1}^{(1)}} & \frac{1}{2\left(y_{2}^{(1)}-y_{1}^{(1)}\right)}\left(\mathrm{e}^{-\frac{\left(z^{(1)}+y_{1}^{(1)}-x\right)^{2}+\left(y_{1}^{(1)}-x\right)^{2}}{2 t}}-\mathrm{e}^{-\frac{\left(z^{(1)}+2 y_{1}^{(1)}-y^{(2)}-x\right)^{2}+\left(y_{1}^{(1)}-x\right)^{2}}{2 t}}\right) \\
= & \frac{\left(z^{(1)}+y_{1}^{(1)}-x\right)}{2 t} \mathrm{e}^{-\frac{\left(z^{(1)}+y_{1}^{(1)}-x\right)^{2}+\left(y_{1}^{(1)}-x\right)^{2}}{2 t}} .
\end{aligned}
$$

This implies that $\left|\nabla_{y} q_{t}^{\mathbb{B}}\left(x, y_{1}\right)\right| \leq C \frac{\operatorname{diam}_{E}(\mathbb{R})}{2 t} \exp \left\{-\frac{\operatorname{diam}_{E}(\mathbb{R})^{2}}{C t}\right\}$, and plugging into (A.1) we obtain

$$
\left|\nabla_{y} p_{t}^{\mathbb{B}}(x, y)\right| \leq \frac{|x-y|}{4 \pi t^{2}} \mathrm{e}^{-\frac{|x-y|^{2}}{2 t}}+C \frac{\mathrm{e}^{-\frac{|x-y|^{2}}{2 t}}}{4 \pi t^{2}} \operatorname{diam}_{\mathrm{E}}(\mathbb{R}) \exp \left\{-\frac{\operatorname{diam}_{\mathrm{E}}(\mathbb{R})^{2}}{C t}\right\} \leq C \frac{|x-y|}{t^{2}} \mathrm{e}^{-\frac{|x-y|^{2}}{2 t}}
$$

Lemma A.4. Let $\mathbb{X}$ be a rectangular subset of $\mathbf{R}^{d}$ and suppose that we have a family of functions $\left\{f_{n}: \mathbb{X} \rightarrow\right.$ $\mathbf{R}$ \} so that, for some constant $C$, we have

$$
\left|f_{n}(x)\right| \leq C
$$

and

$$
\left|f_{n}(x)-f_{n}(y)\right| \leq C\left(|x-y|^{\alpha} \vee \beta_{n}\right)
$$

for some $\alpha>0$ and some sequence $\beta_{n} \rightarrow 0$. Then the family $\left\{f_{n}: \mathbb{X} \rightarrow \mathbf{R}\right\}$ is precompact in the uniform topology.

Proof. Extend $f_{n}$ to an open subset $\mathbb{X}^{\prime}$ of $\mathbf{R}^{d}$ containing $\mathbb{X}$ by reflecting it across the boundaries; note that this can be done in such a way that (A.3) still holds, perhaps with a larger constant $C$. Let $\rho: \mathbf{R}^{d} \rightarrow \mathbf{R}$ be a smooth positive function such that $\operatorname{supp} \rho \subset B(0,1)$ and define $\rho_{\varepsilon}(x)=\varepsilon^{-d} \rho\left(\varepsilon^{-1} x\right)$. Let $*$ denote convolution. We have that

$$
\begin{aligned}
\left|\rho_{\varepsilon} * f_{n}(x)-f_{n}(x)\right| & =\left|\int \rho_{\varepsilon}(y) f_{n}(x-y) \mathrm{d} y-f_{n}(x)\right| \leq \int \rho_{\varepsilon}(y)\left|f_{n}(x-y)-f_{n}(x)\right| \mathrm{d} y \\
& \leq C \int \rho_{\varepsilon}(y)\left(|y|^{\alpha} \vee \beta_{n}\right) \mathrm{d} x \leq C\left(\varepsilon^{\alpha} \vee \beta_{n}\right) .
\end{aligned}
$$

Moreover, if $|x-z|^{\alpha} \geq \beta_{n}$, then we have

$$
\left|\rho_{\varepsilon} * f_{n}(x)-\rho_{\varepsilon} * f_{n}(z)\right|=\left|\int\left[f_{n}(x-y)-f_{n}(z-y)\right] \rho_{\varepsilon}(y) \mathrm{d} y\right| \leq C|x-z|^{\alpha},
$$

while if $|x-z|^{\alpha} \leq \beta_{n}$, then we have, if $\varepsilon=\beta_{n}$, that

$$
\begin{aligned}
\left|\rho_{\varepsilon} * f_{n}(x)-\rho_{\varepsilon} * f_{n}(z)\right| & =\int f_{n}(y)\left[\rho_{\varepsilon}(x-y)-\rho_{\varepsilon}(z-y)\right] \mathrm{d} y=\int\left[f_{n}(y)-f_{n}(x)\right] \cdot\left[\rho_{\varepsilon}(x-y)-\rho_{\varepsilon}(z-y)\right] \mathrm{d} y \\
& \leq C \beta_{n} \int\left|\rho_{\varepsilon}(x-y)-\rho_{\varepsilon}(z-y)\right| \mathrm{d} y \leq C\|\rho\|_{C^{1}} \beta_{n} \varepsilon^{-1}|x-z|=C|x-z| .
\end{aligned}
$$

Together, (A.5) and (A.6) imply the family $\left\{\rho_{\beta_{n}} * f_{n}\right\}$ is equicontinuous; since the family is evidently bounded by (A.2), the Arzelà-Ascoli theorem implies that there is a continuous function $f$ and a subsequence $\left(n_{k}\right)$ so that

$$
\lim _{k \rightarrow \infty} \rho_{\beta_{n_{k}}} * f_{n_{k}}=f
$$

uniformly. On the other hand, (A.4) implies that

$$
\lim _{k \rightarrow \infty}\left|\rho_{\beta_{n}} * f_{n}-f_{n}\right|=0
$$

uniformly. Combining (A.7) and (A.8) implies that $\lim _{k \rightarrow \infty} f_{n}=f$, which completes the proof. 


\section{References}

[1] R. J. Adler. An introduction to continuity, extrema, and related topics for general Gaussian processes, volume 12 of Institute of Mathematical Statistics Lecture Notes-Monograph Series. Institute of Mathematical Statistics, Hayward, CA, 1990.

[2] R. Allez, R. Rhodes, and V. Vargas. Lognormal «-scale invariant random measures. Probab. Theory Related Fields, 155(3-4):751-788, 2013.

[3] O. Angel. Growth and percolation on the uniform infinite planar triangulation. Geom. Funct. Anal., 13(5):935-974, 2003.

[4] J. Aru, E. Powell, and A. Sepúlveda. Critical Liouville measure as a limit of subcritical measures. Electron. Commun. Probab., 24:Paper No. 18, 16, 2019.

[5] N. Berestycki. Introduction to the Gaussian free field and Liouville quantum gravity. Lecture notes available on the webpage of the author, July 2016. URL http://www.statslab.cam.ac.uk/ beresty/Articles/oxford4.pdf.

[6] N. Berestycki. An elementary approach to Gaussian multiplicative chaos. Electron. Commun. Probab., 22:Paper No. 27, 12, 2017.

[7] M. Biskup. Extrema of the two-dimensional discrete Gaussian free field, arXiv: 1712.09972v4.

[8] J. Ding and A. Dunlap. Liouville first-passage percolation: subsequential scaling limits at high temperature. Ann. Probab., 47(2):690-742, 2019.

[9] J. Ding and S. Goswami. Upper bounds on Liouville first passage percolation and Watabiki's prediction. Comm. Pure Appl. Math., to appear, arXiv: 1610.09998v4.

[10] J. Ding and E. Gwynne. The fractal dimension of Liouville quantum gravity: universality, monotonicity, and bounds. Comm. Math. Phys., to appear, arXiv: 1807.01072v3.

[11] J. Ding, O. Zeitouni, and F. Zhang. On the Liouville heat kernel for $k$-coarse MBRW. Electron. J. Probab., 23:Paper No. 62, 20, 2018. URL https://doi.org/10.1214/18-EJP189.

[12] J. Ding, O. Zeitouni, and F. Zhang. Heat kernel for Liouville Brownian motion and Liouville graph distance. Comm. Math. Phys., to appear, arXiv: 1807.00422v1.

[13] J. Ding and F. Zhang. Non-universality for first passage percolation on the exponential of log-correlated Gaussian fields. Probab. Theory Related Fields, 171(3-4):1157-1188, 2018.

[14] J. Ding and F. Zhang. Liouville first passage percolation: geodesic length exponent is strictly larger than 1 at high temperatures. Probab. Theory Related Fields, 174(1-2):335-367, 2019.

[15] J. Dubédat and H. Falconet. Liouville metric of star-scale invariant fields: tails and Weyl scaling. Probab. Theory Related Fields, to appear, arXiv: 1809.02607v1.

[16] B. Duplantier, J. Miller, and S. Sheffield. Liouville quantum gravity as a mating of trees, arXiv: $1409.7055 \mathrm{v} 3$.

[17] B. Duplantier and S. Sheffield. Liouville quantum gravity and KPZ. Invent. Math., 185(2):333-393, 2011. 
[18] X. Fernique. Regularité des trajectoires des fonctions aléatoires gaussiennes. In École d'Été de Probabilités de Saint-Flour, IV-1974, volume 480 of Lecture Notes in Math., page 1-96. Springer-Verlag Berlin Heidelberg, 1975.

[19] E. Gwynne, N. Holden, and X. Sun. A mating-of-trees approach for graph distances in random planar maps, arXiv: 1711.00723v2.

[20] E. Gwynne, N. Holden, and X. Sun. A distance exponent for Liouville quantum gravity. Probab. Theory Related Fields, 173(3-4):931-997, 2019.

[21] J.-P. Kahane. Sur le chaos multiplicatif. Ann. Sci. Math. Québec, 9(2):105-150, 1985.

[22] J.-F. Le Gall. Geodesics in large planar maps and in the Brownian map. Acta Math., 205(2):287-360, 2010.

[23] J.-F. Le Gall. Uniqueness and universality of the Brownian map. Ann. Probab., 41(4):2880-2960, 2013.

[24] M. Ledoux. The concentration of measure phenomenon, volume 89 of Math. Surveys Monogr. American Mathematical Society, Providence, RI, 2001.

[25] T. Madaule. Maximum of a log-correlated Gaussian field. Ann. Inst. Henri Poincaré Probab. Stat., 51(4):1369-1431, 2015.

[26] P. Maillard, R. Rhodes, V. Vargas, and O. Zeitouni. Liouville heat kernel: regularity and bounds. Ann. Inst. Henri Poincaré Probab. Stat., 52(3):1281-1320, 2016.

[27] G. Miermont. The Brownian map is the scaling limit of uniform random plane quadrangulations. Acta Math., 210(2):319-401, 2013.

[28] J. Miller and S. Sheffield. Liouville quantum gravity and the Brownian map II: geodesics and continuity of the embedding, arXiv: 1605.03563v2.

[29] J. Miller and S. Sheffield. Liouville quantum gravity and the Brownian map III: the conformal structure is determined, arXiv: 1608.05391v2.

[30] J. Miller and S. Sheffield. Quantum Loewner evolution. Duke Math. J., 165(17):3241-3378, 2016.

[31] J. Miller and S. Sheffield. Liouville quantum gravity and the Brownian map I: The QLE(8/3,0) metric. Invent. Math., to appear, arXiv: 1507.00719v3.

[32] G. M. Molchan. Scaling exponents and multifractal dimensions for independent random cascades. Comm. Math. Phys., 179(3):681-702, 1996.

[33] R. Rhodes and V. Vargas. KPZ formula for log-infinitely divisible multifractal random measures. ESAIM. Probability and Statistics, 15:358-371, 2011.

[34] R. Rhodes and V. Vargas. Gaussian multiplicative chaos and applications: a review. Probab. Surv., 11:315-392, 2014.

[35] R. Rhodes and V. Vargas. The tail expansion of Gaussian multiplicative chaos and the Liouville reflection coefficient. Ann. Probab., to appear, arXiv: 1710.02096v3.

[36] L. Russo. A note on percolation. Z. Wahrscheinlichkeitstheorie und Verw. Gebiete, 43(1):39-48, 1978. 
[37] L. Russo. On the critical percolation probabilities. Z. Wahrscheinlichkeitstheorie und Verw. Gebiete, 56(2):229-237, 1981.

[38] P. D. Seymour and D. J. A. Welsh. Percolation probabilities on the square lattice. Ann. Discrete Math., 3:227-245, 1978. Advances in graph theory (Cambridge Combinatorial Conf., Trinity College, Cambridge, 1977).

[39] A. Shamov. On Gaussian multiplicative chaos. J. Funct. Anal., 270(9):3224-3261, 2016.

[40] S. Sheffield. Gaussian free fields for mathematicians. Probab. Theory Related Fields, 139(34):521-541, 2007.

[41] V. Tassion. Crossing probabilities for Voronoi percolation. Ann. Probab., 44(5):3385-3398, 2016. 